MEASURING THE DYNAMIC ENERGY EFFICIENCY OF FPGAS OVER PROCESSORS

by

\author{
Muhammad Umair Zafar, \\ Bachelor of Electrical Engineering(Electronics), \\ Bahria University, Islamabad, July 2010
}

A thesis presented to

Ryerson University

in partial fulfillment of the

requirements for the degree of

Master of Applied Science

in the Program of

Electrical and Computer Engineering

Toronto, Ontario, Canada, 2016

(C) Muhammad Umair Zafar, 2016 


\section{AUTHOR'S DECLARATION FOR ELECTRONIC SUBMISSION OF A THESIS}

I hereby declare that I am the sole author of this thesis. This is a true copy of the thesis, including any required final revisions, as accepted by my examiners.

I authorize Ryerson University to lend this thesis to other institutions or individuals for the purpose of scholarly research.

I further authorize Ryerson University to reproduce this thesis by photocopying or by other means, in total or in part, at the request of other institutions or individuals for the purpose of scholarly research.

I understand that my thesis may be made electronically available to the public. 


\title{
Measuring the Dynamic Energy Efficiency of FPGAs over Processors
}

\author{
Muhammad Umair Zafar \\ Master of Applied Science \\ Electrical and Computer Engineering \\ Ryerson University \\ 2016
}

\begin{abstract}
This work investigates the dynamic energy efficiency of the parallel execution model of an FPGA and the sequential execution model of a processor, for latency-insensitive applications. We create the temporal implementations (sequential instructions) of the MCNC benchmarks to be executed on a processor that employs a 4LUT as its functional unit. This processor is $\sim 716$ times inefficient for dynamic energy than a 4LUT FPGA, mainly due to the large amount of memory (instruction/data) that is required to encode the 4LUT based instructions. The size of the memory (instruction/data) can be reduced by increasing the data-path width and the logic complexity of the ASIC-based functional units of the processor. Particularly, at 64-bit data-path width and when the (instruction/data) memory sizes are reduced to less than $\sim 9 \%$ of their corresponding 4LUT-based instructions, the processor with ASIC-based complex functional unit can achieve higher dynamic energy efficiency than the FPGA for MCNC benchmarks.
\end{abstract}




\section{Acknowledgements}

First of all, I would like to say thanks to my supervisor Dr. Andy Ye, for his support and supervision during this work. I sincerely appreciate the time and efforts he invested in mentoring me.

I would like to say thanks to my friends and lab partners. Also I would like to thank Ryerson University for providing me an opportunity and good environment to perform this research.

I would like to say thanks to my family, without their support this work would have never been possible. 


\section{Dedication(s)}

To my wife Safoora 


\section{Contents}

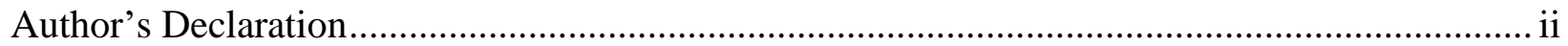

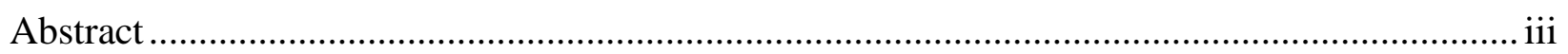

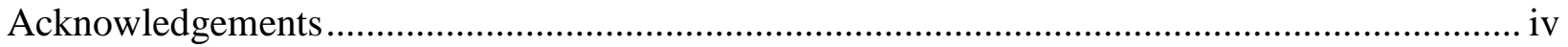

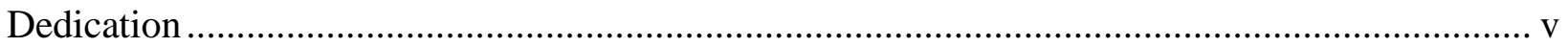

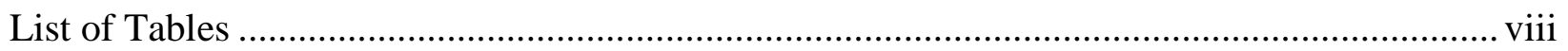

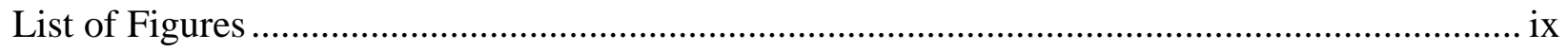

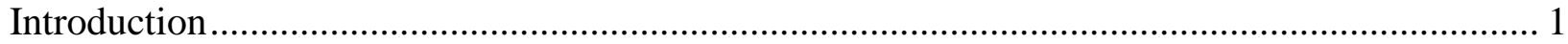

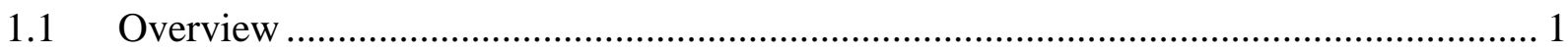

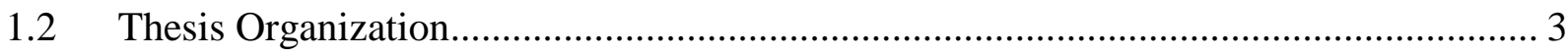

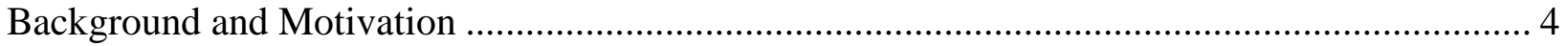

2.1 Field Programmable Gate Arrays ......................................................................... 4

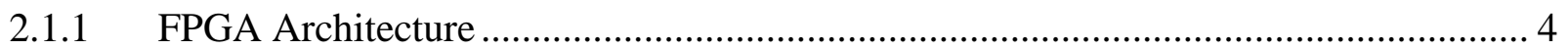

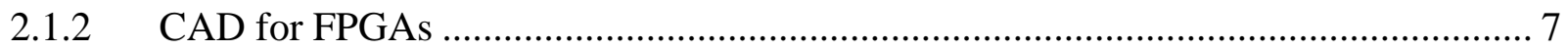

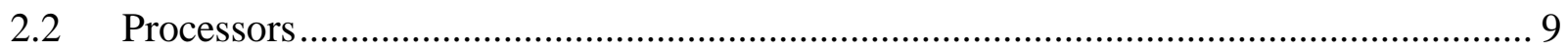

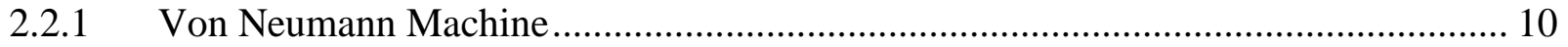

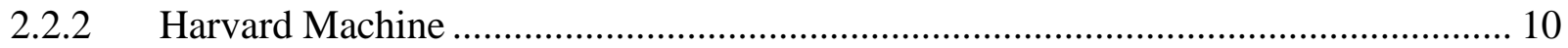

2.2.3 Typical Implementation of Computation on Processors .......................................... 11

2.3 Effects of functional unit complexity on dynamic energy consumption of processors . 12

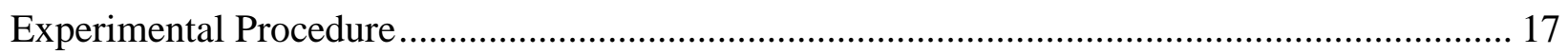

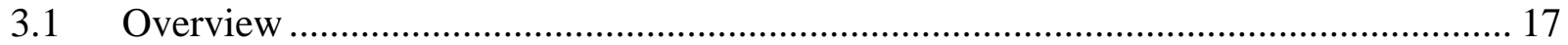

3.2 Sequential/Temporal Processing Energy ….......................................................... 18

3.2.1 Live Variable Analysis ........................................................................... 22

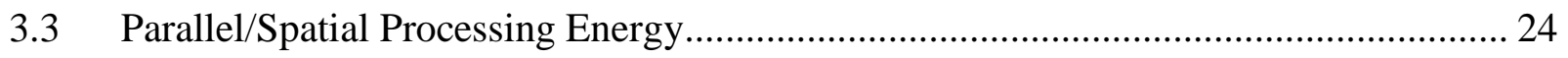

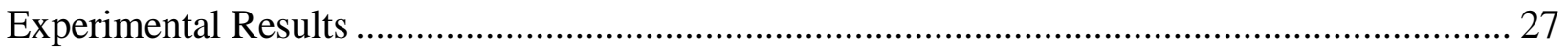

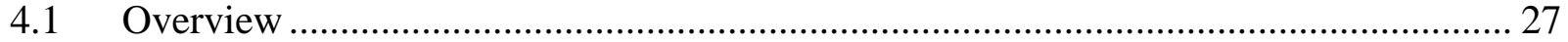

4.2 4-LUT Processor vs 4-LUT FPGA (Dynamic Energy) ............................................. 27

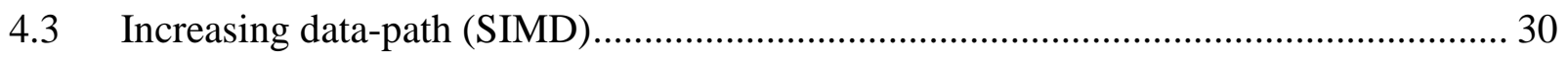

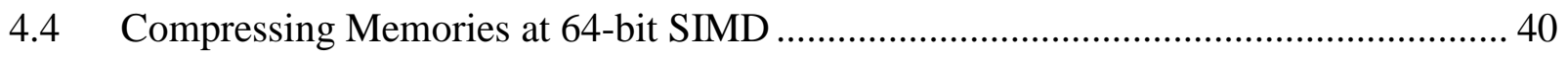

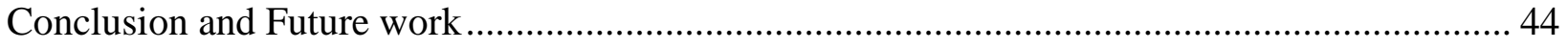

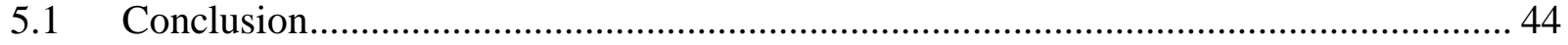




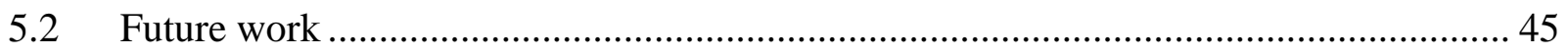

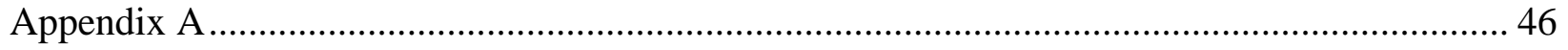

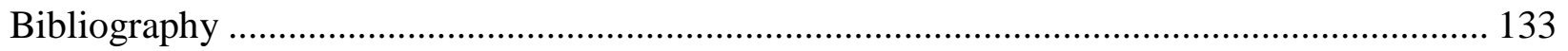

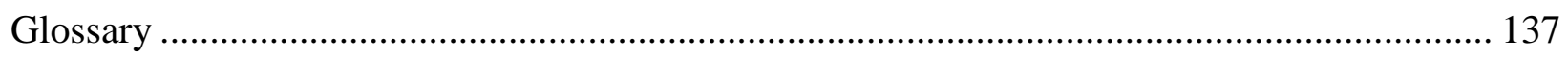




\section{List of Tables}

Table I. (7,2) Compressor Tree Dynamic Energy Consumption ........................................ 14

Table II. Dynamic Energy Consumption of MCNC Benchmarks 1bit (100\% Memory).......... 28

Table III. Energy Distribution for Processor ........................................................................ 29

Table IV. Dynamic Energy Consumption of MCNC Benchmarks 2bit (100\% Memory)........... 32

Table V. $\quad$ Dynamic Energy Consumption of MCNC Benchmarks 4bit (100\% Memory)........... 33

Table VI. Dynamic Energy Consumption of MCNC Benchmarks 8bit (100\% Memory).......... 34

Table VII. Dynamic Energy Consumption of MCNC Benchmarks 16bit (100\% Memory) ..... 35

Table VIII. Dynamic Energy Consumption of MCNC Benchmarks 32bit (100\% Memory) ..... 36

Table IX. Dynamic Energy Consumption of MCNC Benchmarks 64bit (100\% Memory)......... 37

Table X. Dynamic Energy Consumption of MCNC Benchmarks 128bit (100\% Memory) ....... 38

Table XI. Dynamic Energy Consumption of MCNC Benchmarks 256bit (100\% Memory) ....... 39

Table XII. Dynamic Energy at 64bit(s) 9\% Memory Size ................................................... 41 


\section{List of Figures}

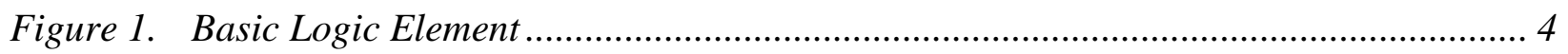

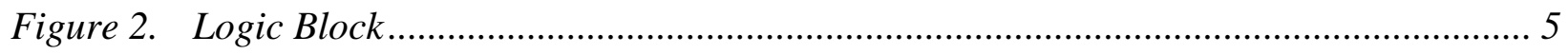

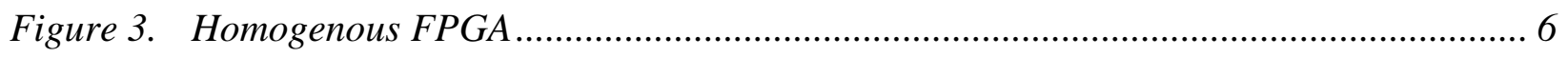

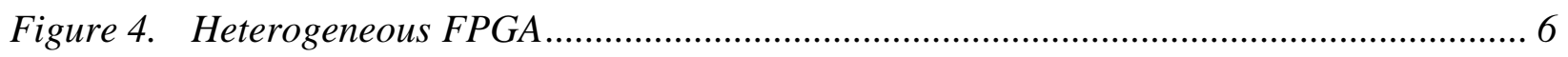

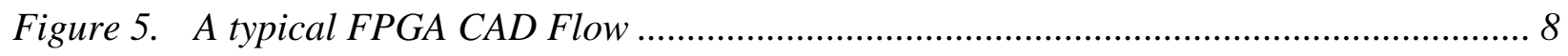

Figure 6. Von Neumann Machine …………………......................................................... 10

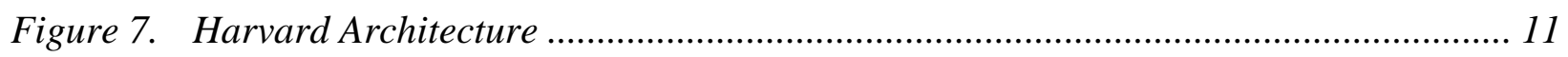

Figure 8. Typical Processor Computation Implementation …………………….................... 11

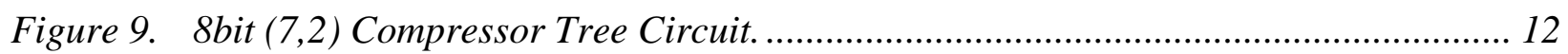

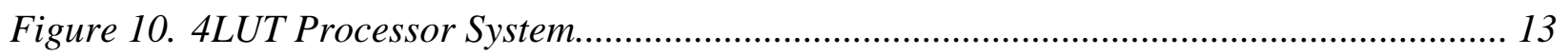

Figure 11. Experimental Procedure (CAD Flow) ............................................................ 17

Figure 12. Graph Representation of Circuit ...................................................................... 18

Figure 13. Linked List Representation of Circuit.................................................................. 19

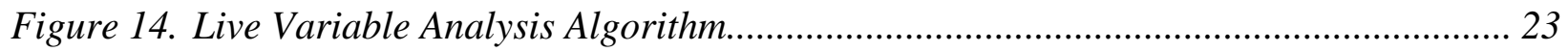

Figure 15. Definition of a minimum width transistor area ....................................................... 25

Figure 16. Ratio for MCNC Benchmarks (CPU/FPGA) …………………………................... 29

Figure 17. Dynamic Energy Ratio Vs Increasing Bit Width ...................................................... 30

Figure 18. Average Energy Ratio for MCNC Benchmarks at 64bit(s) ...................................... 40

Figure 19. Energy Distribution at 64bit (9\% Memory) ........................................................... 42

Figure 20. Dynamic Energy Consumption at 64bit(s) 9\% Memory ........................................... 43 


\section{Chapter 1}

\section{Introduction}

\subsection{Overview}

Microprocessors and Field-Programmable Gate Arrays (FPGAs) are the two main programmable platforms for implementing digital computation. The main computing elements in modern FPGAs are look-up tables (LUTs) and bit-configurable routing resources [1]. In combination, these resources can be effectively used to exploit bit-level data parallelism in order to deliver much higher performances and energy efficiency than today's state-of-the-art processors [2]. Modern microprocessors, however, are increasingly incorporating accelerators, such as GPUs [3], image processing and DSP cores [4], on the same die as CPUs. These accelerators function as advanced functional units and are much more complex in structure than the hard-blocks incorporated on an FPGA.

This difference in complexity is mainly due to the sequential execution nature of a processor and the parallel execution nature of an FPGA. In particular, on a processor, each functional unit is shared over many instructions so only a small number of functional units of each type are needed per processor. On an FPGA, on the other hand, many more hard-blocks of the same type are needed in order to exploit the parallelism. Consequently, FPGAs only can afford to use much simpler accelerators, such as multipliers, as hard-blocks while still maintaining their original parallel execution model [5]. 
Since the functional units and the hard-blocks are essentially ASICs, which are known to be much more energy efficient than reconfigurable LUTs [6], it is important to understand the impact of this difference in complexity on the energy efficiency of FPGAs and processors. To this end, this work first measures the dynamic energy required to execute MCNC benchmarks on a 4-LUTbased FPGA (spatial implementation). We then serialize the LUT configurations so each configuration is mapped into an instruction that can be executed by a 4-LUT-based sequential processor (temporal implementation). Using this serialized version of the LUT configurations, we measure the effect of increasing functional unit complexity on the dynamic energy efficiency of processors by relating the increase in functional unit complexity to the reduction in instruction and data memory size.

This work is related to [7]. It differs from previous work in two main aspects. First, this work uses a benchmark-based approach over the analytical model from previous work. In particular, the analytical model from [7] only considers homogenous activities, i.e. every single wire is switched at every clock cycle. This over-estimates dynamic energy consumption. In this work, we have provided more accurate results with a simulation based empirical approach of estimating heterogeneous activity (actual switching) on the nets by simulating MCNC benchmark circuits with random inputs.

Secondly, the work in [7] has only compared FPGAs to the simplest possible implementations of a processor (Single 4-LUT), which only use one LUT in its functional unit. It also shows that when using monolithic memories for both instruction and data, such a processor would consume much more dynamic energy than FPGAs [7].

Instructions based on LUT configurations, however, are extremely inefficient and often consume much more memory than it is required by conventional processor instructions - since real 
functional units are implemented in either custom ICs or ASICs and can include many hardwired instructions. The goal of this work is to help a designer making a selection between spatial (FPGA) or temporal (CPU) processing platform for implementing a computation based on dynamic energy efficiency. As a result, this work examines the trade-off between data and instruction memory size and the dynamic energy efficiency of processors. We specifically measure the amount of on-chip SRAM based instruction and data memory reduction that is needed from the LUT-based instructions in order for a processor to achieve the same dynamic energy consumption as a LUTbased homogenous FPGA. This result is particularly relevant in the age of dark silicon, where there is a trend for processors to include a variety of application-specific precompiled cores in order to increase their performance and efficiency [8]. Consequently, it is important to quantify the amount of memory reduction that a processor should achieve in order to be more energy efficient than regular LUT-based FPGAs.

\subsection{Thesis Organization}

The remainder of this thesis is structured as follows: Chapter 2 (Background and Motivation) provides architectural details about some of the platforms available for implementation computations such as FPGAs and CPUs. It also provides the motivation for measuring the effect of functional unit complexity on dynamic energy consumption of processors and FPGAs using an example of 8-bit wide (7:2) compressor tree circuit, Chapter 3 (Experimental Procedure) presents the procedure that is used to measure the dynamic energy consumption of the temporal representation of the MCNC benchmarks on a single 4-LUT based processor and the spatial 4LUT-based FPGA. Chapter 4 (Experimental Results) presents experimental results and Chapter 5 (Conclusion and Future work) concludes and future direction for further research. 


\section{Chapter 2}

\section{Background and Motivation}

\subsection{Field Programmable Gate Arrays}

FPGAs provide a hardware programmable platform to implement digital computations. Since their introduction in 1984, they have become a $\$ 30$ Billion (still growing) industry in 2016. They can provide a huge performance advantage over general purpose sequential processors by providing dedicated hardware such as application-customized data paths. They are re-programmable, and provide a competitive option against standard Cell ASIC development by reducing NRE costs and achieving faster time-to-market [9].

\subsubsection{FPGA Architecture}

In past few decades FPGA architecture has much evolved from containing a simple network of LUTs (Look-up Tables) and programmable routing circuits to complex network of LUTs and hardcore processing blocks, such as DSPs and BRAMs.

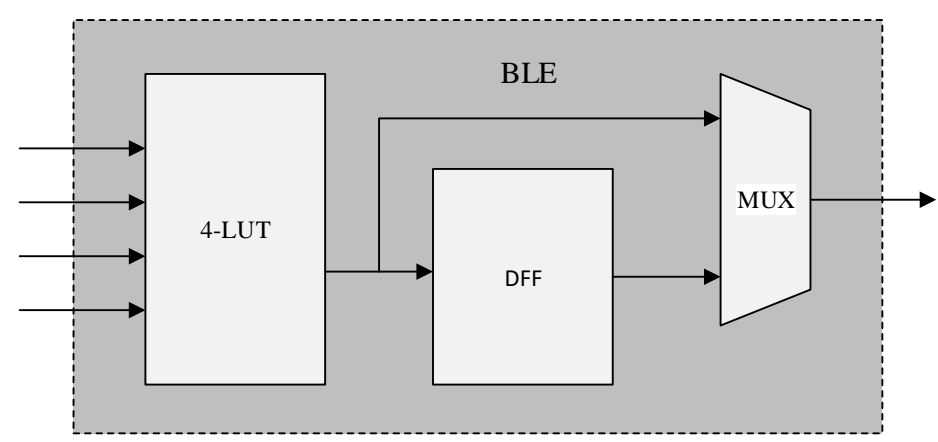

Figure 1. Basic Logic Element 
A typical FPGA contains k-input LUTs, DFFs (D-Flip Flops) and bit programmable routing circuits grouped into BLE (Basic Logic Element). Figure 1, shows a basic logic element of FPGA. DFF can used when output for a BLE needs to registered. This is known as 'soft logic' implementation on FPGA. In order to achieve higher densities on chip and reduce their overall delay, these BLEs are grouped together into 'clusters' also known as LB (Logic Blocks) [10]. A study has shown that using 4 BLEs inside a logic block can provide a 5-10\% reduction in area for FPGAs [11]. Also [12] shows that 4 input LUT is the most area efficient design mainly because, as the number of inputs for a single LUT grows the complexity of LUT grows exponentially making 4-LUT the most optimal design choice. This is one of the reason we choose 4-LUT for our research further explained in later chapters. Figure 2, shows a group of BLEs packed in a group to form a LB [11].

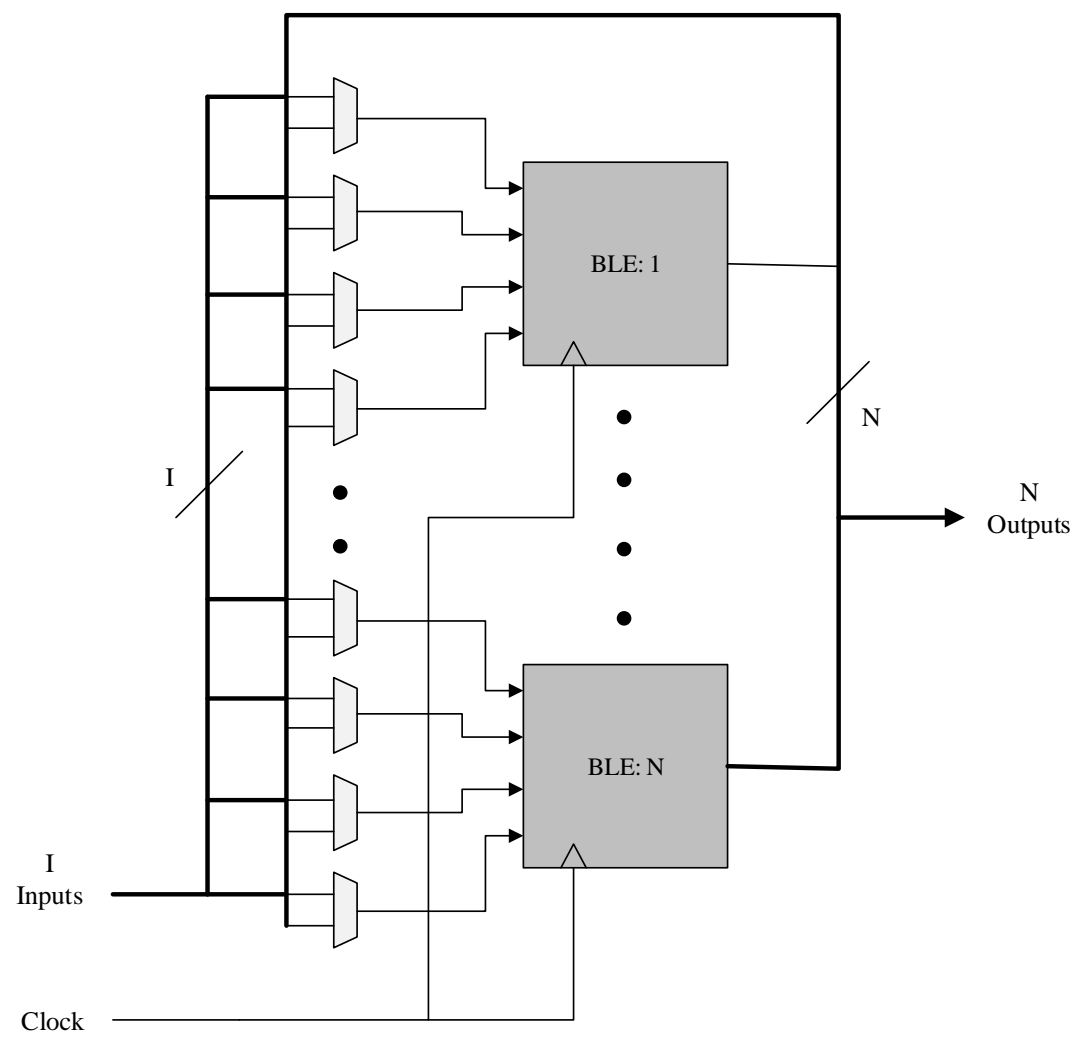

Figure 2. Logic Block 


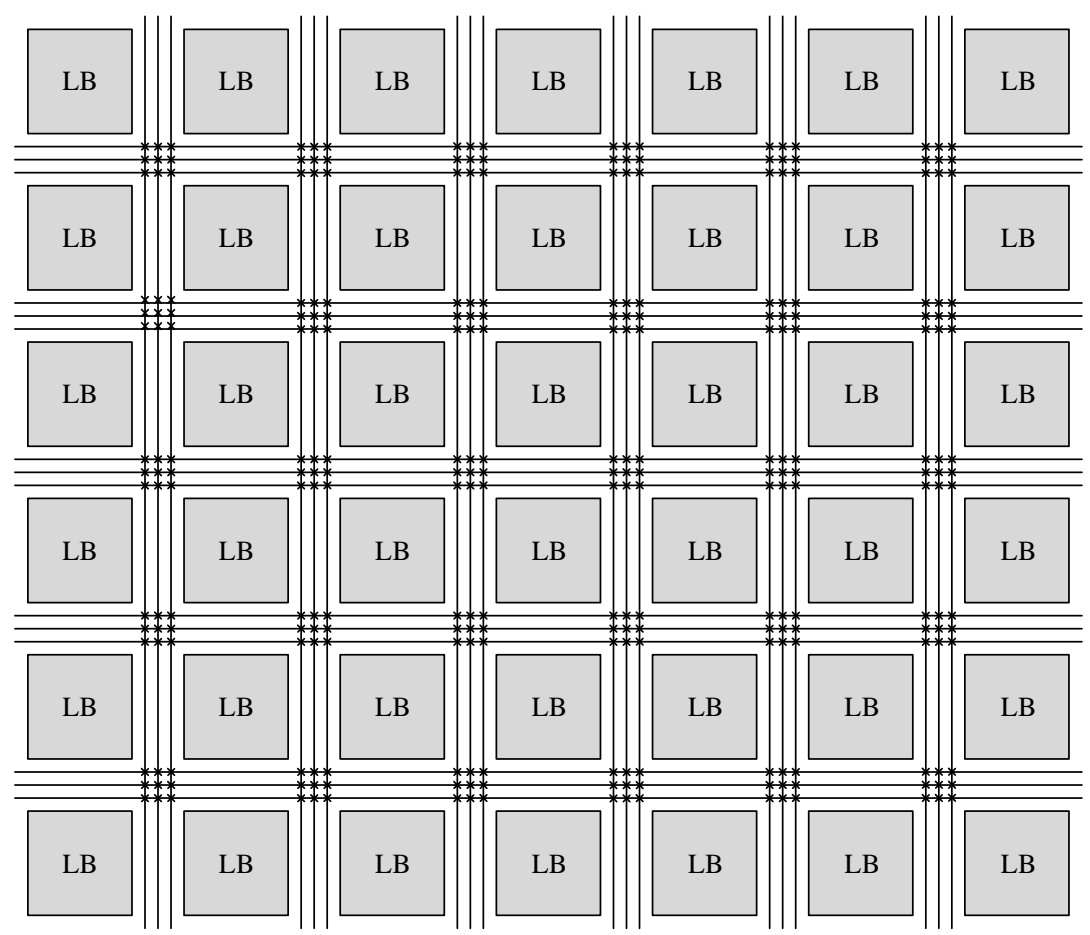

Figure 3. Homogenous FPGA

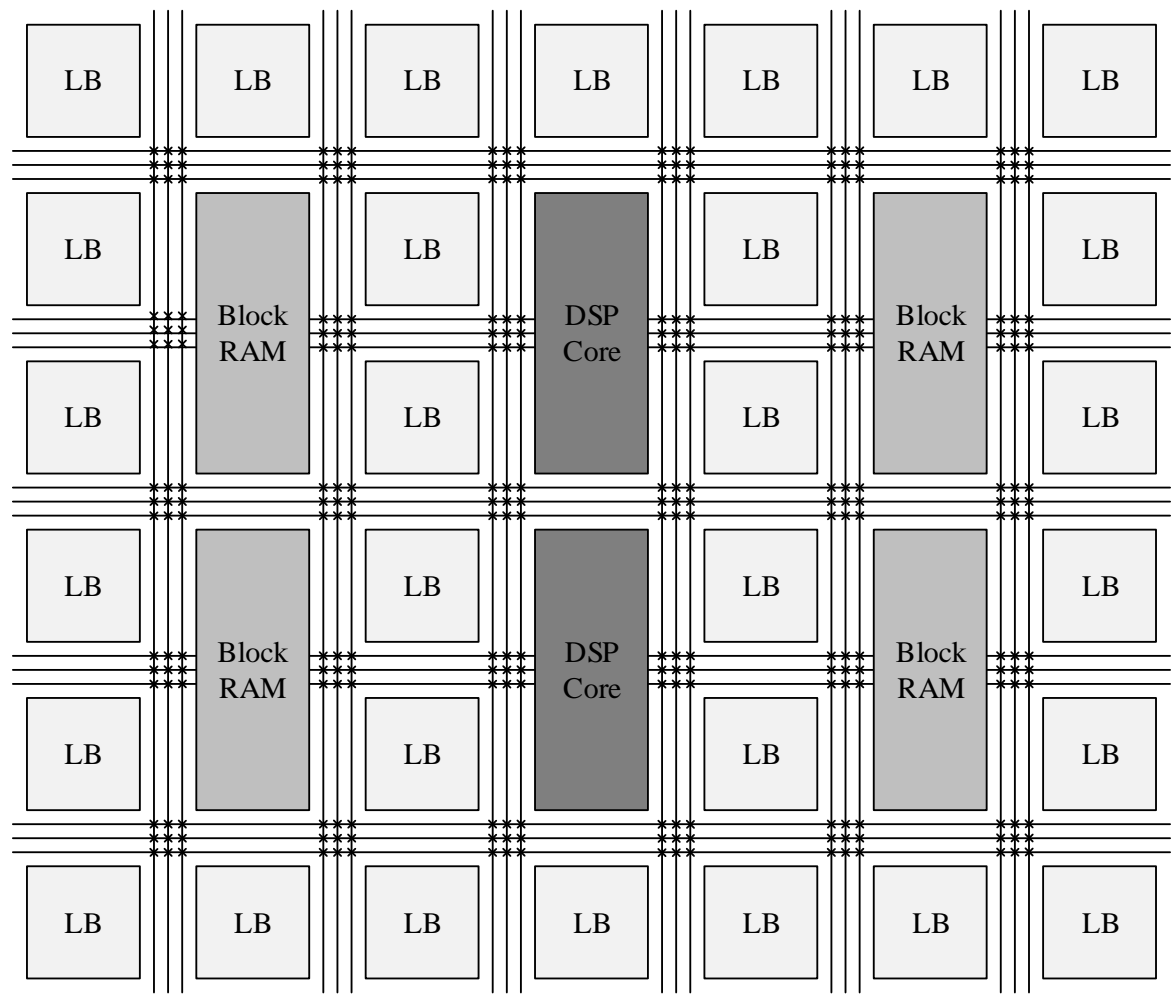

Figure 4. Heterogeneous FPGA 
Figure 3, shows a simple homogenous FPGA with LBs and routing network, however most modern FPGAs incorporate the 'hard' blocks such as DSPs or Block RAMs. Even though 'soft logic' can implement any type of digital circuit but having these 'hard' blocks on chip provide more efficiency by trading flexibility. A typical heterogeneous FPGA is shown in Figure 4.

\subsubsection{CAD for FPGAs}

In order to implement a digital circuit on FPGAs, a designer need to design and describe the circuit at a higher abstraction level which is either in HDL code or simply in graphical description. Before this design is ready to be implemented on FPGA, it needs to be converted into a low-level bit stream which can control the routing and logic switches inside the FPGA. This process requires a set of tools, and is known as 'CAD flow' for FPGAs. From design to device, these tools are required to make a lot of different decisions to implement the circuit more efficiently on the chip, while fulfilling all the design constrains such as area, delay and power [13]. Figure 5, shows a flow of these CAD tools [14].

\section{High Level Synthesis:}

A recent trend in FPGA CAD flow is the addition of HLS (High Level Synthesis), which provides the designer a highest level of abstraction by expressing the circuit algorithmically in more traditional programming languages such as C [15] and OpenCL [16]. Using this algorithmic description of HLS selects the appropriate hardware for implementation.

\section{Logic Synthesis:}

Logical synthesis consists of further 3 steps: elaboration, logic optimization and technology mapping. During elaboration, CAD converts the behavioral description of hardware into logical hardware description. Then logic optimization is then performed to improve the quality of resulting 
hardware by reducing area, delay or power. After the optimization performed here, mapping generates the netlist of circuit using primitive set of hardware available in FPGA such as LUTs, Flip-Flops and multipliers. A careful CAD implementation at earlier stages can reduce the over power consumption of FPGA, i.e. 7.6\% just because of power-aware technology mapping [17].
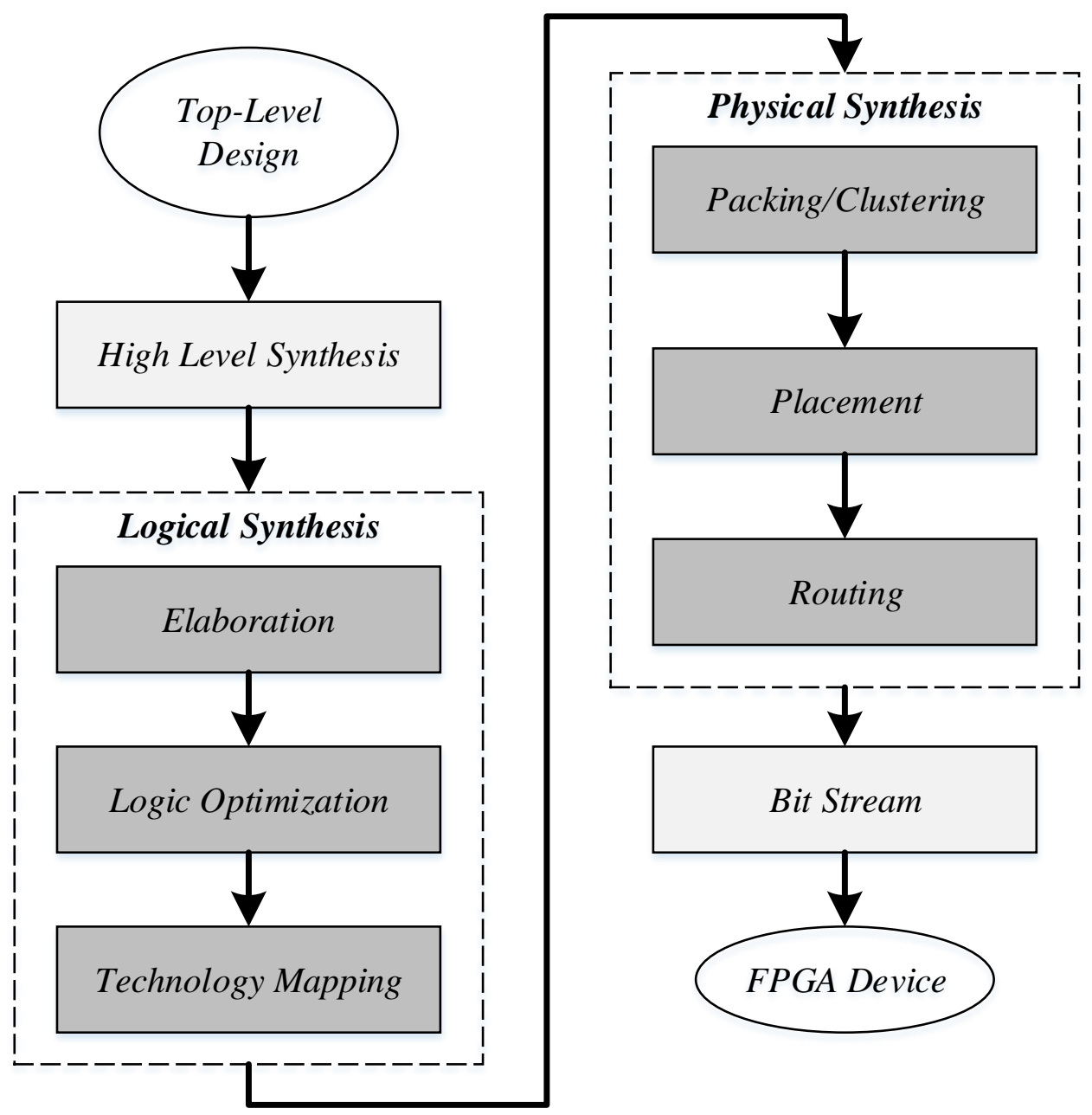

Figure 5. A typical FPGA CAD Flow

\section{Physical Synthesis:}

Physical synthesis is performed using various algorithms such as simulated annealing [18] [19] for placement and a variant of Lee's Algorithm [20] for routing [21]. This process consumes a great 
amount of compile time for FPGAs CAD flow, and the efficiency (power, speed and area) of final implementation very much depends on the efficiency of CAD tool being used. First step in physical synthesis is Clustering or Packing, which means grouping together the primitive hardware into the actual blocks of FPGA architecture. A good clustering algorithm also ensures that design is more efficiently routable and thus reducing compile time for later stages such as routing [22] [23].

Next step would Placement of these blocks on FPGA, it's one of the very important steps in FPGA implementation, as placement would also determine the accuracy of later steps such as routing. A better placement means less wires to be routed thus reducing the delay and power consumption of the final circuit. A bad placement might actually make a certain design un-routable on FPGA due to a limited number of routing resources available on the chip.

Last step in physical synthesis is Routing, which is similar to ASIC design flow, given the placement of different blocks at this point, it is determined how to connect these blocks using the routing resources available on FPGA.

Once the logical and physical synthesis is completed the final design is analyzed to ensure that it meets the design constraints such as delay and area. And finally the bit stream is generated which can be downloaded on the FPGA.

\subsection{Processors}

Sequential processors have a long and rich history as computational platforms than FPGAs. They are considered one of the most famous platforms for implementing computation. They provide software re-programmability, thus providing much flexibility than other solutions such as ASICs although which comes at a cost of performance. They provide a set of instructions, which are executed sequentially, using these set of instructions a programmer can write a program to 
implement any computation. The idea of stored program computers was first implemented in 1940s and used for military applications. There are two types of famous architectures for such computers, which differ in the way they store and access instructions and data; which is either in same or different physical memories.

\subsubsection{Von Neumann Machine}

Von Neumann architecture [24] stores the data and instructions for Central Processing Unit (CPU) in same physical memory and share the same bus to access them. According to Von Neumann the instructions are stored in a memory and extracted by the processor and then executed in a sequential manner, data stored in the memory is changed while the program is executed. Figure 6, shows a Von Neumann architecture [25].

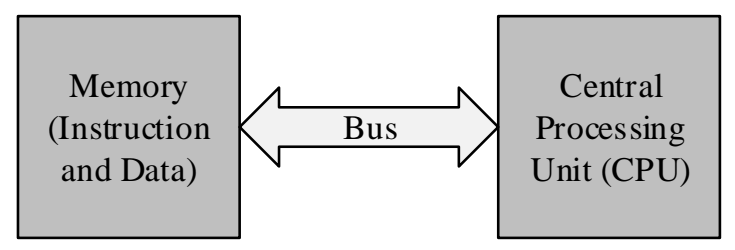

Figure 6. Von Neumann Machine

\subsubsection{Harvard Machine}

Harvard architecture differs from Von Neumann architectures by storing the instructions and data in separate memories connected with separate busses [26]. Instructions are executed sequentially by the processor, therefore instruction memory can be constructed as sequential access memory which requires less addressing circuitry than random access memories, thus reducing overall power consumption. We have chosen this architecture for our work to implement 4-LUT based processor, which is shown in Figure 10. A general form of Harvard machine is shown in Figure 7. 


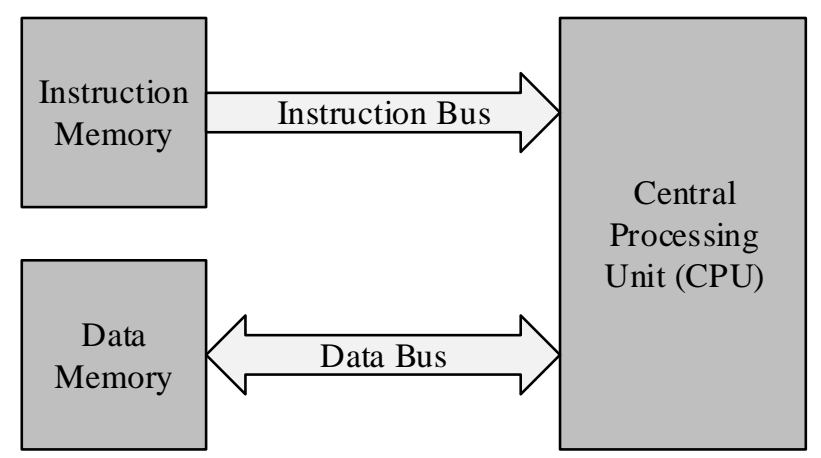

Figure 7. Harvard Architecture

\subsubsection{Typical Implementation of Computation on Processors}

Implementing computation using processors is much easier and requires less compile time than FPGAs. A typical flow to implement a computation, from a program written in higher level languages such as $\mathrm{C}$ and $\mathrm{C}++$ down to hardware is shown in Figure 8 [27].

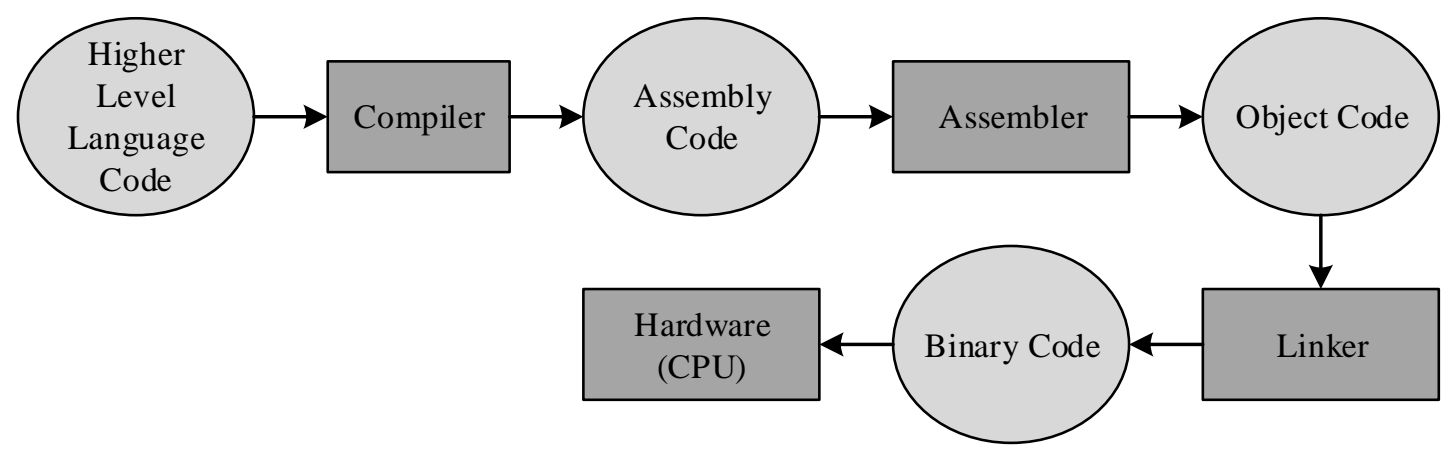

Figure 8. $\quad$ Typical Processor Computation Implementation

Where a compiler is a computer program which converts a higher level program, into low level assembly code, which is them read by assembler which converts it into object code which is the form of input a linker would take to generates bits of $0 s$ and $1 s$. As hardware can only understand the instructions in binary code, which is very hard for human programmers to program in, therefore these abstractions provide more ease to program for humans. 


\subsection{Effects of functional unit complexity on dynamic energy consumption of processors}

The dynamic energy consumption of a processor is strongly dependent on the logic complexity of its functional unit. In particular, consider measuring the dynamic energy required to implement an 8-bit wide (7:2) compressor tree [28], as shown in Figure 9, on a sequential processor. As shown, the circuit consists of a series of full adders (FA) acting as $(3,2)$ counters, and one can take the approach proposed in [7] to measure the dynamic energy consumed by the processor by first mapping the circuit into a set of 4-LUTs and then using a LUT-based processor as shown in Figure 10, to execute one LUT configuration at a time.

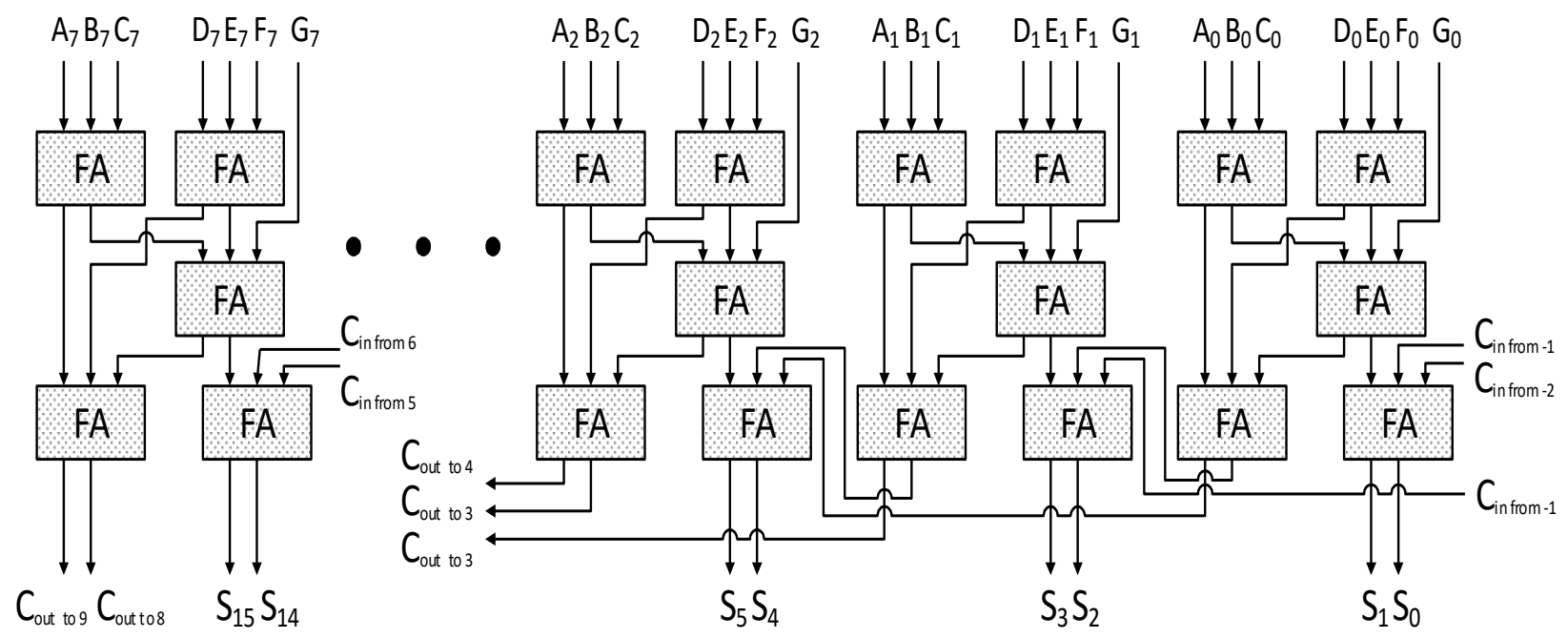

Figure 9. $\quad 8$ bit $(7,2)$ Compressor Tree Circuit.

The processor shown in Figure 10, consists of a functional unit containing a single 4-LUT and connected to two distinct monolithic memory blocks - a sequential access memory for storing instructions and a random access memory for storing data, implemented using 32nm CMOS process [7]. Note that in Table I, " $M$ " is the number of words in memory and " $W$ " is the width of 
each word in bits. As shown in Table I, $80 \times 46(M \times W)$ bits of instruction memory are required to encode the 4-LUT configurations, where there are 80 instructions (one for each 4-LUT) and each instruction consists of a 16-bit wide 4-LUT configuration bits and 56-bit wide addresses to indicate the location of the four (4-LUT inputs) and one (4-LUT output) in data memory. Also, $62 \times 1(M \times W)$ bits of data memory are needed to store all primary inputs and outputs of the circuit and to provide intermediate storage for internal LUT input and output values.

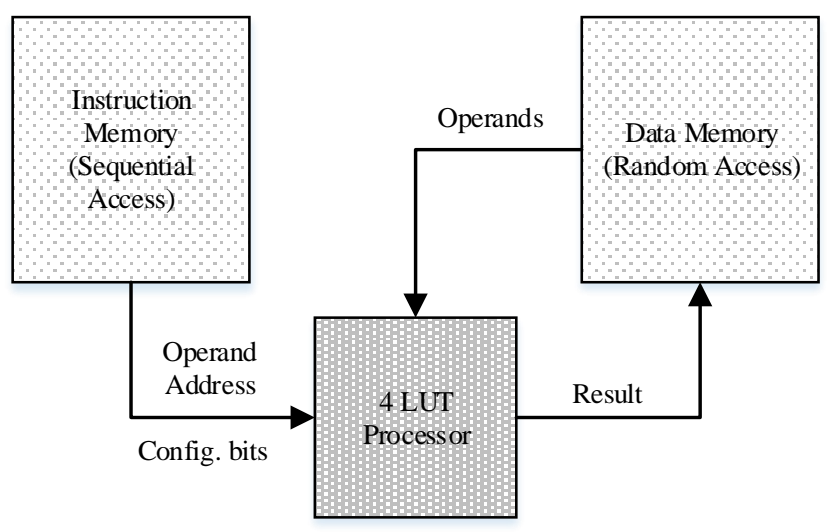

Figure 10. 4LUT Processor System

Once the size of the instruction and data memory is determined for the processor, one can measure the dynamic energy consumed by the processor by summing the total dynamic energy required to read the instructions from the instruction memory, total dynamic energy required to read (operands) and write (results) to the data memory and total dynamic energy required to evaluate each 4-LUT configuration [7].

In particular, the dynamic energy consumed for each read/write access to a memory block is a function of the size of the memory. In particular, for a fixed supply voltage, this energy is proportional to total wiring capacitance that is switched during the memory access and can be 
estimated using the following two equations for sequential and random access memory [7], respectively,

$$
\begin{aligned}
& C_{\text {Random }}(W, M)=\left(\log _{2}(M)+2(2 W+2)\right) \sqrt{W M A_{b i t}} C_{u} \\
& C_{\text {sequential }}(W, M)=2(2 W+1) \sqrt{W M A_{\text {bit }}} C_{u}
\end{aligned}
$$

Note that in Equation (1) and Equation (2), " $M$ " is total number of words in the memory block, " $W$ " is the width of each word, " $A_{b i t}$ " is the layout area for a one SRAM cell (estimated in [7] as $140 f^{2}$ for a dense SRAM cell, where $f$ is the feature size), and $C_{u}$ is $6.4 \times 10^{-18}$ Farads $(\mathrm{F})$, the wiring capacitance per feature size for minimum width wires.

Table I. $\quad(7,2)$ Compressor Tree Dynamic Energy Consumption

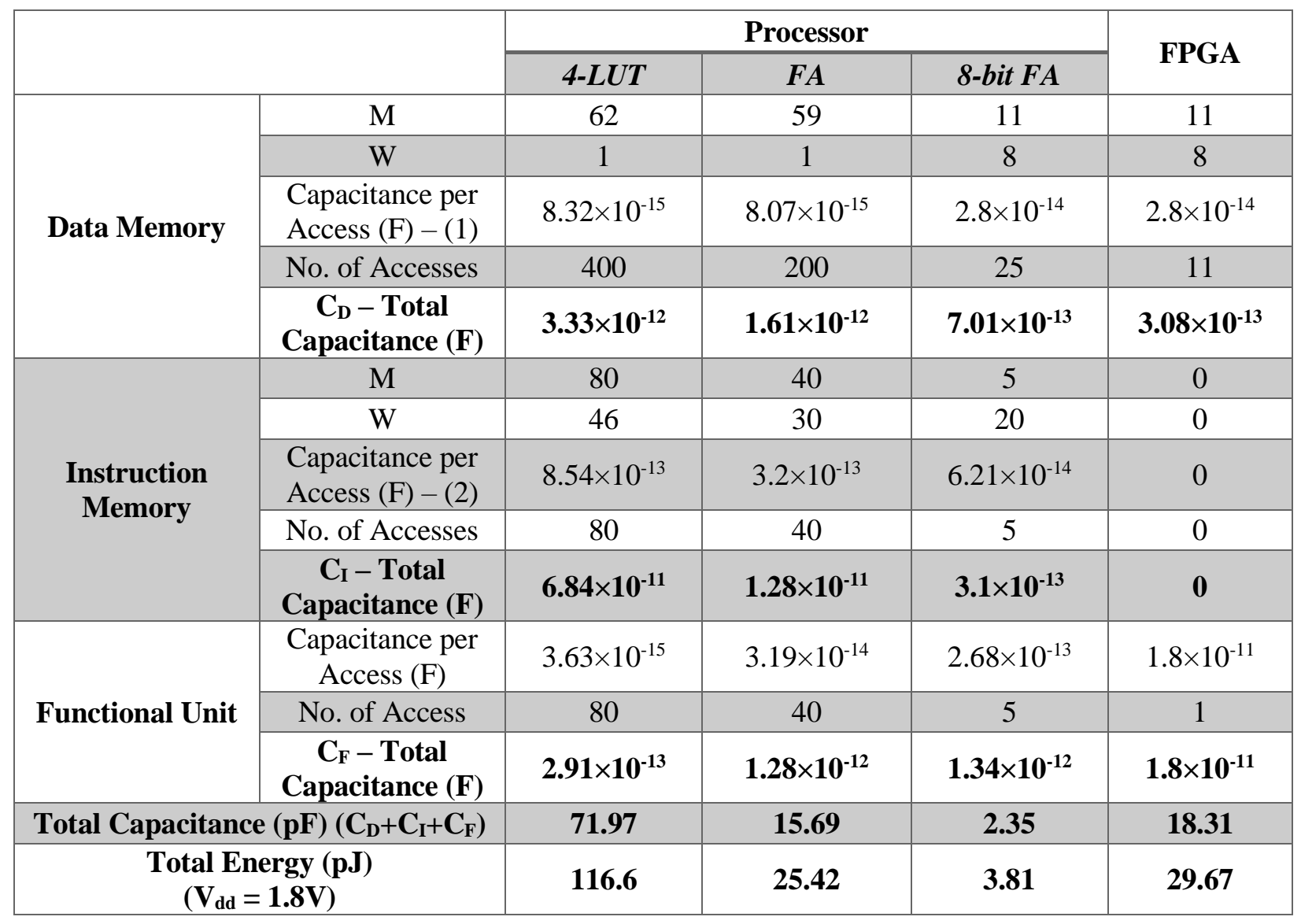


Table I presents the results obtained from the calculation. We use Equation (1) and Equation (2) to calculate the dynamic energy required to access instruction (sequential access) and data (random access) memory. In addition, we also estimate the 4-LUT evaluation energy using Equation (1) by treating the 4-LUT functional unit as a $16 \times 1(M \times W)$ bit random access memory. As shown in Table I, overall the 4-LUT processor is required to switch $71.97(\mathrm{pF})$ of capacitance in order to perform a full set of calculations of the (7:2) compressor tree. Assuming 1.8(V) supply voltage $\left(V_{d d}\right)$, this capacitance corresponds to $116.6(\mathrm{pJ})$ of total dynamic energy, calculated using Equation (3) [29].

$$
E_{\text {Dynamic }}=0.5 C V_{d d}^{2}
$$

It is important to note that this LUT-based functional unit is extremely inefficient since LUTs provide too fine-grain level of configuration. For example, we can replace the 4-LUT based functional unit by a full adder (FA) implemented on ASIC. As shown by column 3 (column Processor: FA) of Table I, the new processor has significantly reduced instruction memory size as well as significantly reduced the number of accesses to both the instruction and data memory. This leads to a significant reduction in total capacitance switched to access data and instruction memory from $3.33(\mathrm{pF})$ and $68.4(\mathrm{pF})$ to $1.61(\mathrm{pF})$ and $12.8(\mathrm{pF})$, respectively, and results in the reduction of total dynamic energy from $116.6(\mathrm{pJ})$ to $25.42(\mathrm{pJ})$.

Note that, we assume the FA (and the 8-bit FA) functional unit is implemented in ASIC and its energy is estimated using VPR 4.30 place and route result [30] and converted to ASIC energy using the methodology from [6] by assuming ASIC implementations consume 1/14th of the energy of FPGA implementations. It would be an over estimation as a careful ASIC implementation optimized for dynamic energy for such small circuits can be more efficient but for consistency and 
to keep the model simple for calculations we use the approach described in [6] throughout this work.

Extending the data-path width per instruction can further reduce the size of the instruction memory and the number of accesses to both the instruction and data memory. As shown by column 5 (column - Processor: 8-bit FA) of Table I, the total dynamic energy consumption is reduced to 3.81(pJ) for a processor with 8-bit wide full adder as its functional unit.

Table I, also lists the total dynamic energy consumed by (7:2) compressor tree placed and routed on a 4-LUT FPGA using VPR 4.30. The total logic and routing capacitance switched for executing one set of compressor tree calculations are then calculated and shown in column 6 (column FPGA) of Table I. The calculation assumes the primary inputs and outputs of the FPGA are stored in random access memory. The energy required to read configuration bit stream for FPGA was deliberately ignored due to the fact that this energy has very little contribution in total energy and can be amortized on large number of computations FPGAs perform after being configured once. Compared to the processor with 8-bit wide FA (implemented in ASIC) as its functional unit, the FPGA implementation consumes $7.7 \times$ more dynamic energy, while the FPGA consumes $3.92 \times$ less dynamic energy than the single 4-LUT processor implementation.

Given that processors can gain energy efficiency by using more specialized functional units in order to reduce the number of accesses and size of both instruction and data memory, the energy required to perform the same set of functions on a processor is strongly dependent on the complexity of the functional units that the processor contains. Consequently, for the remainder of this thesis, we measure the amount of instruction and data memory reduction that is required in order for a processor to become more dynamic energy efficient than LUT-based FPGAs. 


\section{Chapter 3}

\section{Experimental Procedure}

\subsection{Overview}

As in [7], wire-capacitance-based models are used in this work to measure the dynamic energy consumption of processors and FPGAs. In particular, for a processor, we measure the dynamic energy consumed by the processor for accessing its instruction memory, its data memory and its functional units. For an FPGA, we measure both the dynamic energy consumed by the LUT evaluations and the routing network. Finally, ASIC energy is estimated as 1/14th of FPGA energy [6]. The detailed experimental procedure that was used to measure the dynamic energy consumption of the MCNC benchmarks on a processor with a 4-LUT-based functional unit is presented in Section 3.2, and the experimental procedure used to measure the dynamic energy consumption of the same benchmarks on an FPGA is presented in Section 3.3.

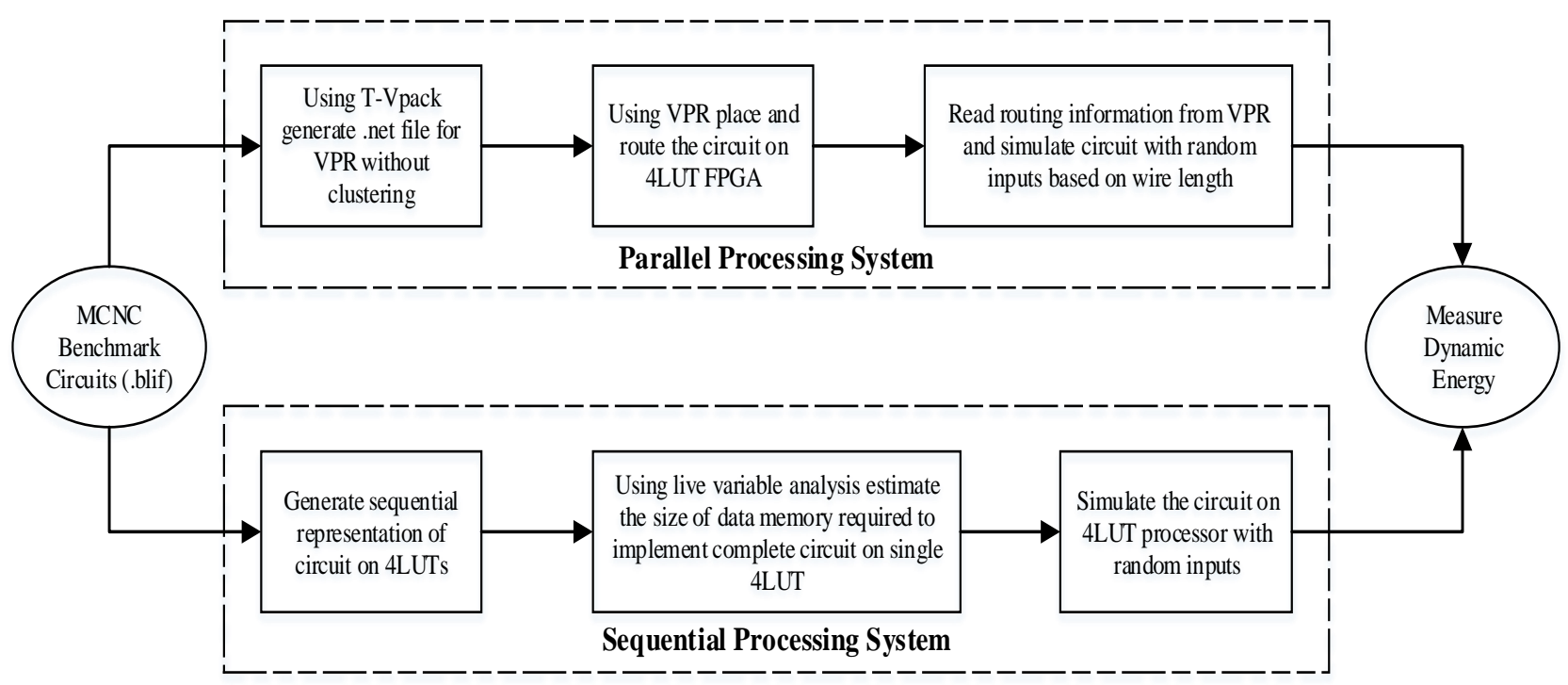

Figure 11. Experimental Procedure (CAD Flow) 


\subsection{Sequential/Temporal Processing Energy}

The CAD flow for estimating the dynamic energy required to execute the MCNC benchmarks on a sequential processing system is shown in Figure 11. In particular, we first map each 4-LUTbased FPGA circuit onto the 4-LUT-based processor as shown in Figure 10. T-Vpack [30] is first used to read each MCNC benchmark circuit in BLIF (Berkeley Logic Interchange Format) [31]. Once read, our tool first creates a directed graph representation of the circuit and then map the directed graph into a sequential temporal representation through breath-first search [32]. In particular, we schedule each LUT into a sequential execution sequence only if the sources of all inputs to the LUT have been scheduled previously, in software terms satisfying the variable dependencies in the program.

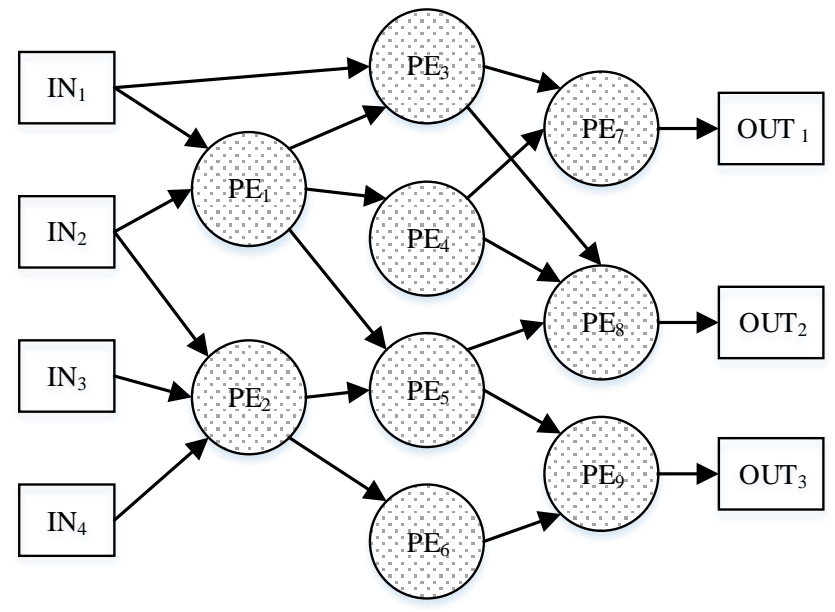

Figure 12. Graph Representation of Circuit

Figure 12, and Figure 13, show an example of such a schedule. In particular, Figure 12, shows the directed graph representation of a circuit. Here the rectangular nodes represent the primary inputs, outputs from registers, primary outputs and inputs to registers. Circular nodes represent single 4LUT-based processing elements "PEx", each containing only one 4-LUT configuration. The 
scheduler does a breath-first search through the graph and schedules primary inputs and register outputs first. Then each LUT is scheduled only if all nodes that generate the input edges to the LUT have already been scheduled before and the final sequential schedule is shown in Figure 13. Note that in Figure 13, the data memory location associated with $\mathrm{OUT}_{1}, \mathrm{OUT}_{2}$ and $\mathrm{OUT}_{3}$ will be updated as part of $\mathrm{PE}_{7}, \mathrm{PE}_{8}$ and $\mathrm{PE}_{9}$ execution respectively. Also, any registered outputs for LUTs (DFFs) are also scheduled before all Processing Elements (PEs). For the very first simulation cycle, they are considered to have a random value, which will be updated to the actual LUT result after each later cycle during simulation. Also note that in Figure 13, $t_{1}$ to t9 represents different times during execution. For example, at $t_{1}$ the LUT is configured as $\mathrm{PE}_{1}$ and after evaluation it is reconfigured as $\mathrm{PE}_{2}$ for second evaluation in $\mathrm{t}_{2}$, it is then reconfigured as $\mathrm{PE}_{3}$ for evaluation in $\mathrm{t}_{3}$, and so on.

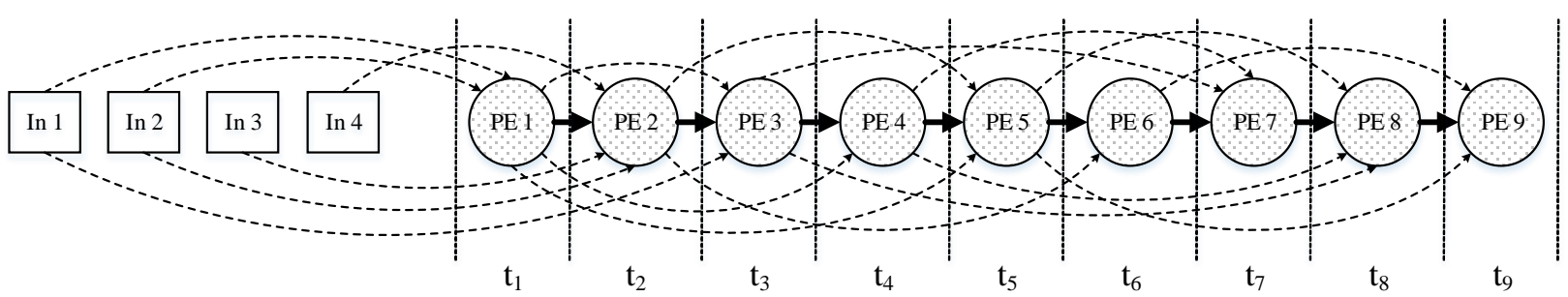

Figure 13. Linked List Representation of Circuit

In order to measure the dynamic energy consumed by the 4-LUT processor system, we perform a cycle by cycle simulation. During each cycle, the processor follows a traditional fetch, decode, execute and store cycle by first reading an instruction from the instruction memory. The instruction contains a 16-bit LUT configuration and five addresses indicating the location of the four inputs to the LUT and the one output of the LUT in data memory. Based on these addresses, the processor 
then reads the operands of the instruction from data memory. The 4-LUT instruction is then evaluated and the output written back to data memory.

Consequently, during each execution cycle, the processor would read the instruction memory once and read data memory for the maximum of four times and write to data memory once. Note that the number of read access to the data memory is the instruction dependent. Finally, we calculate the total dynamic energy consumed by the 4-LUT processor in each cycle by summing the dynamic energy consumed, to access the instruction memory, the data memory and the energy required to evaluate the 4-LUT functional unit as shown by Equation (4).

$$
E_{\text {Total }}=E_{\text {Data Memory }}+E_{\text {Instruction Memory }}+E_{\text {Functional Unit }}
$$

Dynamic power consumed by a circuit is directly proportional to the amount of capacitance being charged and discharged during its operation. We assume instruction memory is implemented in sequential access memory, as instructions are executed in strictly sequential manner and sequential access requires less energy than random access due to reduced decoding circuitry. We measure the capacitance consumed by the instruction memory based on Equation (2). Note that in order to estimate the capacitance, we need the size of the instruction memory in terms of the total number of memory words $\left(M_{\text {Instruction }}\right)$ and the width of each memory word in bits $\left(W_{\text {Instruction }}\right)$.

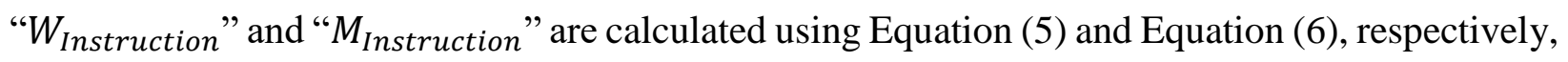

$$
\begin{aligned}
& W_{\text {Instruction }}=2^{k}+\operatorname{ceil}\left(5 \log _{2}\left(M_{\text {Data }}\right)\right) \\
& M_{\text {Instruction }}=N_{\text {LUTS }}
\end{aligned}
$$

where " $k$ " is the size of the LUT, " $M_{\text {Data }}$ " is the number of words contained in data memory, " $N_{\text {LUTS }}$ " is the total number of LUTs in each circuit and "ceil" means ceiling function. "W" and 
" $M$ " in Equation (2) is then set to " $W_{\text {Instruction" }}$ and " $M_{\text {Instruction" }}$ ", respectively, to calculate dynamic energy per access for the instruction memory.

Similarly, the capacitance consumed by data memory is measured using Equation (1). Note that LUT output is always a 1 bit wide therefore " $W_{\text {Data }}$ " is always equal to 1 . " $M_{\text {Data }}$ " is computed based on our live variable analysis results, explained in Section 3.2.1.

In particular, since data memory is implemented in random access memory, we can minimize the size of data memory by re-using the memory words containing data that are no longer required by the program (dead variables). Therefore, to measure the accurate size of the required data memory, we used live variable analysis algorithm [33] to eliminate dead variables after the execution of each instruction and measure " $M_{\text {Data }}$ " as the largest required memory size during the execution of an entire program. " $W$ " and " $M$ " in Equation (1) are then set to " $W_{D a t a " ~}$ "and " $M_{D a t a}$ " respectively to estimate to dynamic energy required for each access to the data memory.

For a single 4-LUT as a functional unit, we estimate the capacitance consumed by the functional unit itself also using Equation (1). Note that during each simulation cycle, each 4-LUT will be accessed twice, containing 1 write and 1 read access. First the 4-LUT is re-configured (1 write access) and then evaluated (1 read access) for each instruction. In particular, for the write access, we assume the write circuitry is implemented using random access memory containing 116 -bit word by setting " $W$ " to 16 and " $M$ " to 1 . For read access, we assume the read circuitry is implemented using the same SRAM cells but organized into 16 1-bit wide words by setting " $W$ " to 1 and " $M$ " to 16. 


\subsubsection{Live Variable Analysis}

In order to measure the correct amount of data memory words " $M_{\text {Data" }}$ " which is required to perform a single operation on 4-LUT processor, we implemented an algorithm to estimate the minimum amount of data memory required. A naive way of selecting data memory, to execute the complete schedule in Figure 13, is to choose a random memory containing words " $M_{D a t a}$ " equal to the total number of LUTs " $N_{\text {LUTS }}$ " in the circuit. An efficient implementation would be to measure the highest number of intermediate values stored in the data memory at any moment of time, during program execution. The purpose of this algorithm is to accurately measure the "Mata" based on the maximum "alive" variables or intermediate values stored in data memory at any moment of time during program execution.

This algorithm starts by initially going through the schedule in reverse order (starting from the LUTs scheduled at the end and directly connected to OutPads of FPGA), it will update a list of pointers in each node of the linked-list as shown in Figure 13, by providing it a list of pointers to all nodes it is being connected to in the future.

Once each node is aware of its future connections, we can start traversing the linked-list again from the very first node in the schedule and create a list of all connections (live variables) for future nodes. Also at each node we check if that particular node is present in the list, if it is present then it is considered as dead. Figure 14, provides the flow chart for this algorithm. Largest size of this list at any particular time during this traversal would be the minimum amount of data memory required to perform a single operation on 4-LUT processor. 


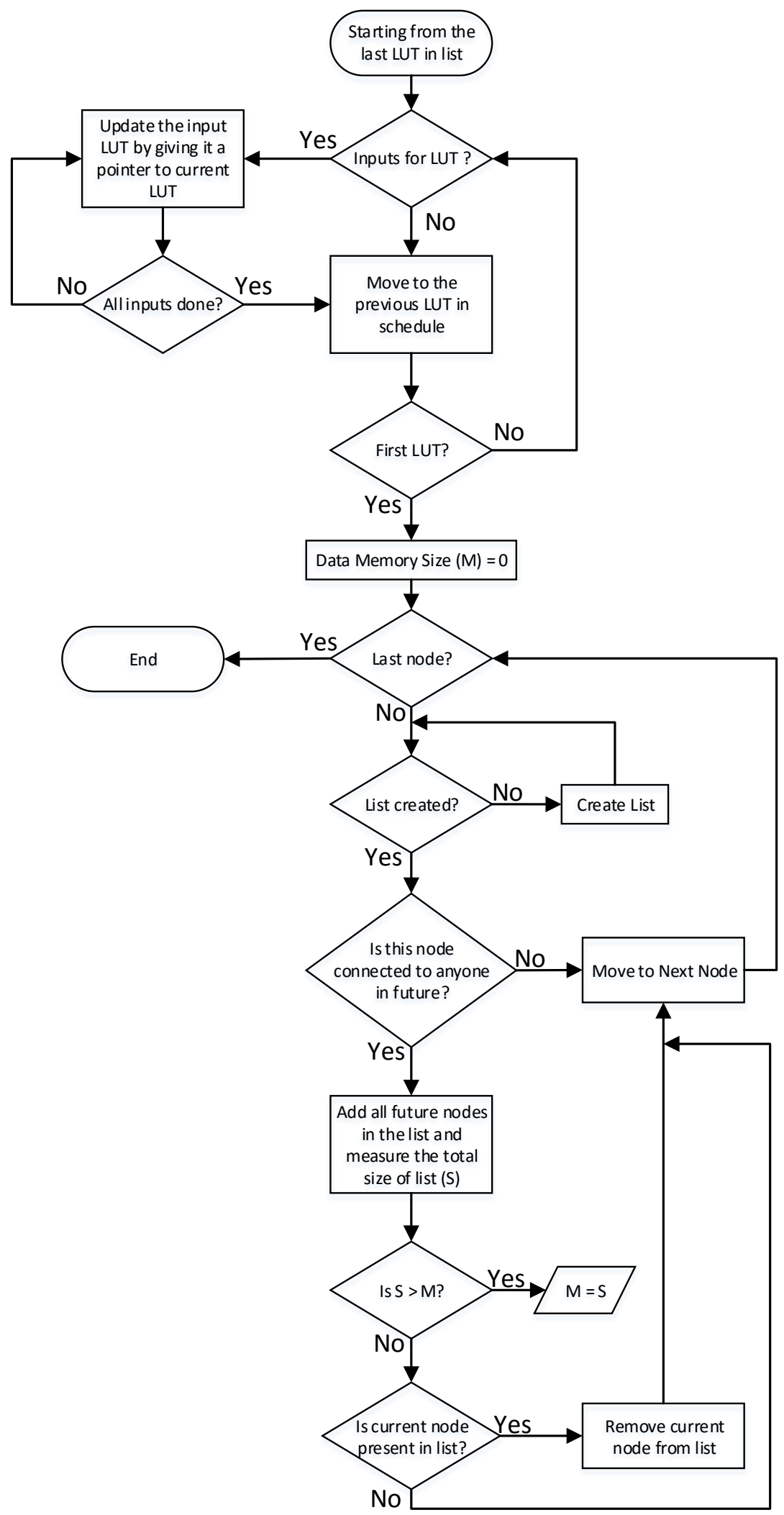

Figure 14. Live Variable Analysis Algorithm 


\subsection{Parallel/Spatial Processing Energy}

Our simulator uses the same schedule to measure the dynamic energy consumption of the 4-LUTbased FPGA that is used to measure the dynamic energy consumption of 4-LUT-based processor. Again a cycle by cycle simulation is performed to measure the actual net activities based on the results of individual LUT evaluations, using random primary inputs. Total dynamic energy consumed by the FPGA is then calculated as the sum of the energy required to switch the routing network and evaluate all LUTs as described in Equation (7).

$$
E_{\text {Total }(F P G A)}=E_{\text {LUTS Evaluations }}+E_{\text {Routing Network }}
$$

To measure LUT energy, we use Equation (1) by assuming each 4-LUT as a random access memory with 16 1-bit wide words. For routing energy, the CAD flow shown in Figure 11, is used. The VPR 4.30 tool chain [30] is used to map the MCNC benchmarks onto an island style FPGA where each logic block contains one 4-LUT and each routing track expands two logic blocks before being interrupted by routing switches. The VPR tools are then used to search for the minimum number of routing tracks that are needed to successfully route each circuit.

Assuming each FPGA tile is laid out in a square, the minimum width transistor area [34] per tile $\left(A_{m w t}\right)$ reported by the VPR tool is then used to calculate the length of routing tracks that is needed to span one FPGA tile. Minimum width transistor area means the layout area required to implement a smallest transistor in any process. VPR provides a generalized process free area estimation. Minimum width transistor area model for VPR assumes the layout area occupied by the transistor and distance required from its neighboring transistors, such as shown in Figure 15 from [34]. This area model is however improved by [35] by proving that VPR underestimates the actual layout area by a factor of $2.5 \times$, therefore Equation (8) adjusts the actual area by this factor. 


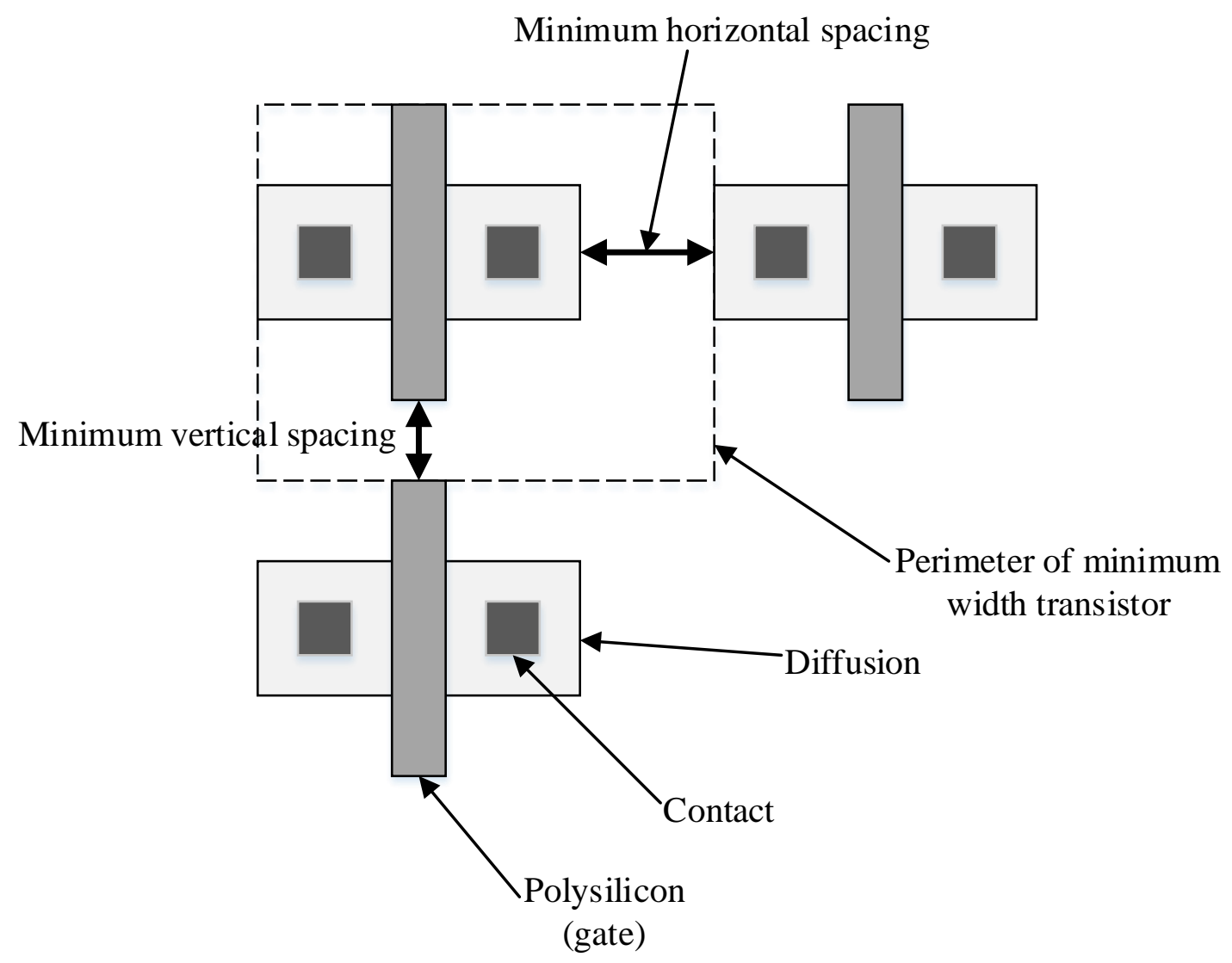

Figure 15. Definition of a minimum width transistor area.

The area is first converted into feature size based area using the lambda $(\lambda)$-based layout rules from [29] by assuming each minimum area transistor takes $40 f^{2}$ layout area. The feature size based area is then adjusted by a factor of $2.5 \times$ to account for the under-estimation of actual layout area by the minimum width transistor area model [35]. Finally, for each routing connection that corresponds to an edge in our routing schedule (shown as dashed lines in Figure 13,) the Manhattan distance $\left(D_{\text {Man }}\right)$ that the edge spans on the FPGA is then used to calculate the wiring capacitance

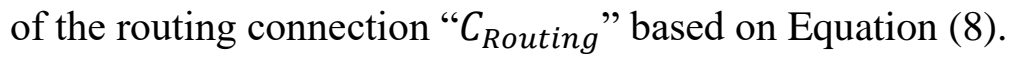

$$
C_{\text {Routing }}=D_{\text {Man }} \sqrt{2.5 A_{m w t} 40 f^{2}} C_{u}
$$


where $f$ is feature size and " $C_{u}$ " is $6.4 \times 10^{-18}(\mathrm{~F})$ at $32 \mathrm{~nm}$ process, the wiring capacitance per feature size for minimum width wires [7].

Note that energy required for configuring the LUTs and the routing resources are ignored by our simulation since once configured an FPGA is typically used over many cycles of computations and once amortized over all the cycles the configuration energy becomes negligible. 


\section{Chapter 4}

\section{Experimental Results}

\subsection{Overview}

We first present the dynamic energy consumption of the simple 4-LUT-based processor and compare the processor's energy consumption to the energy consumption of the 4-LUT-based FPGA for the MCNC benchmarks. The data-path width of the processor is then increased from 1bit to 256-bits, and its effects on the relative dynamic energy consumption between the processor and the FPGA is then measured. Finally, the effect of reducing the instruction and data memory sizes on the energy efficient of the processor over the FPGA is measured to account for the effect of replacing the 4-LUT based functional unit by more complex ASIC based functional units.

\subsection{4-LUT Processor vs 4-LUT FPGA (Dynamic Energy)}

Table II provides the results obtained from the experiment performed with a single 4-LUT as the functional unit for the processor. Column 1 lists the name of each benchmark. Column 2, 3, and 4 list the three components of the CPU dynamic energy consumption including the instruction memory, data memory, and functional unit dynamic energy consumption, respectively. Column 5 lists the total dynamic energy consumption of the CPU. The components of FPGA dynamic energy consumption are shown in column 6 and 7 including the routing energy consumption and 4-LUT evaluation energy consumption, respectively. The total FPGA dynamic energy consumption is shown in column 8. Finally, the ratio between the total CPU energy consumption and FPGA energy consumption is shown in column 9. As shown, on average for the MCNC benchmarks, the 4-LUT 
processor consumes $717 \times$ more dynamic energy to perform the same computation than the 4 -LUT FPGA.

Table II. Dynamic Energy Consumption of MCNC Benchmarks 1bit (100\% Memory)

\begin{tabular}{|c|c|c|c|c|c|c|c|c|}
\hline \multirow{2}{*}{$\begin{array}{c}\text { MCNC } \\
\text { Benchmark }\end{array}$} & \multicolumn{4}{|c|}{ Processor Dynamic Energy (nJ) } & \multicolumn{3}{|c|}{$\begin{array}{c}\text { FPGA Dynamic Energy } \\
(\mathbf{n J})\end{array}$} & \multirow{2}{*}{$\begin{array}{c}\text { Total } \\
\text { CPU/ } \\
\text { Total } \\
\text { FPGA }\end{array}$} \\
\hline & $\begin{array}{l}\text { Instruction } \\
\text { Memory }\end{array}$ & $\begin{array}{c}\text { Data } \\
\text { Memory }\end{array}$ & $\begin{array}{l}\text { Functional } \\
\text { Unit }\end{array}$ & $\begin{array}{l}\text { Total } \\
\text { CPU }\end{array}$ & Routing & $\begin{array}{c}4-L U T \\
\text { Evaluation }\end{array}$ & $\begin{array}{c}\text { Total } \\
\text { FPGA }\end{array}$ & \\
\hline alu4 & 224.69 & 4.15 & 0.11 & 228.95 & 1.37 & 0.11 & 1.48 & 154.58 \\
\hline apex2 & 307.97 & 5.70 & 0.14 & 313.81 & 1.85 & 0.14 & 1.99 & 157.36 \\
\hline apex4 & 169.65 & 2.95 & 0.05 & 172.65 & 0.62 & 0.10 & 0.72 & 240.26 \\
\hline bigkey & 301.90 & 6.19 & 0.08 & 308.17 & 0.98 & 0.13 & 1.11 & 277.65 \\
\hline clma & 3881.01 & 56.83 & 0.32 & 3938.16 & 3.90 & 0.63 & 4.53 & 869.43 \\
\hline des & 240.14 & 4.63 & 0.14 & 244.92 & 1.91 & 0.12 & 2.03 & 120.89 \\
\hline diffeq & 273.66 & 4.31 & 0.02 & 277.99 & 0.15 & 0.11 & 0.27 & 1038.92 \\
\hline dsip & 223.26 & 5.48 & 0.07 & 228.81 & 0.84 & 0.10 & 0.95 & 242.05 \\
\hline elliptic & 1306.20 & 17.19 & 0.05 & 1323.45 & 0.47 & 0.27 & 0.74 & 1796.47 \\
\hline $\operatorname{ex1010}$ & 131.33 & 2.14 & 0.05 & 133.52 & 0.59 & 0.08 & 0.67 & 199.32 \\
\hline ex5p & 1436.42 & 23.80 & 0.11 & 1460.33 & 1.13 & 0.35 & 1.47 & 990.53 \\
\hline frisk & 1212.78 & 15.12 & 0.04 & 1227.94 & 0.27 & 0.27 & 0.54 & 2275.50 \\
\hline misex3 & 197.59 & 3.70 & 0.09 & 201.37 & 1.09 & 0.11 & 1.19 & 169.01 \\
\hline pdc & 1425.66 & 25.58 & 0.15 & 1451.39 & 1.75 & 0.34 & 2.10 & 691.75 \\
\hline s298 & 322.18 & 5.58 & 0.04 & 327.79 & 0.40 & 0.15 & 0.54 & 605.07 \\
\hline s38417 & 2719.04 & 36.48 & 0.08 & 2755.61 & 0.67 & 0.46 & 1.13 & 2436.12 \\
\hline s38584.1 & 2753.42 & 39.72 & 0.09 & 2793.23 & 0.76 & 0.47 & 1.24 & 2259.65 \\
\hline seq & 277.03 & 4.85 & 0.12 & 282.00 & 1.54 & 0.13 & 1.67 & 169.03 \\
\hline spla & 938.94 & 16.56 & 0.12 & 955.62 & 1.40 & 0.28 & 1.68 & 569.66 \\
\hline tseng & 175.13 & 2.37 & 0.01 & 177.52 & 0.10 & 0.08 & 0.18 & 970.59 \\
\hline Average: & 925.90 & 14.17 & 0.09 & 940.16 & 1.09 & 0.22 & 1.31 & 717.13 \\
\hline
\end{tabular}


Table III presents the energy distribution among memories and the functional unit. As show in column 2 of Table III, majority of this energy (98.48\%) is consumed in reading the instructions from sequential memory.

Table III. Energy Distribution for Processor

\begin{tabular}{|l|c|c|c|}
\hline SIMD Bit(s)/Mem Size (\%) & $\mathbf{1 / 1 0 0}$ & $\mathbf{6 4 / 1 0 0}$ & $\mathbf{6 4 / 9}$ \\
\hline Instruction Memory (\%) & 98.48 & 36.44 & 34.27 \\
\hline Data Memory (\%) & 1.51 & 63.36 & 58.78 \\
\hline Functional Unit (\%) & 0.01 & 0.20 & 6.95 \\
\hline
\end{tabular}

\section{Processor vs FPGA 1-bit Width $100 \%$ Memory}

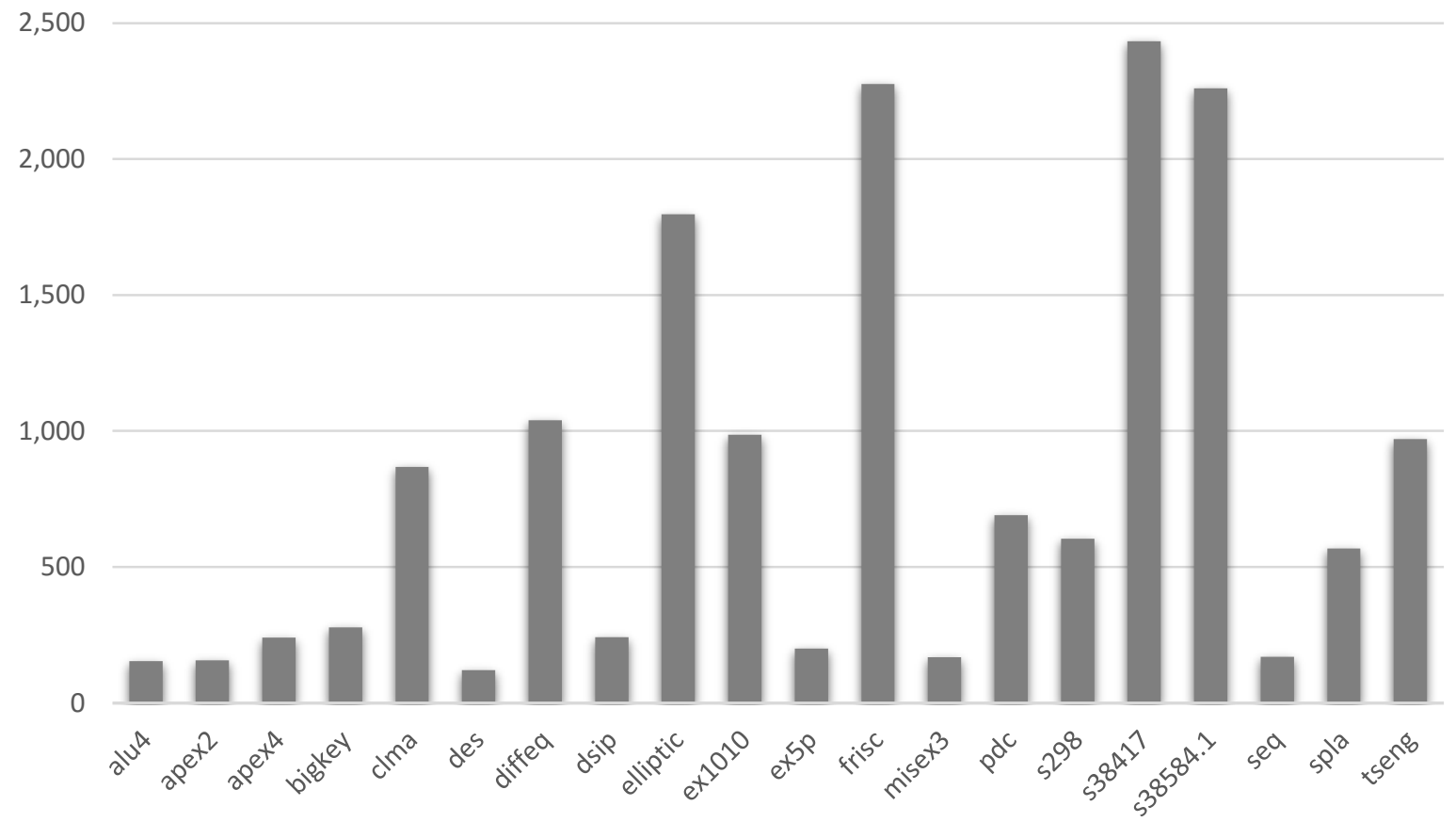

Figure 16. Ratio for MCNC Benchmarks (CPU/FPGA)

Figure 16, shows the difference between the energy consumption of CPU when using single 4LUT as processor and an island style FPGA for MCNC Benchmarks, placed and routed by VPR. 


\subsection{Increasing data-path (SIMD)}

Processors can increase their dynamic energy efficiency by sharing the same instruction on a set of data that is more than 1-bit wide. This property is known as SIMD, single instruction multiple data [7]. Storing a set of bits in the same word in data memory reduces the overall energy per bit required to address the word. FPGAs, however, require a complete replication of same the LUT network for each additional bit. Therefore, using a wider data-path should decrease the energy efficiency gap between the processor and the FPGA.

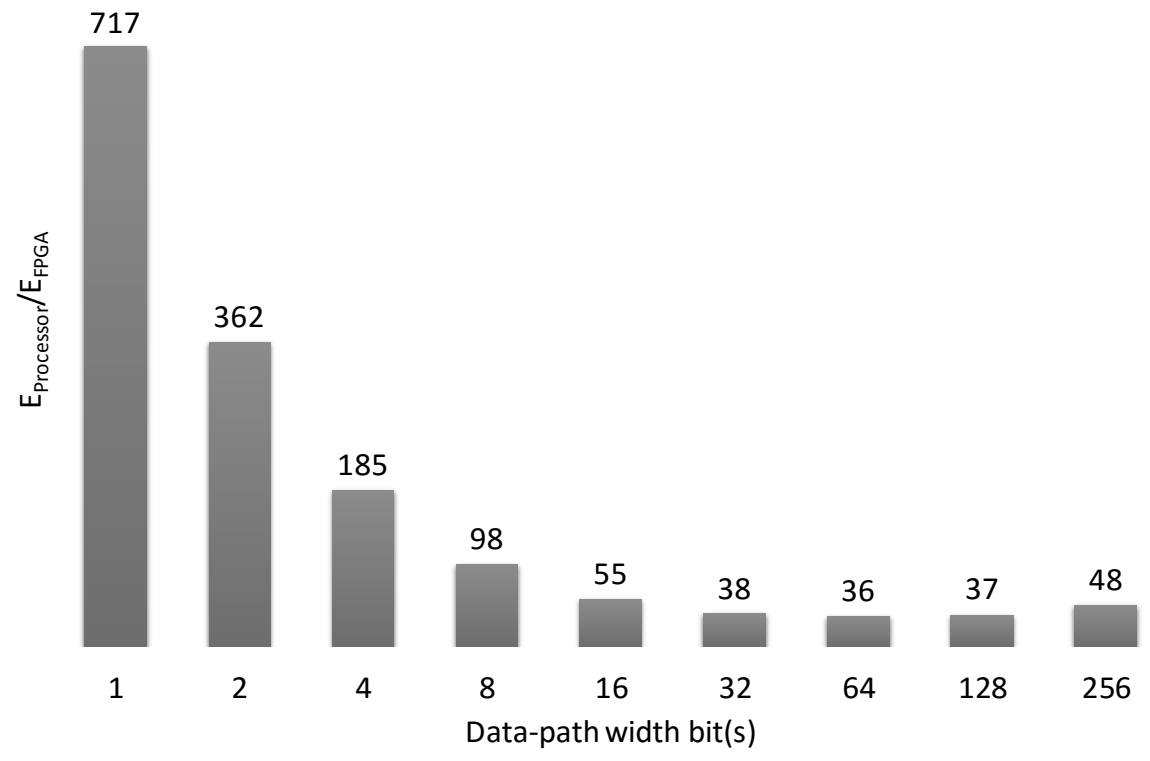

Figure 17. Dynamic Energy Ratio Vs Increasing Bit Width

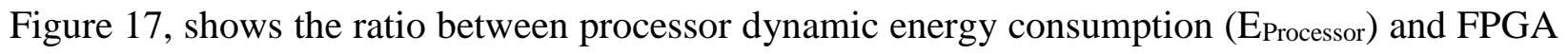
dynamic energy consumption ( $\left.\mathrm{E}_{\mathrm{FPGA}}\right)$ as a function of data path width. As shown, the ratio of the dynamic energy consumed by the processor and the FPGA decreases as the data-path width is increased. The ratio reaches a minimum at 64 bits, where the processor consumes $36 \times$ more energy than the FPGA. After 64-bit width, this ratio starts to increase due to the non-linear nature of the random access memory model as a function of bit width [7]. 
Note that at 1-bit wide data-path, the processor contains a single 4-LUT as its functional unit. However, for multiple bit wide data-paths, the processor contains one additional 4-LUT for each additional bit. This increases the per access dynamic energy consumed by the functional unit. Data memory energy also increases as its width increases. However, there is no change in dynamic energy consumption by the instruction memory. This behavior is summarized by column 3 of Table III, where, at 64-bit wide data-path width, the data memory consumes the most dynamic energy $(63.36 \%)$, instruction memory consumes $(36.44 \%)$ of the total dynamic energy and the functional unit still consumes a very small $(0.2 \%)$ but increasing amount of the total dynamic energy due to the reduction in the overall all dynamic energy consumption of the processor.

Table IV, Table V, Table VI, Table VII, Table VIII, Table IX, Table X and Table XI provides the energies in (n)Joules for all 20 MCNC Benchmarks, at 2, 4, 8, 16, 32, 64, 128 and 256 bits wide data paths, respectively. In each table; column 1, lists the MCNC Benchmark function; column 2, provides the energy consumed by instruction memory for processor; column 3, provides the energy consumed by data memory; column 4, provides the energy consumed by the array of 4-LUT(s) in functional units; column 5, lists the total energy consumed by CPU, while column 6 and 7 lists the FPGA energy and ratio of $E_{\text {Processor }}$ and $E_{F P G A}$. While last row provides the arithmetic mean for all 20 MCNC Benchmarks, except the last cell for 'Total CPU/ Total FPGA' provides the ratio between 'Arithmetic Mean of CPU' by 'Arithmetic Mean of FPGA'. 
Table IV. Dynamic Energy Consumption of MCNC Benchmarks 2bit (100\% Memory)

\begin{tabular}{|c|c|c|c|c|c|c|}
\hline \multirow{2}{*}{$\begin{array}{c}\text { MCNC } \\
\text { Benchmark }\end{array}$} & \multicolumn{4}{|c|}{ Processor Dynamic Energy (nJ) } & \multirow{2}{*}{$\begin{array}{c}\text { FPGA } \\
\text { Dynamic } \\
\text { Energy (nJ) }\end{array}$} & \multirow{2}{*}{$\begin{array}{c}\text { Total CPU/ } \\
\text { Total } \\
\text { FPGA }\end{array}$} \\
\hline & $\begin{array}{c}\text { Instruction } \\
\text { Memory }\end{array}$ & $\begin{array}{c}\text { Data } \\
\text { Memory }\end{array}$ & $\begin{array}{c}\text { Functional } \\
\text { Unit }\end{array}$ & $\begin{array}{l}\text { Total } \\
\text { CPU }\end{array}$ & & \\
\hline alu4 & 56.17 & 1.80 & 0.05 & 58.03 & 0.74 & 78.39 \\
\hline apex2 & 76.99 & 2.47 & 0.07 & 79.53 & 1.00 & 79.88 \\
\hline apex4 & 42.41 & 1.28 & 0.03 & 43.72 & 0.36 & 121.68 \\
\hline bigkey & 75.48 & 2.67 & 0.04 & 78.19 & 0.56 & 140.82 \\
\hline clma & 970.25 & 24.15 & 0.16 & 994.56 & 2.27 & 438.68 \\
\hline des & 60.04 & 2.01 & 0.07 & 62.12 & 1.01 & 61.35 \\
\hline diffeq & 68.42 & 1.87 & 0.01 & 70.29 & 0.13 & 525.57 \\
\hline dsip & 55.82 & 2.36 & 0.03 & 58.21 & 0.47 & 123.17 \\
\hline elliptic & 326.55 & 7.36 & 0.03 & 333.93 & 0.37 & 905.68 \\
\hline $\operatorname{ex1010}$ & 359.11 & 10.17 & 0.05 & 369.32 & 0.74 & 500.12 \\
\hline ex5p & 32.83 & 0.94 & 0.02 & 33.80 & 0.33 & 101.32 \\
\hline frisk & 303.20 & 6.49 & 0.02 & 309.70 & 0.27 & 1147.69 \\
\hline $\operatorname{misex} 3$ & 49.40 & 1.61 & 0.04 & 51.05 & 0.60 & 85.58 \\
\hline pdc & 356.41 & 10.93 & 0.07 & 367.42 & 1.05 & 350.70 \\
\hline s298 & 80.54 & 2.42 & 0.02 & 82.98 & 0.27 & 305.65 \\
\hline s38417 & 679.76 & 15.53 & 0.04 & 695.33 & 0.56 & 1236.22 \\
\hline s38584.1 & 688.35 & 16.87 & 0.04 & 705.27 & 0.62 & 1140.21 \\
\hline seq & 69.26 & 2.11 & 0.06 & 71.42 & 0.84 & 85.51 \\
\hline spla & 234.73 & 7.11 & 0.06 & 241.90 & 0.84 & 287.80 \\
\hline tseng & 43.78 & 1.03 & 0.01 & 44.82 & 0.09 & 490.35 \\
\hline Average: & 231.48 & 6.06 & 0.05 & 237.58 & 0.66 & 362.41 \\
\hline
\end{tabular}


Table V. Dynamic Energy Consumption of MCNC Benchmarks 4bit (100\% Memory)

\begin{tabular}{|c|c|c|c|c|c|c|}
\hline \multirow{2}{*}{$\begin{array}{c}\text { MCNC } \\
\text { Benchmark }\end{array}$} & \multicolumn{4}{|c|}{ Processor Dynamic Energy (nJ) } & \multirow{2}{*}{$\begin{array}{c}\text { FPGA } \\
\text { Dynamic } \\
\text { Energy }(\mathbf{n J})\end{array}$} & \multirow{2}{*}{$\begin{array}{c}\text { Total CPU/ } \\
\text { Total } \\
\text { FPGA }\end{array}$} \\
\hline & $\begin{array}{c}\text { Instruction } \\
\text { Memory }\end{array}$ & $\begin{array}{c}\text { Data } \\
\text { Memory }\end{array}$ & $\begin{array}{c}\text { Functional } \\
\text { Unit }\end{array}$ & $\begin{array}{l}\text { Total } \\
C P U\end{array}$ & & \\
\hline alu4 & 56.17 & 3.50 & 0.11 & 59.78 & 1.48 & 40.35 \\
\hline apex2 & 76.99 & 4.77 & 0.14 & 81.91 & 2.00 & 41.04 \\
\hline apex4 & 42.41 & 2.50 & 0.05 & 44.96 & 0.72 & 62.53 \\
\hline bigkey & 75.48 & 5.14 & 0.08 & 80.70 & 1.11 & 72.51 \\
\hline clma & 970.25 & 45.64 & 0.32 & 1016.21 & 4.53 & 224.28 \\
\hline des & 60.04 & 3.90 & 0.14 & 64.08 & 2.03 & 31.62 \\
\hline diffeq & 68.42 & 3.61 & 0.02 & 72.04 & 0.27 & 269.21 \\
\hline dsip & 55.82 & 4.55 & 0.07 & 60.43 & 0.94 & 64.01 \\
\hline elliptic & 326.55 & 14.02 & 0.05 & 340.62 & 0.74 & 462.12 \\
\hline $\operatorname{ex1010}$ & 359.11 & 19.33 & 0.11 & 378.54 & 1.48 & 256.22 \\
\hline $\operatorname{ex5p}$ & 32.83 & 1.84 & 0.05 & 34.72 & 0.67 & 51.96 \\
\hline frisk & 303.20 & 12.41 & 0.04 & 315.64 & 0.54 & 584.98 \\
\hline misex3 & 49.40 & 3.12 & 0.09 & 52.60 & 1.19 & 44.16 \\
\hline pdc & 356.41 & 20.77 & 0.15 & 377.34 & 2.10 & 179.52 \\
\hline s298 & 80.54 & 4.68 & 0.04 & 85.26 & 0.54 & 156.98 \\
\hline s38417 & 679.76 & 29.44 & 0.08 & 709.28 & 1.13 & 627.73 \\
\hline s38584.1 & 688.35 & 31.85 & 0.09 & 720.29 & 1.24 & 582.35 \\
\hline seq & 69.26 & 4.08 & 0.12 & 73.46 & 1.67 & 43.93 \\
\hline spla & 234.73 & 13.59 & 0.12 & 248.45 & 1.68 & 147.68 \\
\hline tseng & 43.78 & 2.01 & 0.01 & 45.81 & 0.18 & 250.52 \\
\hline Average: & 231.48 & 11.54 & 0.09 & 243.11 & 1.31 & 185.29 \\
\hline
\end{tabular}


Table VI. Dynamic Energy Consumption of MCNC Benchmarks 8bit (100\% Memory)

\begin{tabular}{|c|c|c|c|c|c|c|}
\hline \multirow{2}{*}{$\begin{array}{c}\text { MCNC } \\
\text { Benchmark }\end{array}$} & \multicolumn{4}{|c|}{ Processor Dynamic Energy (nJ) } & \multirow{2}{*}{$\begin{array}{c}\text { FPGA } \\
\text { Dynamic } \\
\text { Energy (nJ) }\end{array}$} & \multirow{2}{*}{$\begin{array}{c}\text { Total CPU/ } \\
\text { Total } \\
\text { FPGA }\end{array}$} \\
\hline & $\begin{array}{c}\text { Instruction } \\
\text { Memory }\end{array}$ & $\begin{array}{c}\text { Data } \\
\text { Memory }\end{array}$ & $\begin{array}{c}\text { Functional } \\
\text { Unit }\end{array}$ & $\begin{array}{l}\text { Total } \\
\text { CPU }\end{array}$ & & \\
\hline alu4 & 56.17 & 7.63 & 0.21 & 64.01 & 2.96 & 21.61 \\
\hline apex2 & 76.99 & 10.37 & 0.28 & 87.65 & 3.98 & 22.01 \\
\hline apex4 & 42.41 & 5.47 & 0.10 & 47.99 & 1.44 & 33.38 \\
\hline bigkey & 75.48 & 11.12 & 0.16 & 86.76 & 2.22 & 39.03 \\
\hline clma & 970.25 & 97.01 & 0.65 & 1067.91 & 9.05 & 117.98 \\
\hline des & 60.04 & 8.49 & 0.29 & 68.81 & 4.05 & 16.99 \\
\hline diffeq & 68.42 & 7.85 & 0.04 & 76.31 & 0.53 & 142.82 \\
\hline dsip & 55.82 & 9.86 & 0.13 & 65.81 & 1.89 & 34.85 \\
\hline elliptic & 326.55 & 30.04 & 0.11 & 356.70 & 1.47 & 242.05 \\
\hline ex1010 & 359.11 & 41.35 & 0.21 & 400.66 & 2.96 & 135.58 \\
\hline ex5p & 32.83 & 4.06 & 0.10 & 36.99 & 1.34 & 27.58 \\
\hline frisk & 303.20 & 26.70 & 0.08 & 329.97 & 1.08 & 305.75 \\
\hline $\operatorname{misex} 3$ & 49.40 & 6.81 & 0.17 & 56.38 & 2.38 & 23.64 \\
\hline pdc & 356.41 & 44.43 & 0.30 & 401.14 & 4.20 & 95.52 \\
\hline s298 & 80.54 & 10.19 & 0.08 & 90.81 & 1.08 & 83.76 \\
\hline s38417 & 679.76 & 62.74 & 0.16 & 742.66 & 2.25 & 329.73 \\
\hline s38584.1 & 688.35 & 67.65 & 0.18 & 756.18 & 2.47 & 305.55 \\
\hline seq & 69.26 & 8.89 & 0.24 & 78.39 & 3.34 & 23.47 \\
\hline spla & 234.73 & 29.25 & 0.24 & 264.22 & 3.37 & 78.48 \\
\hline tseng & 43.78 & 4.41 & 0.03 & 48.22 & 0.37 & 131.92 \\
\hline Average: & 231.48 & 24.72 & 0.19 & 256.38 & 2.62 & 97.76 \\
\hline
\end{tabular}


Table VII. Dynamic Energy Consumption of MCNC Benchmarks 16bit (100\% Memory)

\begin{tabular}{|c|c|c|c|c|c|c|}
\hline \multirow{2}{*}{$\begin{array}{c}\text { MCNC } \\
\text { Benchmark }\end{array}$} & \multicolumn{4}{|c|}{ Processor Dynamic Energy (nJ) } & \multirow{2}{*}{$\begin{array}{c}\text { FPGA } \\
\text { Dynamic } \\
\text { Energy }(\mathbf{n J})\end{array}$} & \multirow{2}{*}{$\begin{array}{c}\text { Total CPU/ } \\
\text { Total } \\
\text { FPGA }\end{array}$} \\
\hline & $\begin{array}{c}\text { Instruction } \\
\text { Memory }\end{array}$ & $\begin{array}{c}\text { Data } \\
\text { Memory }\end{array}$ & $\begin{array}{c}\text { Functional } \\
\text { Unit }\end{array}$ & $\begin{array}{l}\text { Total } \\
C P U\end{array}$ & & \\
\hline alu4 & 56.17 & 18.37 & 0.42 & 74.97 & 5.92 & 12.66 \\
\hline apex2 & 76.99 & 24.91 & 0.57 & 102.48 & 7.98 & 12.84 \\
\hline apex4 & 42.41 & 13.22 & 0.21 & 55.84 & 2.87 & 19.44 \\
\hline bigkey & 75.48 & 26.60 & 0.32 & 102.40 & 4.45 & 22.99 \\
\hline clma & 970.25 & 229.04 & 1.29 & 1200.59 & 18.09 & 66.36 \\
\hline des & 60.04 & 20.42 & 0.58 & 81.04 & 8.09 & 10.01 \\
\hline diffeq & 68.42 & 18.88 & 0.08 & 87.37 & 1.07 & 81.57 \\
\hline dsip & 55.82 & 23.62 & 0.27 & 79.71 & 3.78 & 21.09 \\
\hline elliptic & 326.55 & 71.39 & 0.21 & 398.15 & 2.95 & 135.12 \\
\hline $\operatorname{ex1010}$ & 359.11 & 98.10 & 0.42 & 457.63 & 5.91 & 77.41 \\
\hline $\operatorname{ex5p}$ & 32.83 & 9.85 & 0.19 & 42.88 & 2.67 & 16.04 \\
\hline frisk & 303.20 & 63.63 & 0.15 & 366.98 & 2.16 & 170.02 \\
\hline misex3 & 49.40 & 16.40 & 0.34 & 66.14 & 4.76 & 13.88 \\
\hline pdc & 356.41 & 105.39 & 0.60 & 462.40 & 8.38 & 55.16 \\
\hline s298 & 80.54 & 24.50 & 0.15 & 105.20 & 2.16 & 48.60 \\
\hline s38417 & 679.76 & 148.49 & 0.32 & 828.58 & 4.54 & 182.32 \\
\hline s38584.1 & 688.35 & 159.56 & 0.35 & 848.27 & 4.95 & 171.48 \\
\hline seq & 69.26 & 21.39 & 0.48 & 91.13 & 6.68 & 13.64 \\
\hline spla & 234.73 & 69.71 & 0.48 & 304.93 & 6.73 & 45.32 \\
\hline tseng & 43.78 & 10.65 & 0.05 & 54.48 & 0.73 & 74.47 \\
\hline Average: & 231.48 & 58.71 & 0.37 & 290.56 & 5.24 & 55.40 \\
\hline
\end{tabular}


Table VIII. Dynamic Energy Consumption of MCNC Benchmarks 32bit (100\% Memory)

\begin{tabular}{|c|c|c|c|c|c|c|}
\hline \multirow{2}{*}{$\begin{array}{c}\text { MCNC } \\
\text { Benchmark }\end{array}$} & \multicolumn{4}{|c|}{ Processor Dynamic Energy (nJ) } & \multirow{2}{*}{$\begin{array}{c}\text { FPGA } \\
\text { Dynamic } \\
\text { Energy }(\mathbf{n J})\end{array}$} & \multirow{2}{*}{$\begin{array}{c}\text { Total CPU/ } \\
\text { Total } \\
\text { FPGA }\end{array}$} \\
\hline & $\begin{array}{c}\text { Instruction } \\
\text { Memory }\end{array}$ & $\begin{array}{c}\text { Data } \\
\text { Memory }\end{array}$ & $\begin{array}{c}\text { Functional } \\
\text { Unit }\end{array}$ & $\begin{array}{l}\text { Total } \\
C P U\end{array}$ & & \\
\hline alu4 & 56.17 & 47.44 & 0.85 & 104.46 & 11.85 & 8.82 \\
\hline apex2 & 76.99 & 64.21 & 1.14 & 142.34 & 15.94 & 8.93 \\
\hline apex4 & 42.41 & 34.21 & 0.41 & 77.04 & 5.75 & 13.39 \\
\hline bigkey & 75.48 & 68.49 & 0.63 & 144.60 & 8.89 & 16.27 \\
\hline clma & 970.25 & 583.71 & 2.01 & 1555.97 & 28.12 & 55.34 \\
\hline des & 60.04 & 52.72 & 1.16 & 113.91 & 16.21 & 7.03 \\
\hline diffeq & 68.42 & 48.68 & 0.15 & 117.25 & 2.14 & 54.77 \\
\hline dsip & 55.82 & 60.79 & 0.54 & 117.15 & 7.57 & 15.48 \\
\hline elliptic & 326.55 & 182.72 & 0.42 & 509.69 & 5.90 & 86.43 \\
\hline $\operatorname{ex1010}$ & 359.11 & 250.81 & 0.84 & 610.76 & 11.83 & 51.63 \\
\hline $\operatorname{ex5p}$ & 32.83 & 25.57 & 0.38 & 58.78 & 5.35 & 10.99 \\
\hline frisk & 303.20 & 163.16 & 0.31 & 466.67 & 4.32 & 108.11 \\
\hline misex3 & 49.40 & 42.37 & 0.68 & 92.45 & 9.55 & 9.68 \\
\hline pdc & 356.41 & 269.42 & 1.20 & 627.03 & 16.75 & 37.44 \\
\hline s298 & 80.54 & 63.20 & 0.31 & 144.06 & 4.34 & 33.20 \\
\hline s38417 & 679.76 & 378.78 & 0.64 & 1059.19 & 9.02 & 117.46 \\
\hline s38584.1 & 688.35 & 406.44 & 0.71 & 1095.50 & 9.89 & 110.75 \\
\hline seq & 69.26 & 55.20 & 0.95 & 125.41 & 13.35 & 9.40 \\
\hline spla & 234.73 & 178.76 & 0.96 & 414.45 & 13.46 & 30.79 \\
\hline tseng & 43.78 & 27.55 & 0.10 & 71.43 & 1.46 & 48.84 \\
\hline Average: & 231.48 & 150.21 & 0.72 & 382.41 & 10.08 & 37.93 \\
\hline
\end{tabular}


Table IX. Dynamic Energy Consumption of MCNC Benchmarks 64bit (100\% Memory)

\begin{tabular}{|c|c|c|c|c|c|c|}
\hline \multirow{2}{*}{$\begin{array}{c}\text { MCNC } \\
\text { Benchmark }\end{array}$} & \multicolumn{4}{|c|}{ Processor Dynamic Energy (nJ) } & \multirow{2}{*}{$\begin{array}{c}\text { FPGA } \\
\text { Dynamic } \\
\text { Energy }(\mathbf{n J})\end{array}$} & \multirow{2}{*}{$\begin{array}{c}\text { Total CPU/ } \\
\text { Total } \\
\text { FPGA }\end{array}$} \\
\hline & $\begin{array}{c}\text { Instruction } \\
\text { Memory }\end{array}$ & $\begin{array}{c}\text { Data } \\
\text { Memory }\end{array}$ & $\begin{array}{c}\text { Functional } \\
\text { Unit }\end{array}$ & $\begin{array}{l}\text { Total } \\
C P U\end{array}$ & & \\
\hline alu4 & 56.17 & 127.76 & 1.69 & 185.63 & 23.71 & 7.83 \\
\hline apex2 & 76.99 & 172.78 & 2.28 & 252.05 & 31.90 & 7.90 \\
\hline apex4 & 42.41 & 92.24 & 0.82 & 135.47 & 11.49 & 11.79 \\
\hline bigkey & 75.48 & 184.19 & 1.27 & 260.94 & 17.78 & 14.68 \\
\hline clma & 970.25 & 1560.35 & 4.60 & 2535.20 & 64.41 & 39.36 \\
\hline des & 60.04 & 141.95 & 2.32 & 204.30 & 32.42 & 6.30 \\
\hline diffeq & 68.42 & 131.01 & 0.31 & 199.73 & 4.28 & 46.72 \\
\hline dsip & 55.82 & 163.42 & 1.08 & 220.32 & 15.13 & 14.56 \\
\hline elliptic & 326.55 & 489.64 & 0.26 & 816.46 & 3.70 & 220.71 \\
\hline $\operatorname{ex1010}$ & 359.11 & 671.70 & 1.11 & 1031.92 & 15.57 & 66.26 \\
\hline $\operatorname{ex5p}$ & 32.83 & 69.05 & 0.76 & 102.65 & 10.70 & 9.59 \\
\hline frisk & 303.20 & 437.73 & 0.04 & 740.97 & 0.54 & 1370.15 \\
\hline misex3 & 49.40 & 114.11 & 1.36 & 164.87 & 19.07 & 8.64 \\
\hline pdc & 356.41 & 721.51 & 1.82 & 1079.74 & 25.43 & 42.45 \\
\hline s298 & 80.54 & 170.14 & 0.62 & 251.31 & 8.67 & 28.97 \\
\hline s38417 & 679.76 & 1013.33 & 0.71 & 1693.80 & 9.92 & 170.69 \\
\hline s38584.1 & 688.35 & 1086.28 & 0.84 & 1775.47 & 11.70 & 151.79 \\
\hline seq & 69.26 & 148.64 & 1.91 & 219.81 & 26.73 & 8.22 \\
\hline spla & 234.73 & 479.57 & 1.34 & 715.65 & 18.80 & 38.07 \\
\hline tseng & 43.78 & 74.29 & 0.21 & 118.28 & 2.93 & 40.40 \\
\hline Average: & 231.48 & 402.49 & 1.27 & 635.23 & 17.74 & 35.80 \\
\hline
\end{tabular}


Table X. Dynamic Energy Consumption of MCNC Benchmarks 128bit (100\% Memory)

\begin{tabular}{|c|c|c|c|c|c|c|}
\hline \multirow{2}{*}{$\begin{array}{c}\text { MCNC } \\
\text { Benchmark }\end{array}$} & \multicolumn{4}{|c|}{ Processor Dynamic Energy (nJ) } & \multirow{2}{*}{$\begin{array}{c}\text { FPGA } \\
\text { Dynamic } \\
\text { Energy (nJ) }\end{array}$} & \multirow{2}{*}{$\begin{array}{c}\text { Total CPU/ } \\
\text { Total } \\
\text { FPGA }\end{array}$} \\
\hline & $\begin{array}{c}\text { Instruction } \\
\text { Memory }\end{array}$ & $\begin{array}{c}\text { Data } \\
\text { Memory }\end{array}$ & $\begin{array}{c}\text { Functional } \\
\text { Unit }\end{array}$ & $\begin{array}{l}\text { Total } \\
\text { CPU }\end{array}$ & & \\
\hline alu4 & 56.17 & 352.36 & 3.39 & 411.93 & 47.45 & 8.68 \\
\hline apex2 & 76.99 & 476.17 & 3.98 & 557.15 & 55.76 & 9.99 \\
\hline apex4 & 42.41 & 254.51 & 1.64 & 298.57 & 22.99 & 12.99 \\
\hline bigkey & 75.48 & 506.92 & 1.96 & 584.36 & 27.44 & 21.30 \\
\hline clma & 970.25 & 4285.04 & 9.19 & 5264.49 & 128.68 & 40.91 \\
\hline des & 60.04 & 391.39 & 4.63 & 456.06 & 64.84 & 7.03 \\
\hline diffeq & 68.42 & 361.19 & 0.61 & 430.21 & 8.56 & 50.28 \\
\hline dsip & 55.82 & 450.18 & 2.16 & 508.15 & 30.27 & 16.79 \\
\hline elliptic & 326.55 & 1346.49 & 1.11 & 1674.14 & 15.48 & 108.15 \\
\hline $\operatorname{ex1010}$ & 359.11 & 1846.56 & 2.80 & 2208.46 & 39.17 & 56.39 \\
\hline ex5p & 32.83 & 190.72 & 1.53 & 225.08 & 21.41 & 10.52 \\
\hline frisk & 303.20 & 1204.50 & 0.66 & 1508.35 & 9.18 & 164.37 \\
\hline misex3 & 49.40 & 314.69 & 2.73 & 366.81 & 38.16 & 9.61 \\
\hline pdc & 356.41 & 1983.57 & 4.22 & 2344.20 & 59.10 & 39.67 \\
\hline s298 & 80.54 & 468.97 & 0.66 & 550.17 & 9.23 & 59.62 \\
\hline s38417 & 679.76 & 2785.19 & 1.43 & 3466.38 & 19.99 & 173.39 \\
\hline s38584.1 & 688.35 & 2982.02 & 1.67 & 3672.04 & 23.35 & 157.23 \\
\hline seq & 69.26 & 409.73 & 3.23 & 482.22 & 45.22 & 10.66 \\
\hline spla & 234.73 & 1319.52 & 3.26 & 1557.51 & 45.68 & 34.10 \\
\hline tseng & 43.78 & 204.96 & 0.42 & 249.16 & 5.85 & 42.58 \\
\hline Average: & 231.48 & 1106.73 & 2.56 & 1340.77 & 35.89 & 37.36 \\
\hline
\end{tabular}


Table XI. Dynamic Energy Consumption of MCNC Benchmarks 256bit (100\% Memory)

\begin{tabular}{|c|c|c|c|c|c|c|}
\hline \multirow{2}{*}{$\begin{array}{c}\text { MCNC } \\
\text { Benchmark }\end{array}$} & \multicolumn{4}{|c|}{ Processor Dynamic Energy (nJ) } & \multirow{2}{*}{$\begin{array}{c}\text { FPGA } \\
\text { Dynamic } \\
\text { Energy }(\mathbf{n J})\end{array}$} & \multirow{2}{*}{$\begin{array}{c}\text { Total CPU/ } \\
\text { Total } \\
\text { FPGA }\end{array}$} \\
\hline & $\begin{array}{c}\text { Instruction } \\
\text { Memory }\end{array}$ & $\begin{array}{c}\text { Data } \\
\text { Memory }\end{array}$ & $\begin{array}{c}\text { Functional } \\
\text { Unit }\end{array}$ & $\begin{array}{l}\text { Total } \\
\text { CPU }\end{array}$ & & \\
\hline alu4 & 56.17 & 983.68 & 6.19 & 1046.05 & 86.69 & 12.07 \\
\hline apex2 & 76.99 & 1328.93 & 8.52 & 1414.44 & 119.25 & 11.86 \\
\hline apex4 & 42.41 & 710.86 & 2.71 & 755.97 & 37.88 & 19.96 \\
\hline bigkey & 75.48 & 1412.49 & 4.50 & 1492.47 & 63.01 & 23.69 \\
\hline clma & 970.25 & $\begin{array}{c}11937.6 \\
3\end{array}$ & 17.77 & 12925.66 & 248.82 & 51.95 \\
\hline des & 60.04 & 1092.54 & 8.68 & 1161.25 & 121.47 & 9.56 \\
\hline diffeq & 68.42 & 1008.34 & 0.65 & 1077.41 & 9.04 & 119.16 \\
\hline dsip & 55.82 & 1256.27 & 3.75 & 1315.83 & 52.48 & 25.08 \\
\hline elliptic & 326.55 & 3754.02 & 2.21 & 4082.78 & 30.95 & 131.91 \\
\hline ex1010 & 359.11 & 5147.49 & 5.03 & 5511.63 & 70.49 & 78.19 \\
\hline $\operatorname{ex5p}$ & 32.83 & 532.92 & 2.48 & 568.23 & 34.70 & 16.38 \\
\hline frisk & 303.20 & 3359.29 & 1.31 & 3663.79 & 18.35 & 199.67 \\
\hline misex3 & 49.40 & 878.71 & 4.88 & 932.99 & 68.28 & 13.66 \\
\hline pdc & 356.41 & 5529.24 & 7.86 & 5893.51 & 109.98 & 53.59 \\
\hline s298 & 80.54 & 1309.36 & 1.90 & 1391.80 & 26.61 & 52.31 \\
\hline s38417 & 679.76 & 7760.94 & 2.86 & 8443.56 & 40.06 & 210.79 \\
\hline s38584.1 & 688.35 & 8309.68 & 3.35 & 9001.39 & 46.84 & 192.18 \\
\hline seq & 69.26 & 1143.84 & 7.04 & 1220.13 & 98.50 & 12.39 \\
\hline spla & 234.73 & 3680.12 & 6.52 & 3921.37 & 91.29 & 42.95 \\
\hline tseng & 43.78 & 572.42 & 0.26 & 616.46 & 3.61 & 170.95 \\
\hline Average: & 231.48 & 3085.44 & 4.92 & 3321.84 & 68.91 & 48.20 \\
\hline
\end{tabular}




\subsection{Compressing Memories at 64-bit SIMD}

As discussed in Section 2.3, the energy consumed by a processor can be reduced by increasing the complexity of the functional unit that it contains. This increase in turn reduces both the amount of data and instruction memory that are required to execute the same set of operations. To measure this effect of increasing functional unit complexity on the overall dynamic energy gap between the processor and the FPGA, we measure the overall dynamic energy consumption as a function of reduced memory size. In our measurement, we assume both the instruction memory and data memory are reduced by the same amount and a reduction in memory size leads to a proportional reduction in memory access.

Note that as the complexity of a functional unit increases, the energy per access would increase. The number of accesses, however, will be proportionally reduced. Consequently, in this work we assume the overall total dynamic energy consumed by the functional unit (EFnctional_Unit in Equation (4)) would remain the same as the complexity of the functional unit is increased.

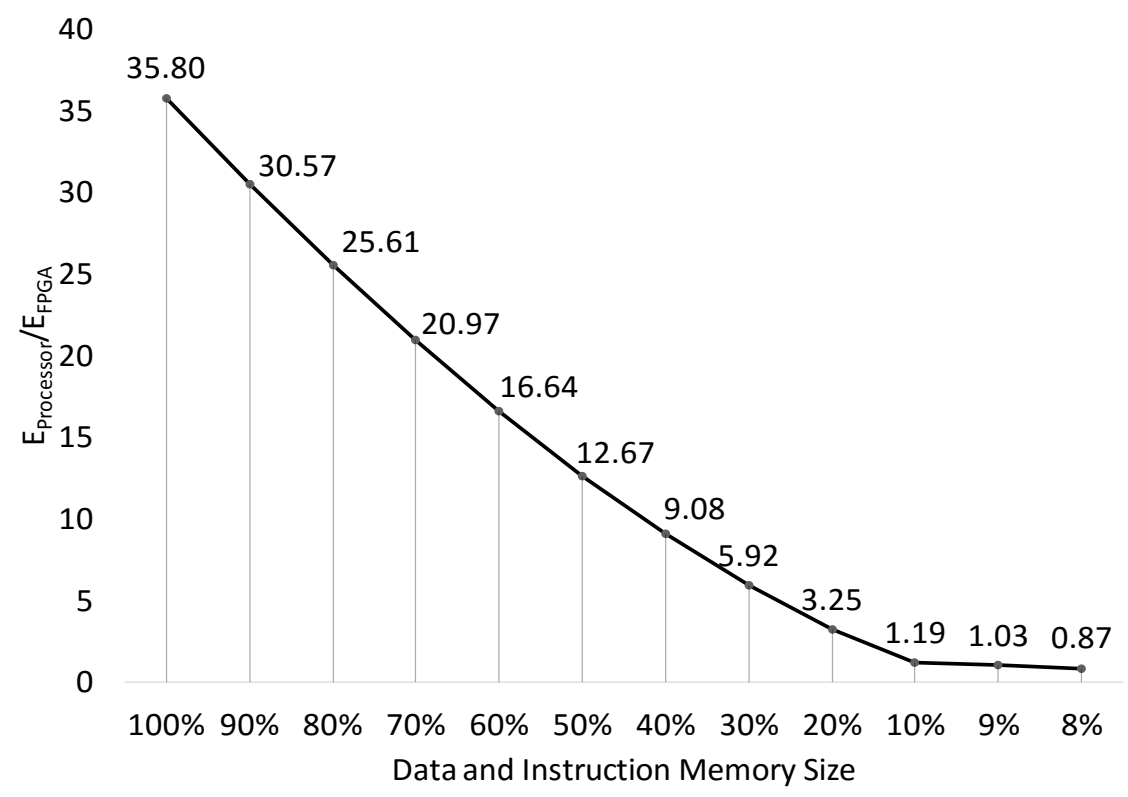

Figure 18. Average Energy Ratio for MCNC Benchmarks at 64bit(s) 
Table XII. Dynamic Energy at 64bit(s) 9\% Memory Size

\begin{tabular}{|c|c|c|c|c|c|c|c|c|}
\hline \multirow{2}{*}{$\begin{array}{c}\text { MCNC } \\
\text { Benchmark }\end{array}$} & \multicolumn{4}{|c|}{ CPU Dynamic Energy (nJ) } & \multicolumn{3}{|c|}{ FPGA Dynamic Energy (nJ) } & \multirow{2}{*}{$\begin{array}{c}\text { Total } \\
\text { CPU/ } \\
\text { Total } \\
\text { FPGA }\end{array}$} \\
\hline & $\begin{array}{c}\text { Instruction } \\
\text { Memory }\end{array}$ & $\begin{array}{c}\text { Data } \\
\text { Memory }\end{array}$ & $\begin{array}{c}\text { Functional } \\
\text { Unit }\end{array}$ & $\begin{array}{l}\text { Total } \\
C P U\end{array}$ & Routing & $\begin{array}{c}\text { LUT } \\
\text { Evaluation }\end{array}$ & $\begin{array}{c}\text { Total } \\
\text { FPGA }\end{array}$ & \\
\hline alu4 & 6.04 & 13.61 & 6.76 & 26.42 & 87.36 & 7.34 & 94.70 & 0.28 \\
\hline apex 2 & 8.31 & 18.33 & 9.11 & 35.75 & 118.43 & 9.06 & 127.49 & 0.28 \\
\hline ex4 & 4.57 & 9.79 & 3.29 & 17.65 & 39.96 & 6.09 & 46.05 & 0.38 \\
\hline bigkey & 8.13 & 19.62 & 5.08 & 32.84 & 62.93 & 8.23 & 71.16 & 0.46 \\
\hline clma & 104.77 & 166.25 & 18.40 & 289.41 & 249.50 & 8.06 & 257.56 & 1.12 \\
\hline des & 6.48 & 15.12 & 9.26 & 30.86 & 122.01 & 7.67 & 129.69 & 0.24 \\
\hline diffeq & 7.38 & 13.96 & 1.22 & 22.55 & 9.90 & 7.21 & 17.10 & 1.32 \\
\hline dsip & 6.02 & 17.37 & 4.32 & 27.71 & 53.82 & 6.61 & 60.43 & 0.46 \\
\hline elliptic & 35.26 & 52.07 & 1.06 & 88.39 & 29.77 & 15.00 & 14.77 & 5.98 \\
\hline ex1010 & 3.53 & 7.28 & 3.06 & 13.87 & 37.67 & 5.13 & 42.81 & 0.32 \\
\hline ex5p & 38.74 & 71.51 & 4.44 & 114.69 & 72.31 & 10.19 & 62.12 & 1.85 \\
\hline frisk & 32.72 & 46.63 & 0.15 & 79.51 & 17.46 & 15.30 & 2.16 & 36.83 \\
\hline $\operatorname{misex} 3$ & 5.32 & 12.15 & 5.45 & 22.92 & 69.58 & 6.74 & 76.32 & 0.30 \\
\hline pdc & 38.46 & 76.82 & 7.28 & 122.56 & 112.24 & 10.30 & 101.94 & 1.20 \\
\hline s298 & 8.68 & 18.09 & 2.48 & 29.25 & 25.40 & 9.31 & 34.71 & 0.84 \\
\hline s38417 & 73.37 & 107.92 & 2.88 & 184.17 & 43.25 & 2.97 & 40.29 & 4.57 \\
\hline s38584.1 & 74.32 & 115.70 & 3.34 & 193.36 & 48.78 & 2.07 & 46.71 & 4.14 \\
\hline seq & 7.47 & 15.80 & 7.63 & 30.91 & 98.44 & 8.44 & 106.89 & 0.29 \\
\hline spla & 25.35 & 51.09 & 5.36 & 81.80 & 89.66 & 14.57 & 75.09 & 1.09 \\
\hline tseng & 4.73 & 7.85 & 0.83 & 13.41 & 6.64 & 5.05 & 11.69 & 1.15 \\
\hline Average: & 24.98 & 42.85 & 5.07 & 72.90 & 69.76 & 8.27 & 70.98 & 1.03 \\
\hline
\end{tabular}


Figure 18 shows the ratio between the dynamic energy consumption of the processor ( $\left.E_{\text {processor }}\right)$ and the dynamic energy consumption of the FPGA $\left(\mathrm{E}_{\mathrm{FPGA}}\right)$ as the instruction and data memory sizes are reduced. As shown, when the memory size (and access) is reduced to around $9 \%$ of the memory required for a processor that employs 64-bit wide 4-LUT-based functional unit, the processor consumes the same amount of dynamic energy as the 4-LUT-based FPGA.

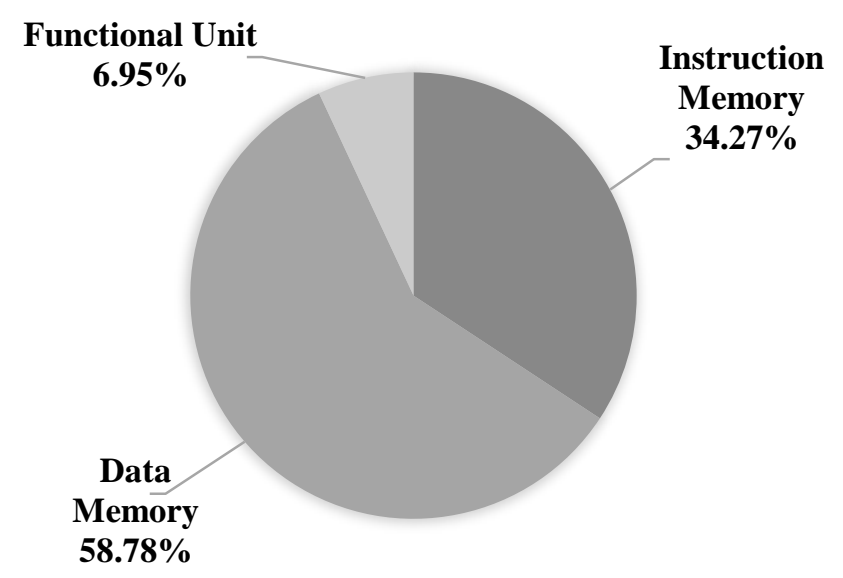

Figure 19. Energy Distribution at 64bit (9\% Memory)

The detailed dynamic energy consumption at the 9\% memory sizes is shown in Table XII for each benchmark circuit. As shown the energy consumption ratio between the processor and FPGA also varies as the benchmark set is varied. In particular, for the largest 10 benchmarks, on average the processor consumes $1.21 \mathrm{x}$ the energy of the 4-LUT-based FPGA at 9\% memory size. For the smallest 10 benchmarks, on the other hand, the processor consumes only $0.36 \mathrm{x}$ the energy of the 4-LUT-based FPGA at the same memory size - i.e. the processor is already $3 \times$ times more dynamic energy efficient than the FPGA for the smaller benchmarks.

Finally, Figure 19 provides the energy distribution among the functional unit and the instruction and data memory. As shown, as the size of the data and instruction memory is reduced, the functional unit consumes an increased proportion of the total dynamic energy. At 9\% memory size, 
$6.95 \%$ of the total dynamic energy is consumed by the functional unit while the instruction and data memory consume $34.27 \%$ and $58.78 \%$ of the total dynamic energy respectively. Figure 20 provides the energies consumed by both CPU and FPGA at 9\% memory for 64-bit wide data path.

MCNC Benchmark Energy Consumption (nJ)

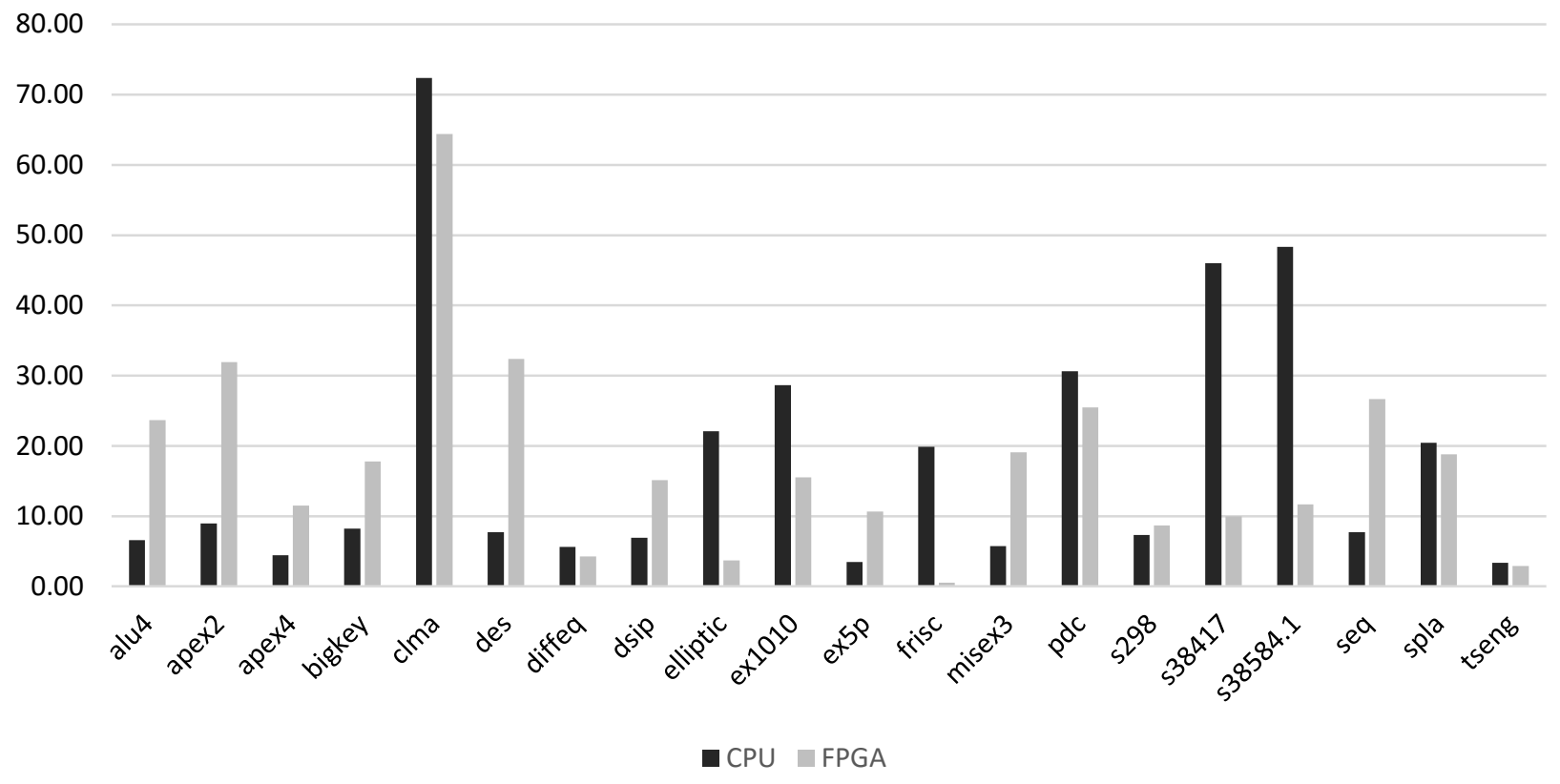

Figure 20. Dynamic Energy Consumption at 64bit(s) $9 \%$ Memory 


\section{Chapter 5}

\section{Conclusion and Future work}

\subsection{Conclusion}

In this work, we first measured the dynamic energy difference between the 4-LUT FPGA and a 4LUT-based sequential processor, by implementing the MCNC benchmarks on both processing platforms. A single 4-LUT processor was found to be much more inefficient in consuming dynamic energy than a 4-LUT FPGA. We optimized the processor for dynamic energy by increasing the number of 4-LUTs in its functional units and then performed the same measurements for our benchmarks. We showed that the average dynamic energy ratio between the processor and the FPGA is at a minimum when the processor contains 64 4-LUTs as its functional unit. Adding more 4-LUTs increases this ratio.

We then measured dynamic energy gap between the processor and the CPU as the complexity of the functional unit is increased. We found that for the MCNC benchmarks, on average, if a processor with ASIC-based functional unit can perform the same computation while consuming less than $9 \%$ of the instruction and data as is required by the same processor with 64-bit wide 4LUT-based functional unit, it can be more efficient in dynamic energy than a 4-LUT-based FPGA. Our results show that the dynamic energy consumption of processors and FPGAs is not only a function of their sequential and parallel execution models but also a function of the complexity of the functional units/accelerators that they contain. 


\subsection{Future work}

Future work, can be performed by adding static energy consumption estimations for the system. Also memory models can be improved by adding gate capacitances as well which are ignored here to keep the model simple and only wire capacitances are considered. Our simulator can be grouped as a part of the CAD tool chain for implementing future designs on complex System-On-Chip (SoC), containing both sequential and parallel processing capabilities on same Integrated Circuit (IC). After some further exploration this tool can make decisions at the compile-time to distribute computations between the parallel and sequential processing elements available, based on the dynamic energy requirements.

Since it is much easier to increase the complexity of a functional unit in the sequential execution model of a processor than the parallel execution model employed by an FPGA, it is highly important for the FPGA research community to continue to increase the complexity of the hard blocks that FPGAs employ while maintaining the utilization of these blocks under the parallel execution model in order for FPGAs to maintain their dynamic energy advantage over the processors. 


\section{Appendix A}

\section{Source Code (.c and .h Files)}

Source code for simulation is provided in this appendix, we have reused some code from T-Vpack to read the MCNC Benchmarks in BLIF format in file 'read_blif.c' and some utility functions in file 'util.c'. Some part of 'main.c' is also reused from T-Vpack, rest of the code in files 'graph.c', 'simulation.c' and 'read_route.c' is completely new code. This section provides the code for all these file, which includes the reused and new code both in the order we used for our simulation.

Header files and makefiles to compile this code on Linux operating system are provided after the c-files. In the end of this appendix, I have provided the BLIF files used for the example in chapter 2.

Following is the list of all files:

c-files:

1. main.c

2. read_blif.c

3. util.c

4. graph.c

5. simulation.c

6. read_route.c

h-files:

1. globals.h

2. graph.h

3. read_blif.h

4. read_route.h

5. simulation.h

6. util.h

7. vpack.h

blif files:

1. 8_bit_compressor_tree

2. 8_Full_Adders

3. Full_Adder 


\section{c-files:}

\section{main.c:}

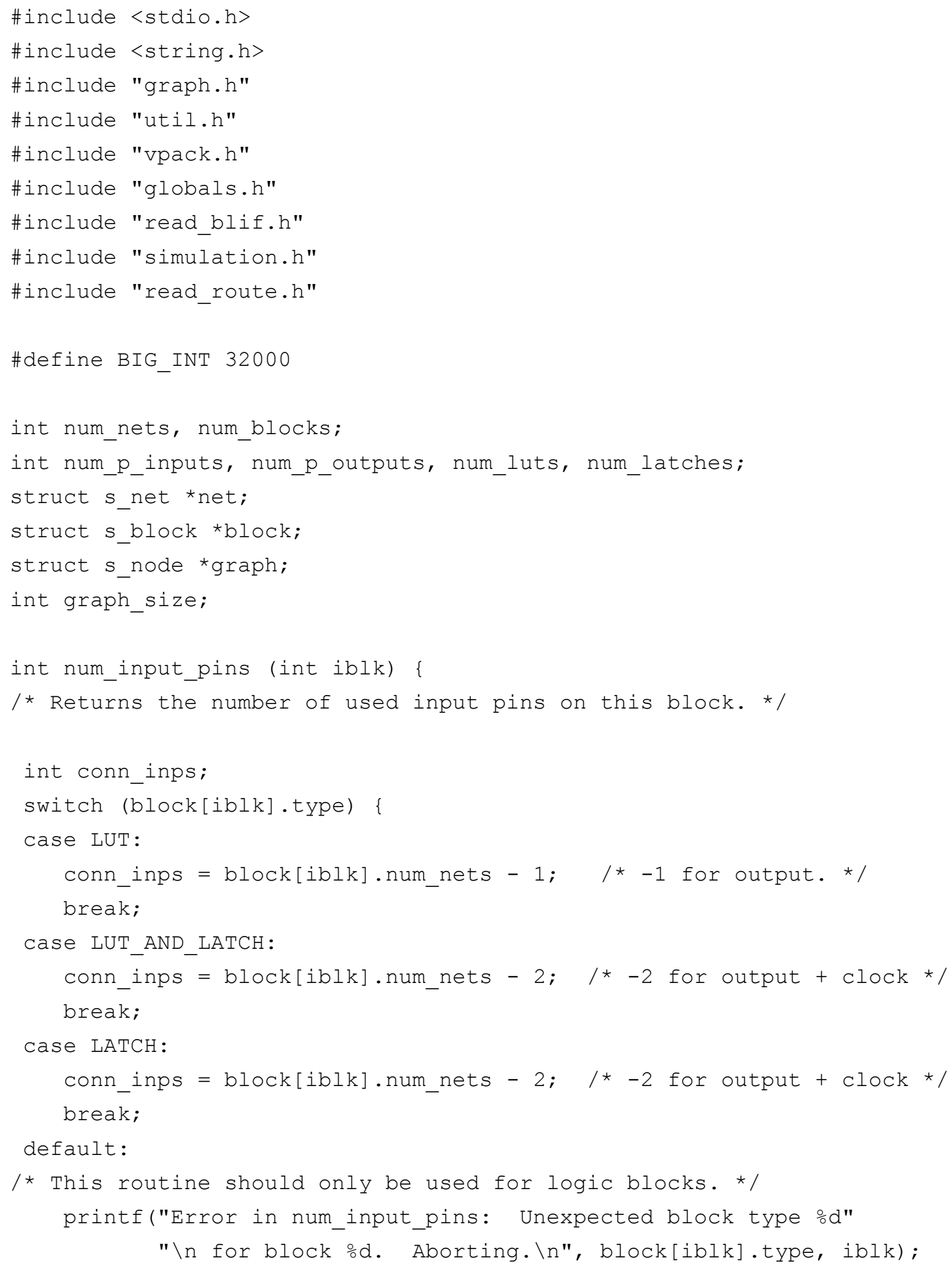




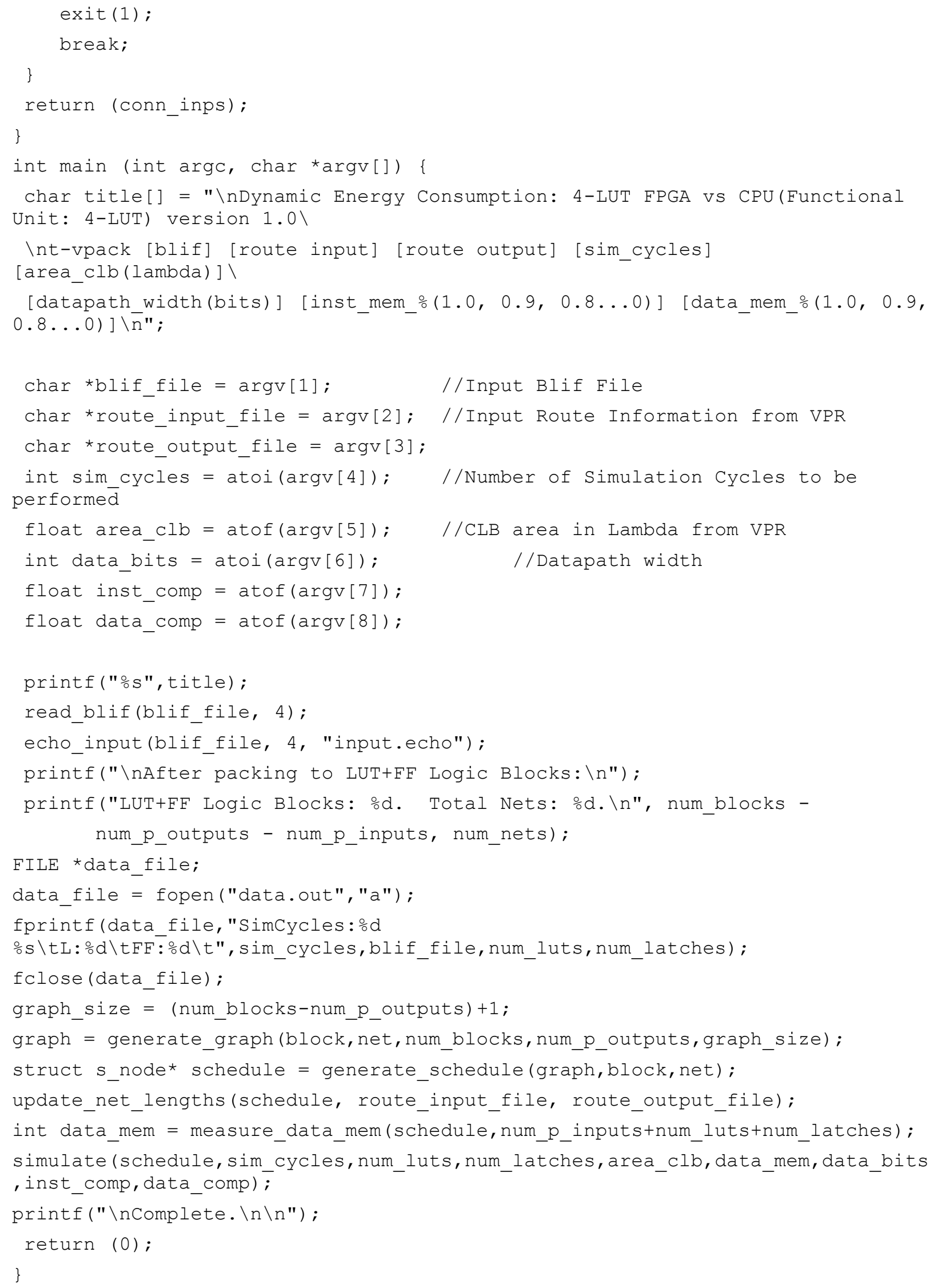




\section{read_blif.c:}

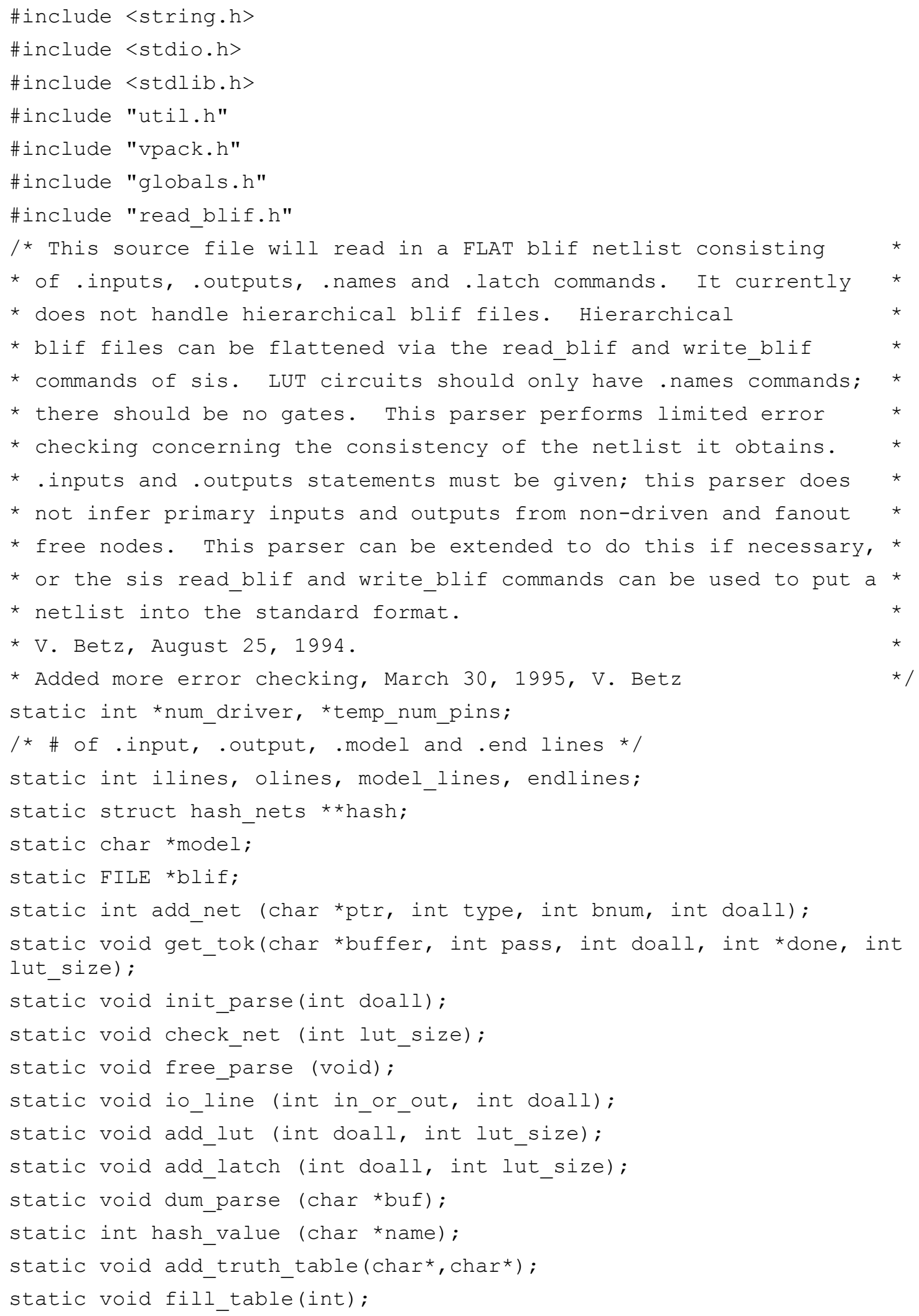




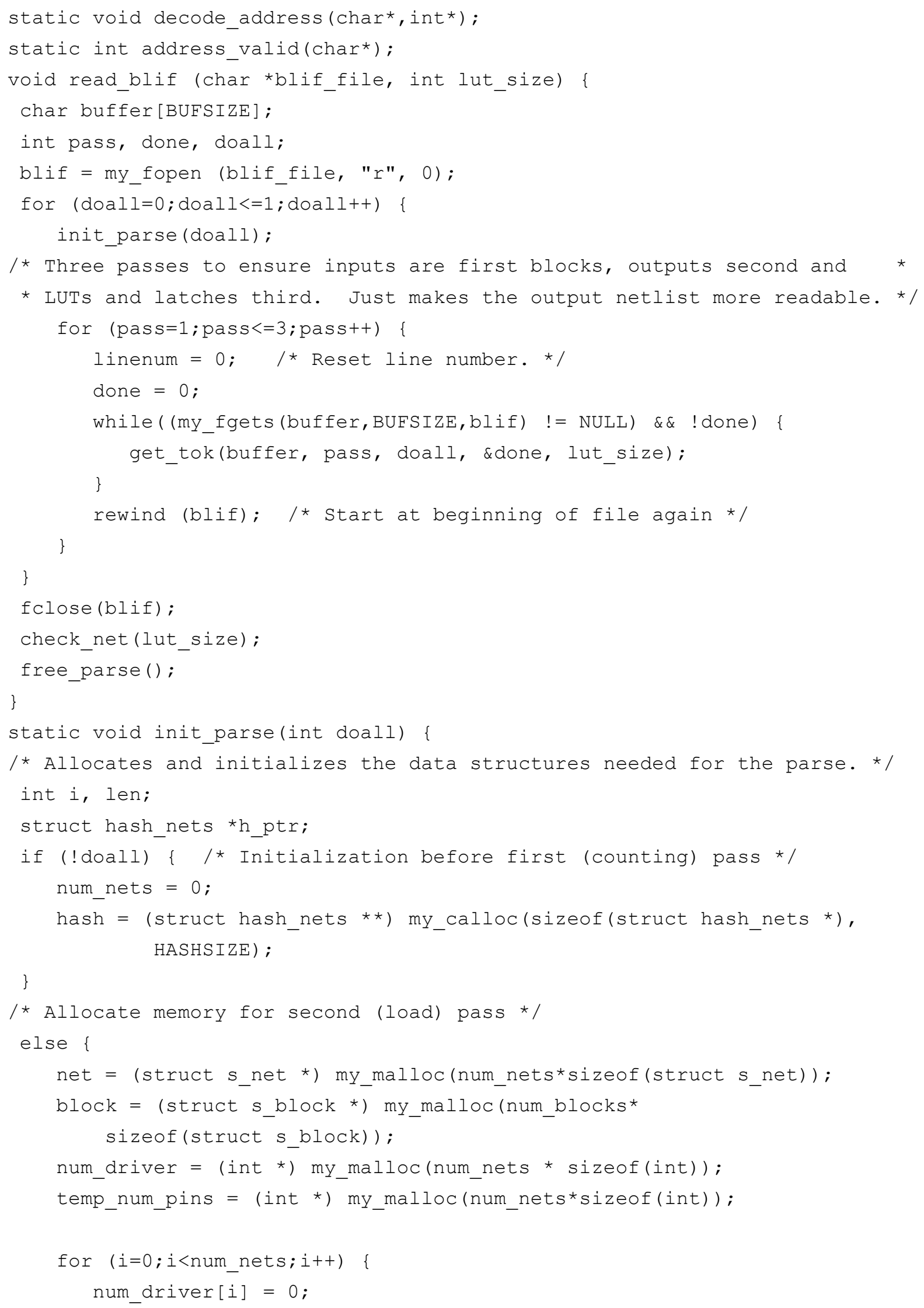




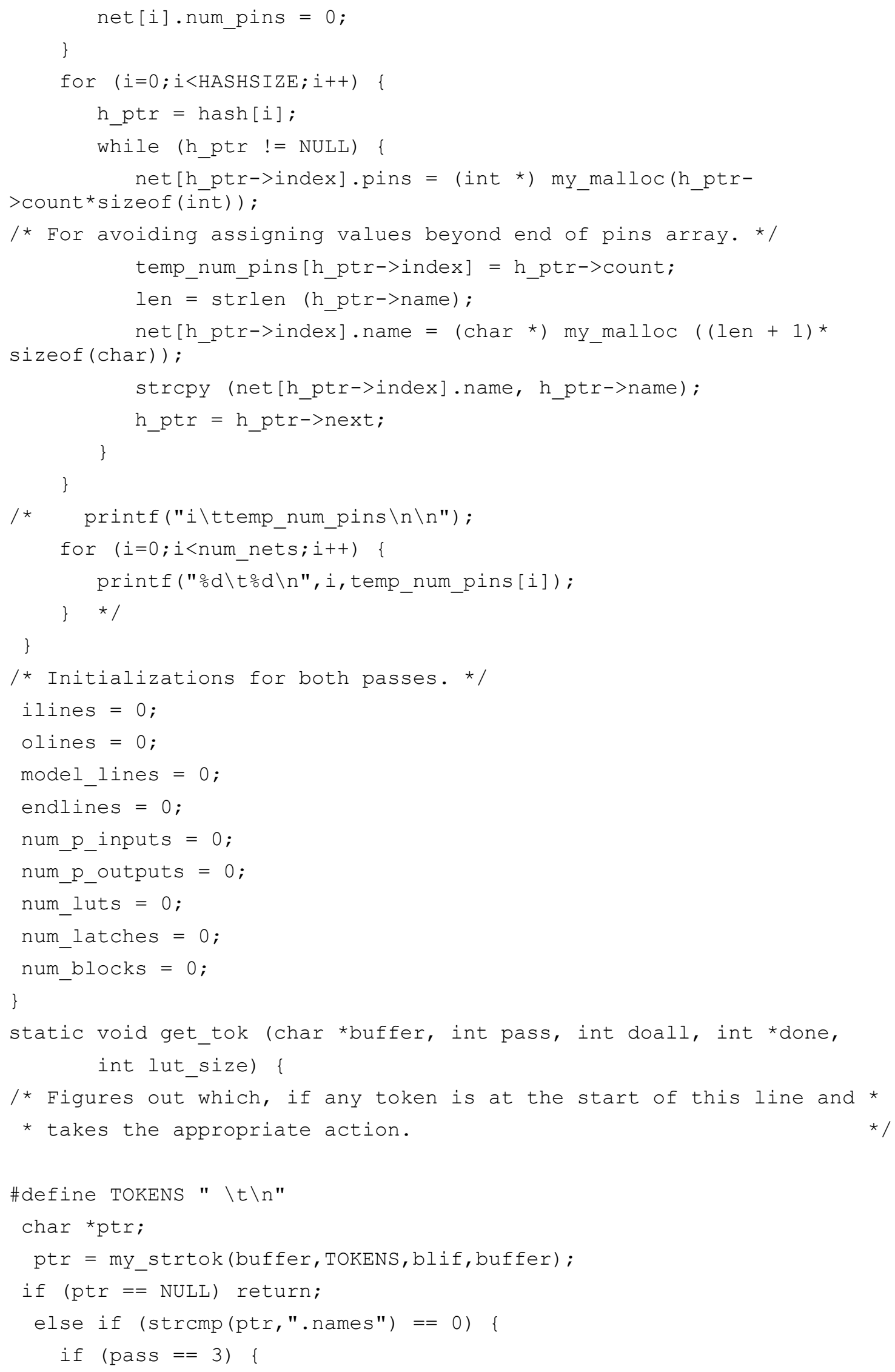




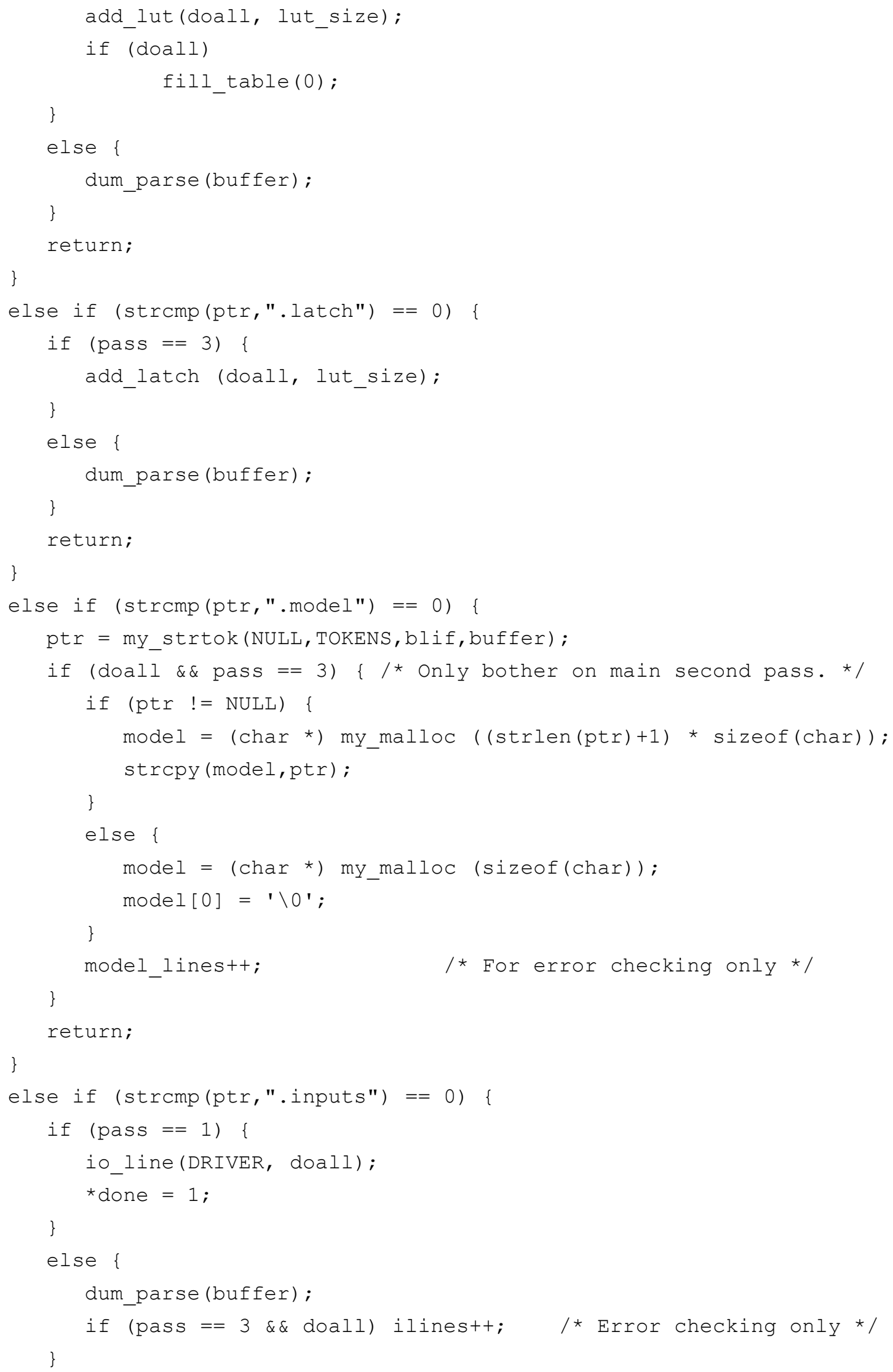




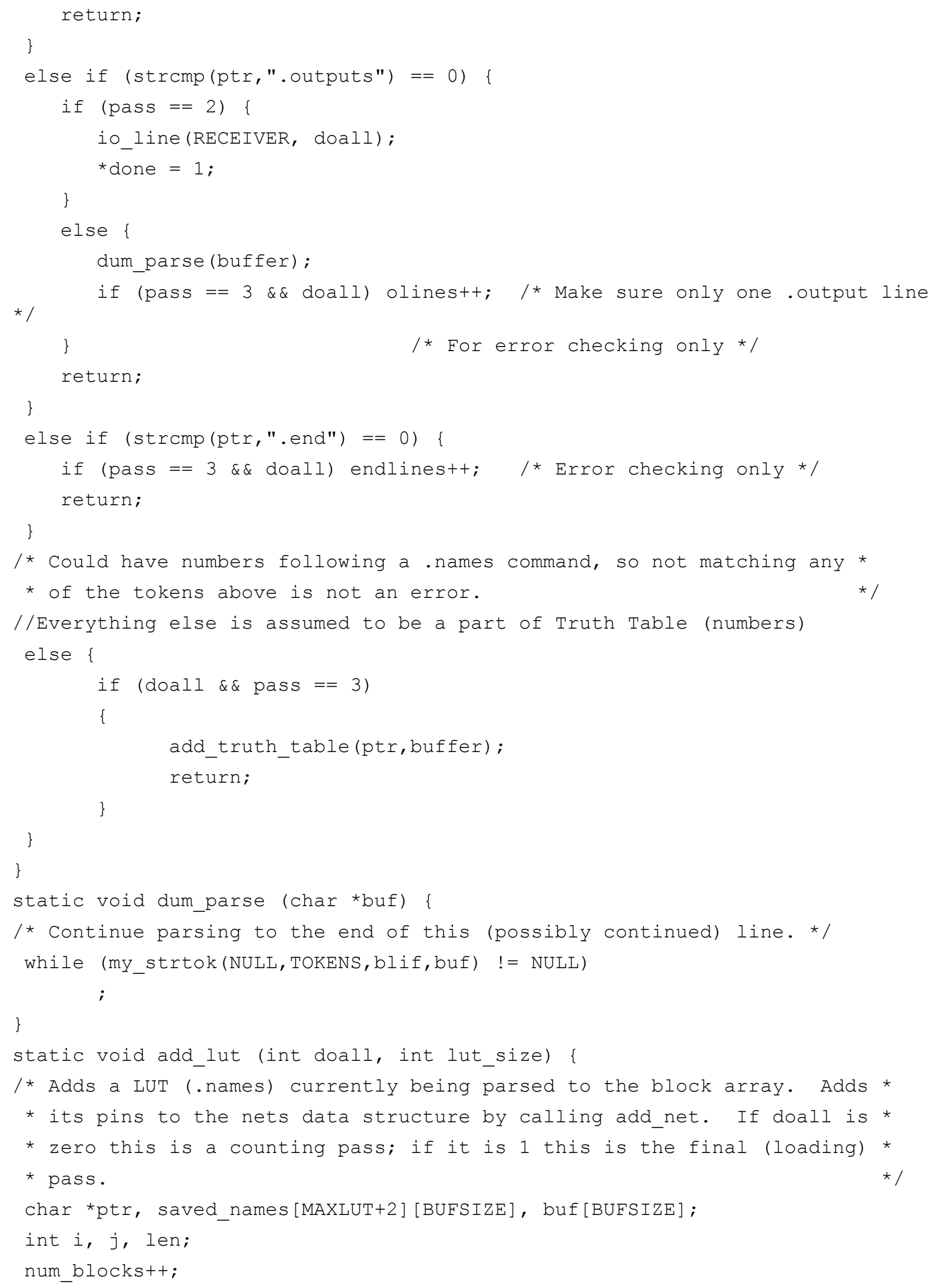




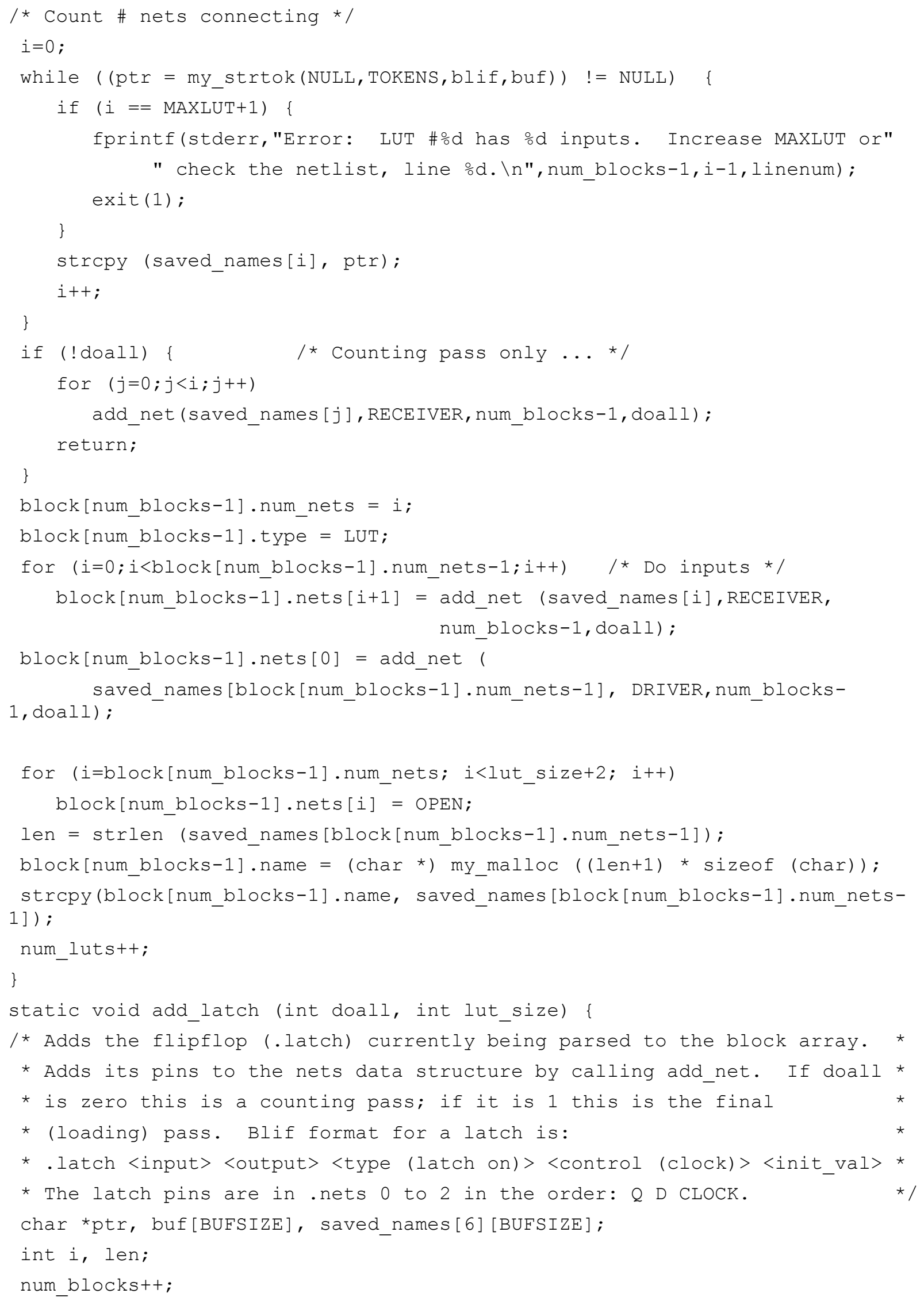


/* Count \# parameters, making sure we don't go over 6 (avoids memory corr.) $\star /$

/* Note that we can't rely on the tokens being around unless we copy them. */

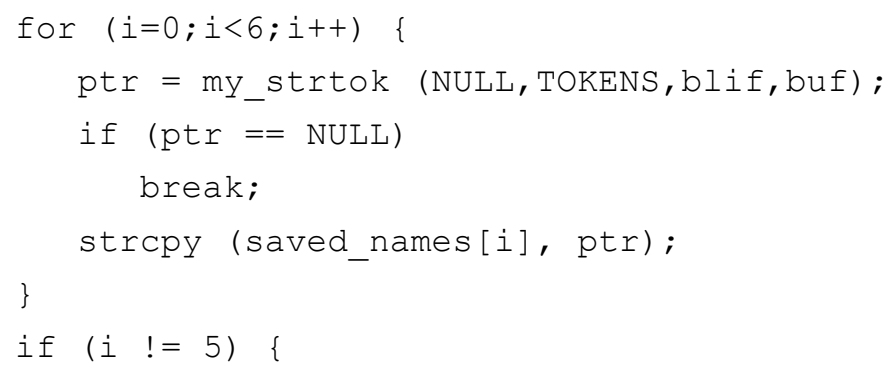




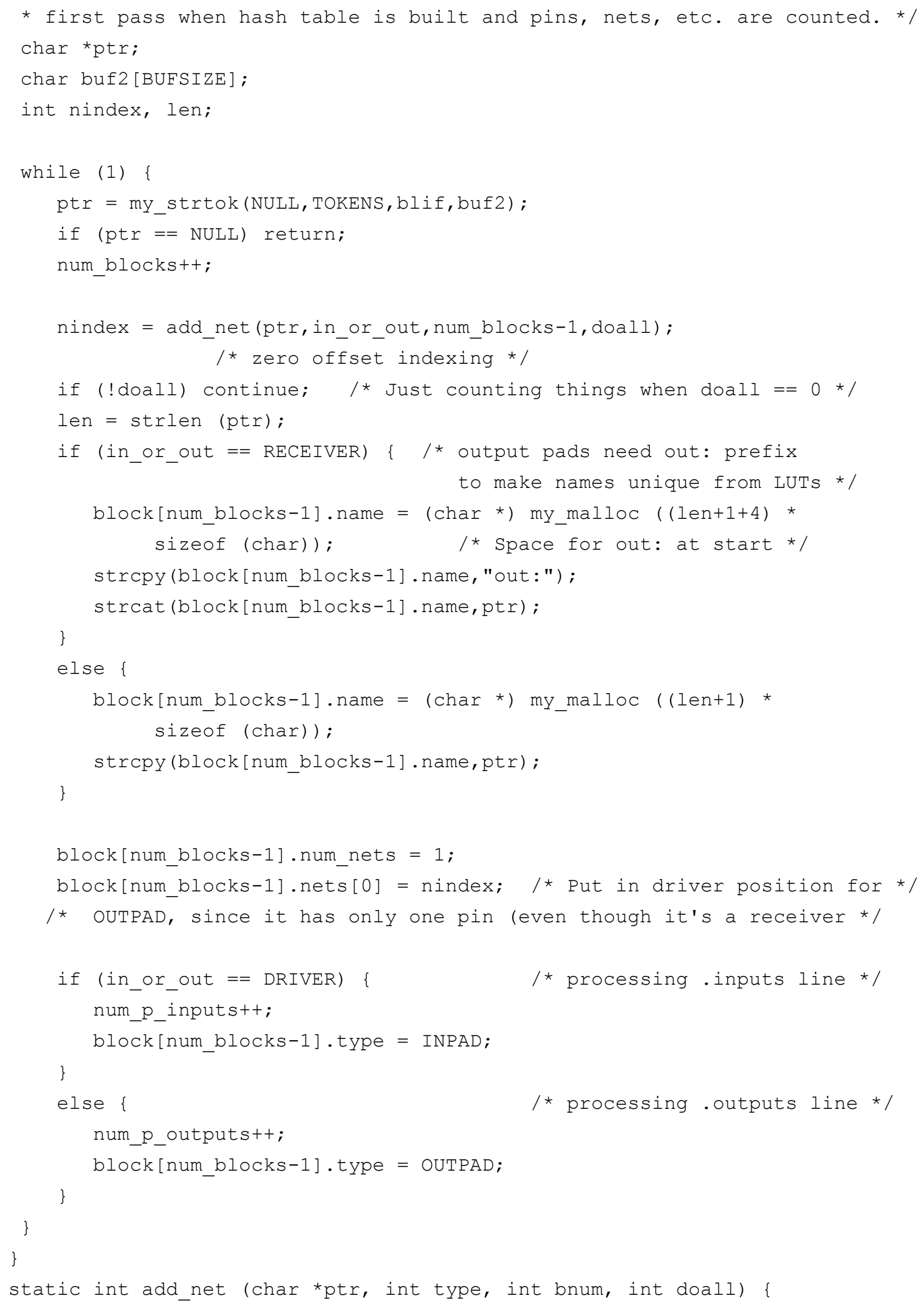




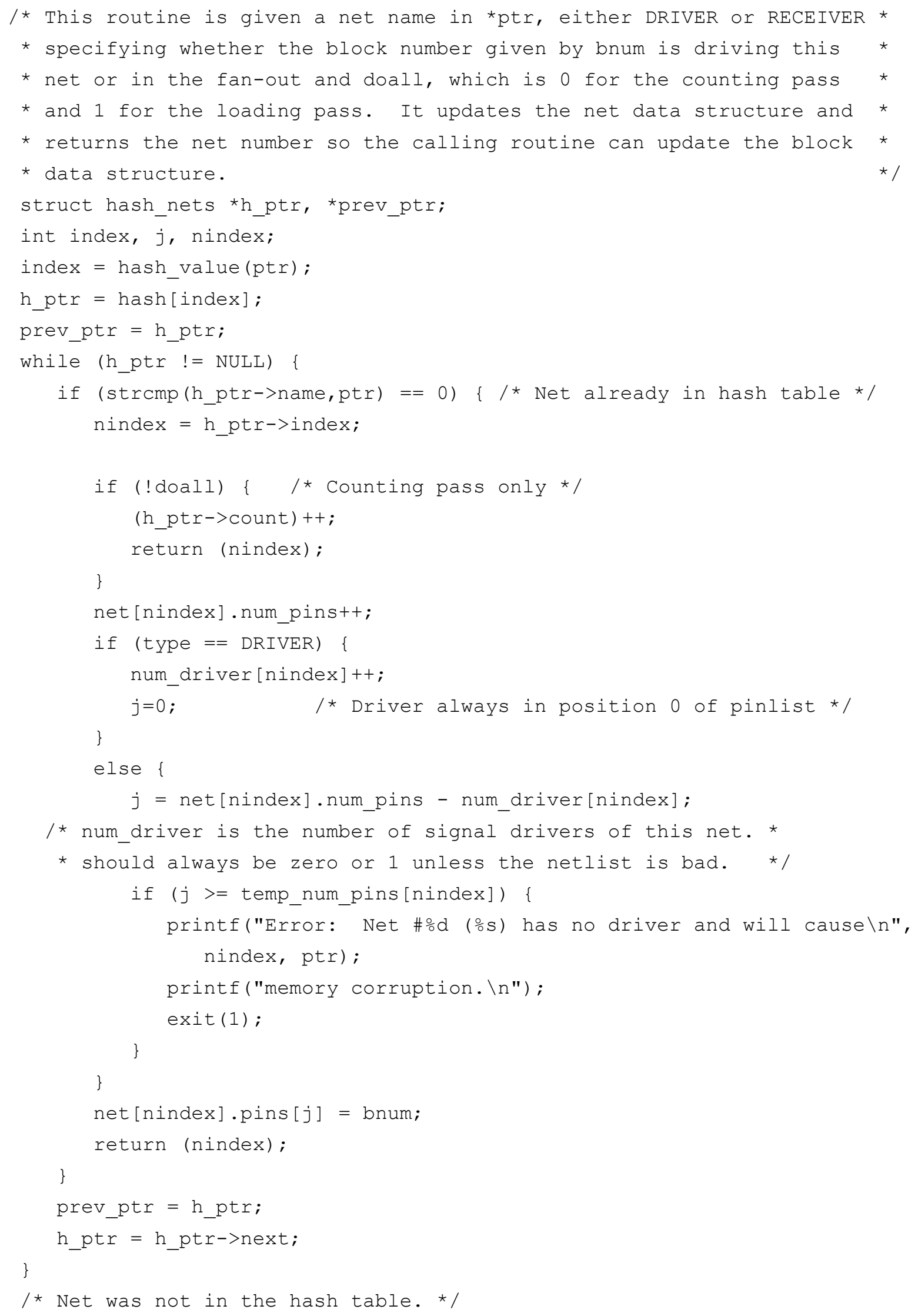




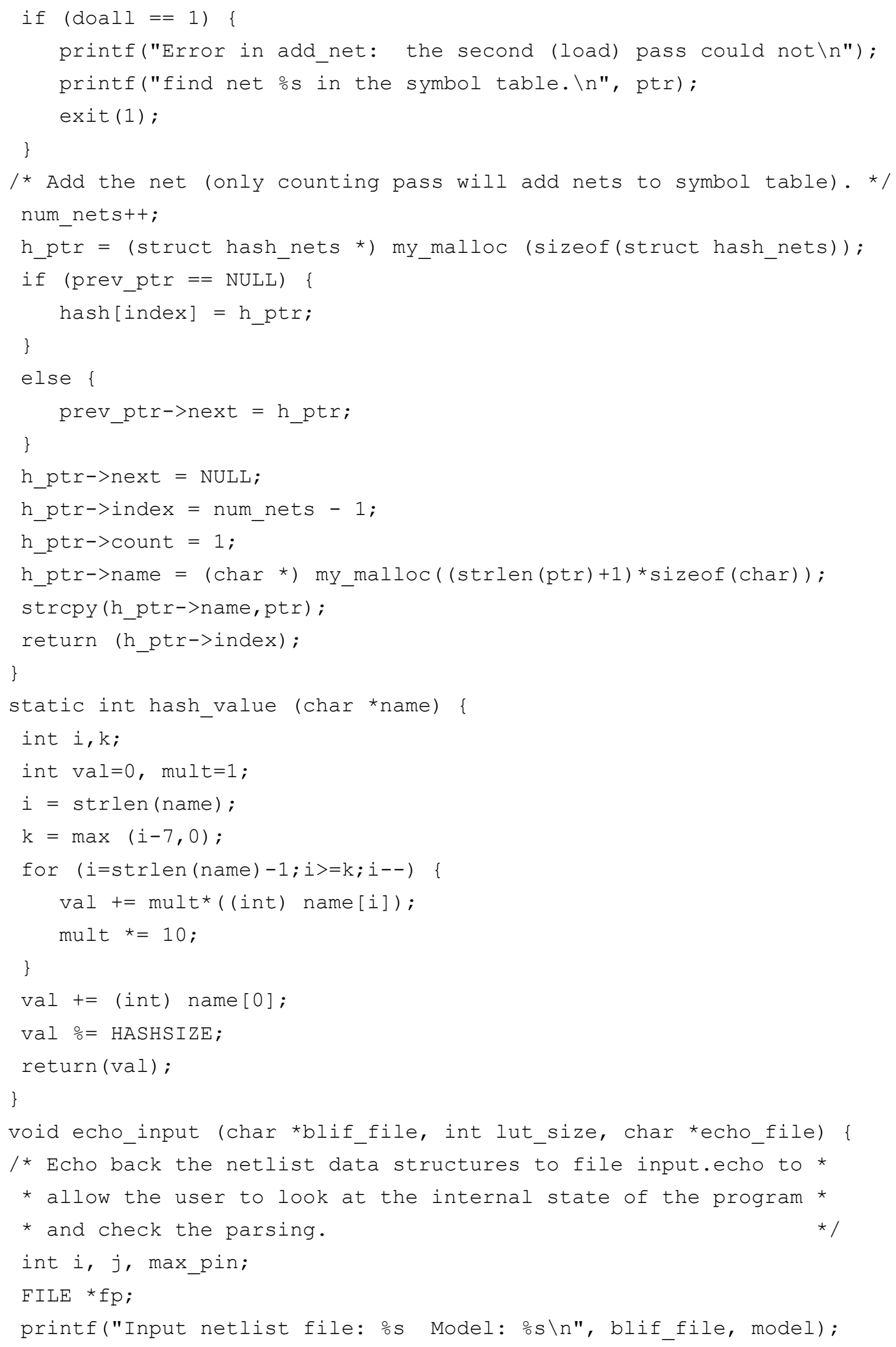




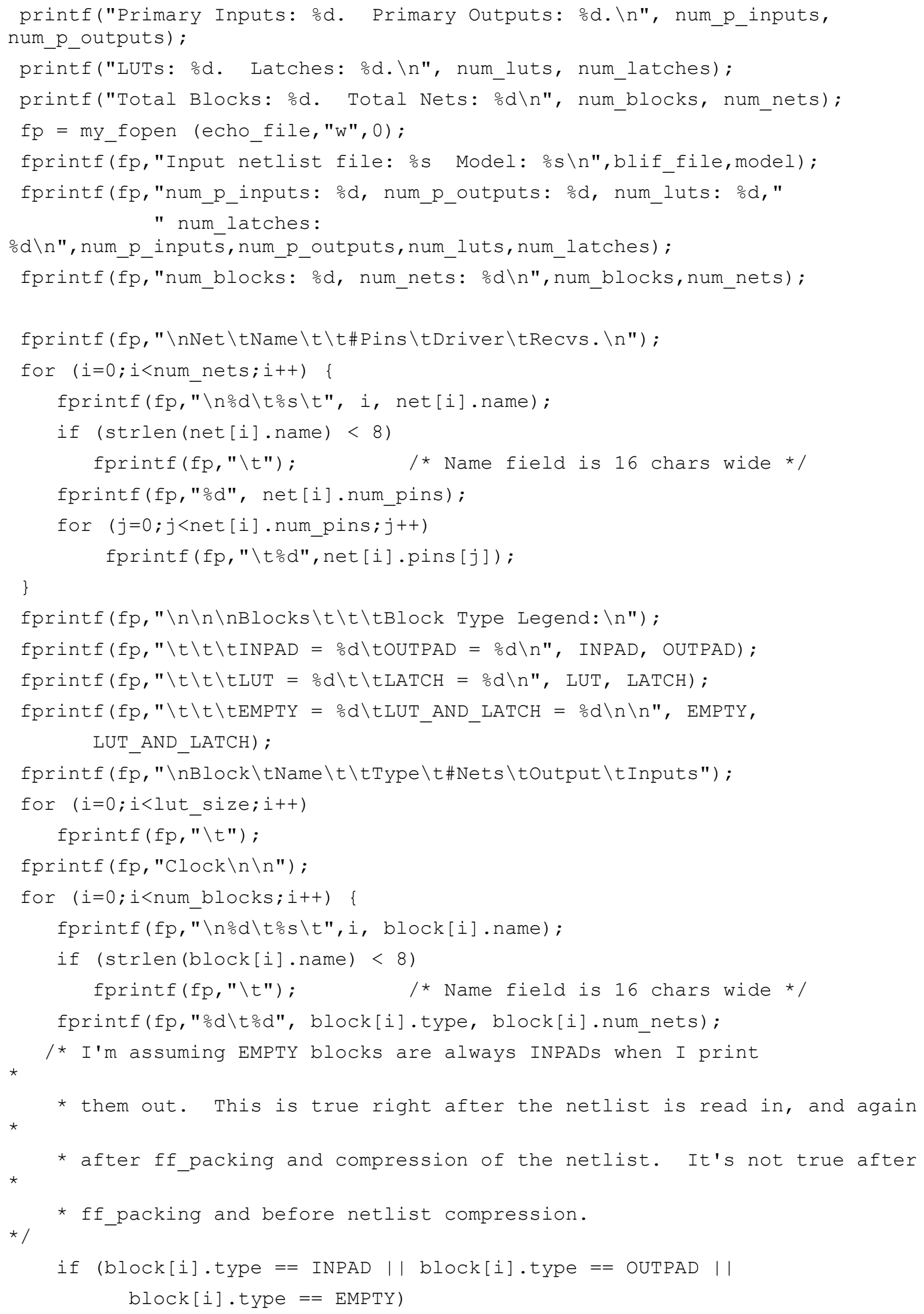




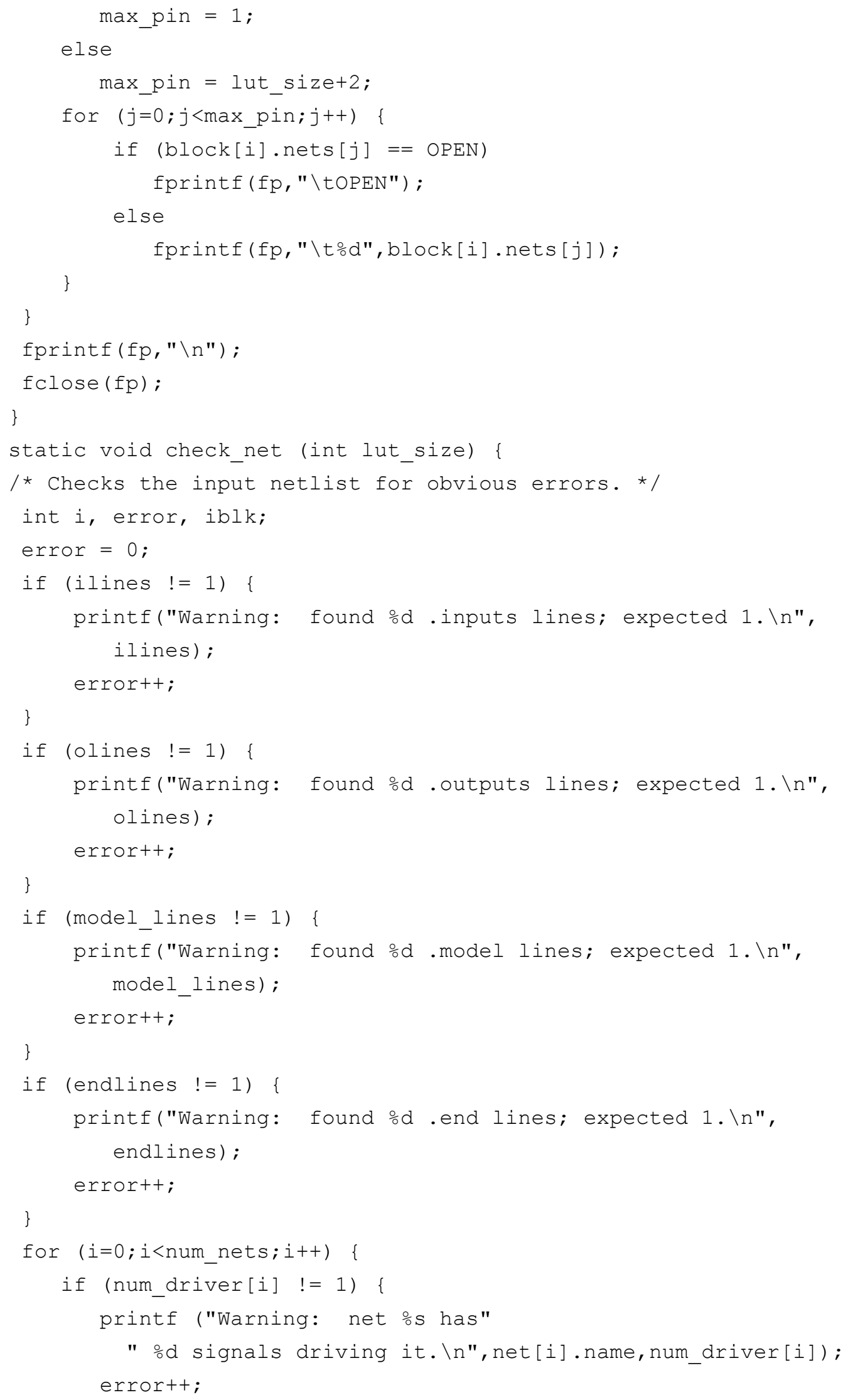




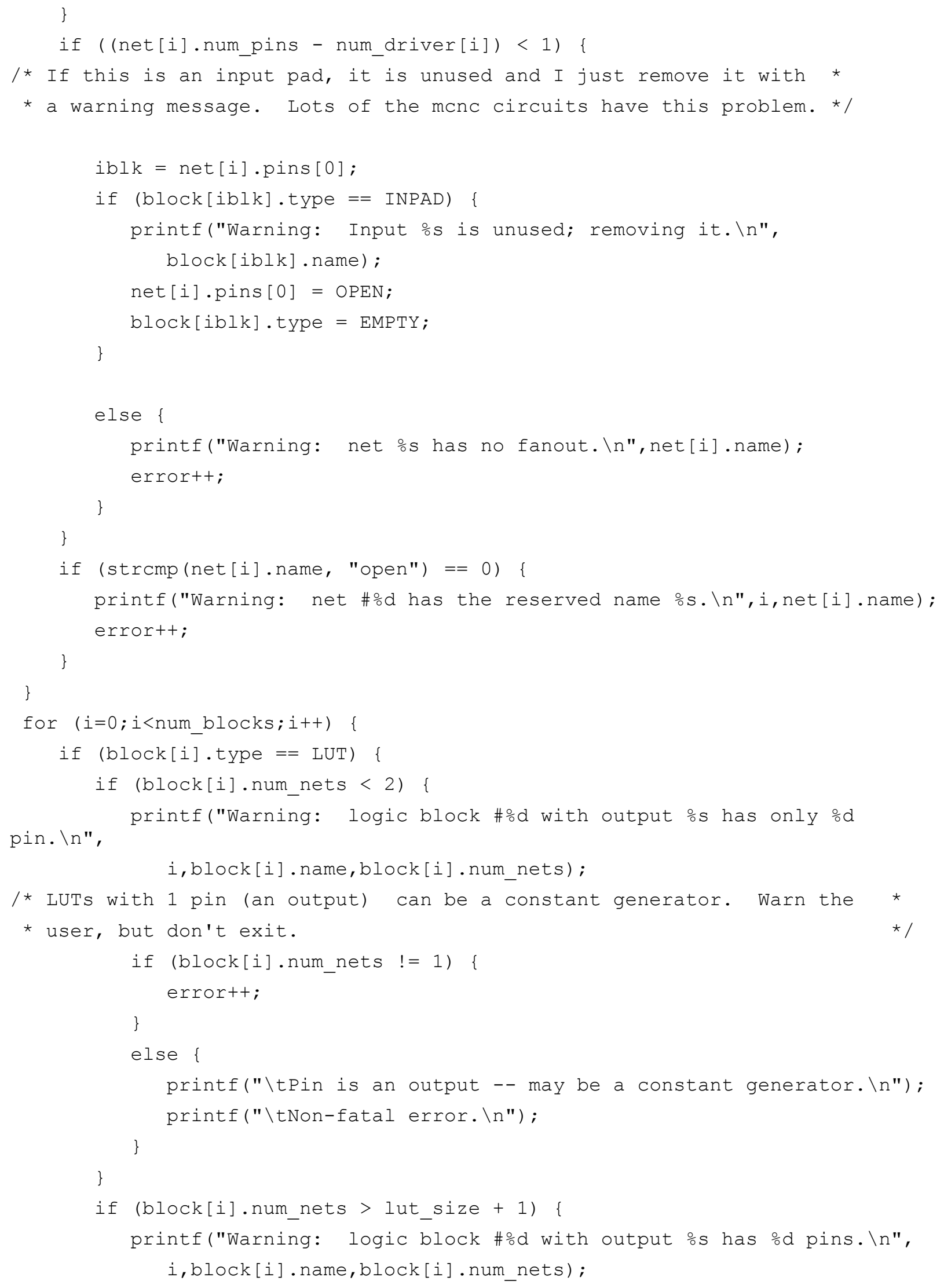




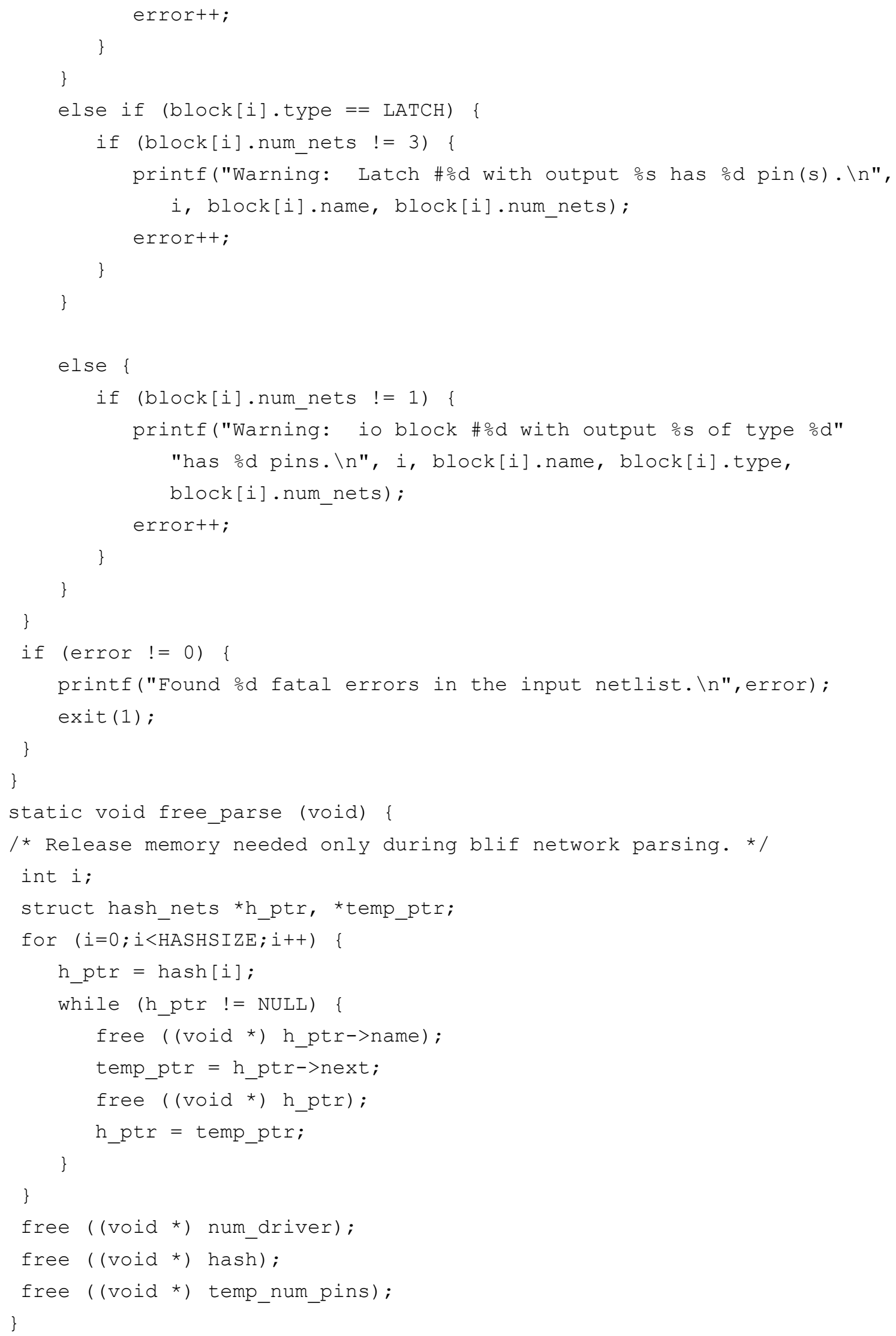


//Adding Truth Table Function

static void add_truth_table (char *input, char *buffer)

\{

char *output;

if ( output $=$ my_strtok (NULL, TOKENS, blif,buffer) $)==$ NULL) \{

output = input;

if (strcmp (output," $1 "$ ) = = 0)

fill_table(1); //Char to Int

else if(strcmp (output,"0") ==0)

fill_table (0);

\}else //If there are more than one values in truth table

\{

//Decode charaters (Binary) into dec address (array Index)

int address [16];

int i;

for $(i=0 ; i<16$; $i++)$

address $[i]=0$;

decode_address (input, address) ;

for $(i=0 ; i<16 ; i++)$

\{

if (address $[i]==1$ )

\{

if (strcmp (output,"1") == 0 )

block[num_blocks-1].truth_table[i] = 1 ;

else if (strcmp (output,"0") ==0)

block[num_blocks-1].truth_table [i] = 0 ;

\}

\}

\}

return;

\}

//Fill the whole truth table with input

static void fill_table (int value)

\{

int $\mathrm{n}=0$;

for $(\mathrm{n}=0 ; \mathrm{n}<16 ; \mathrm{n}++)$

block[num_blocks-1].truth_table $[\mathrm{n}]=$ value;

return;

\}

//Decode Address Function

static void decode_address (char *input, int *address)

\{ 


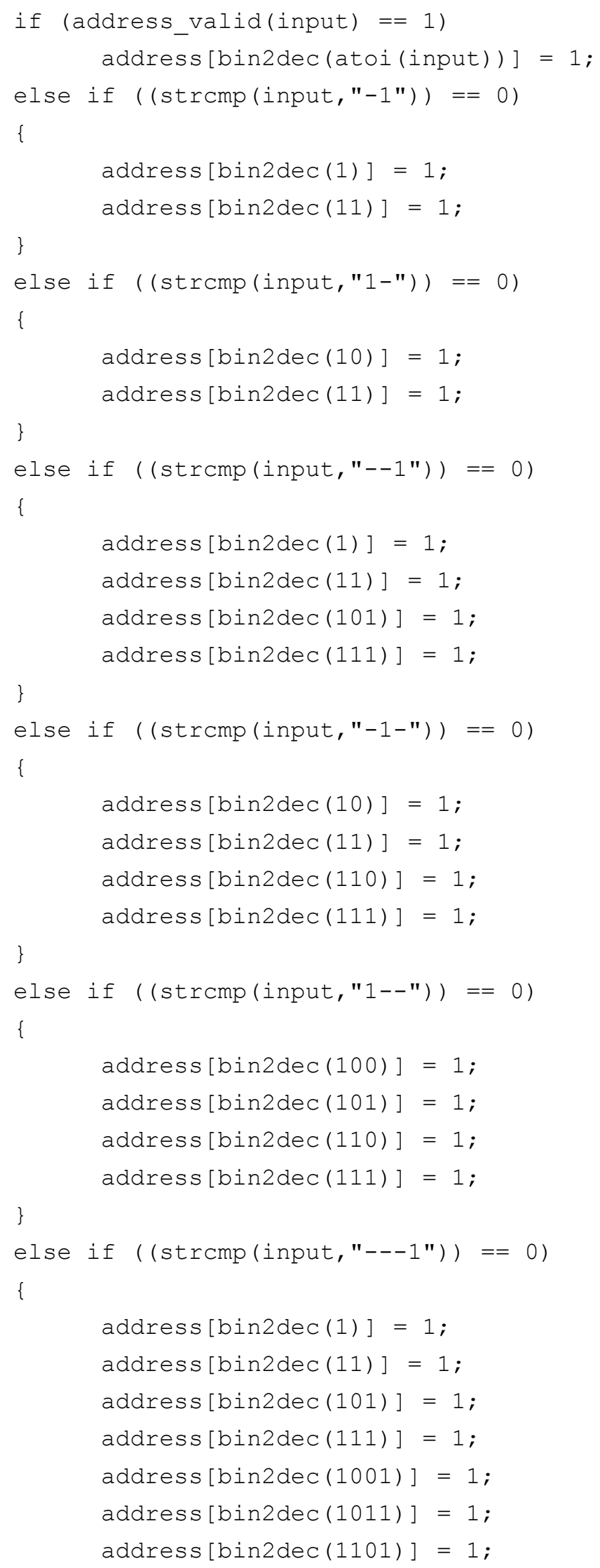




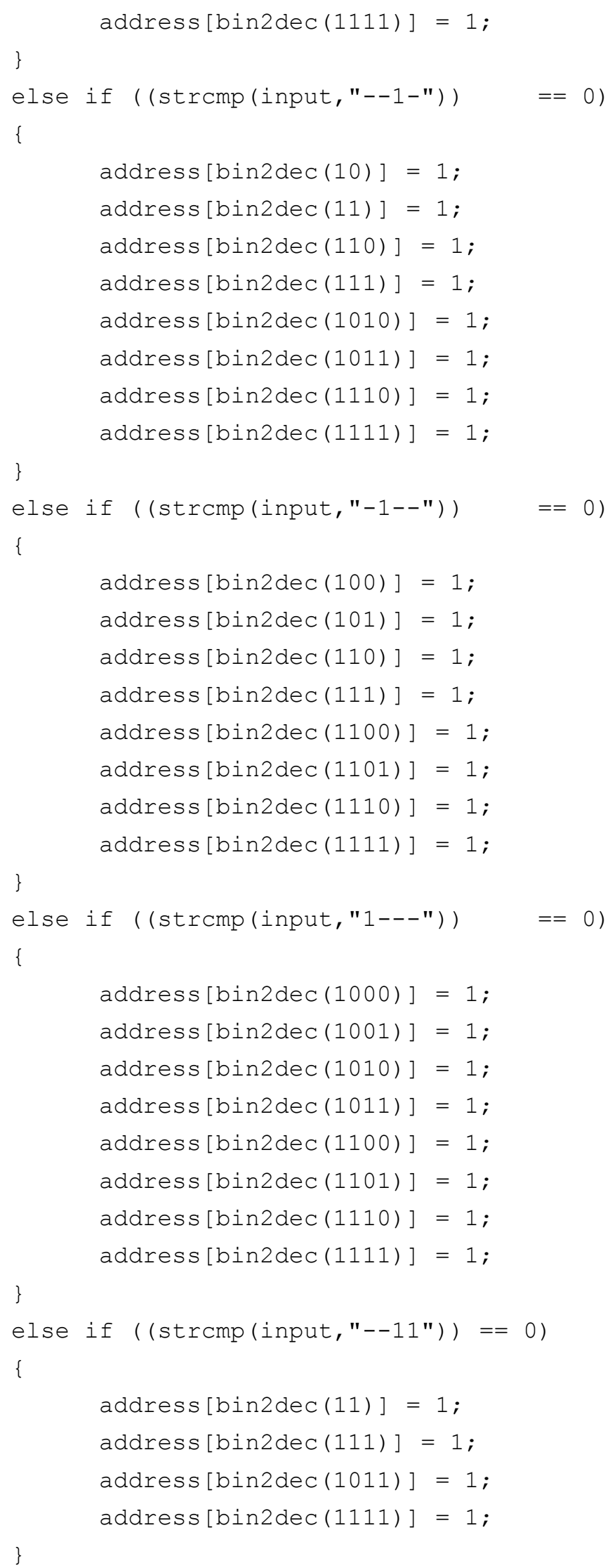




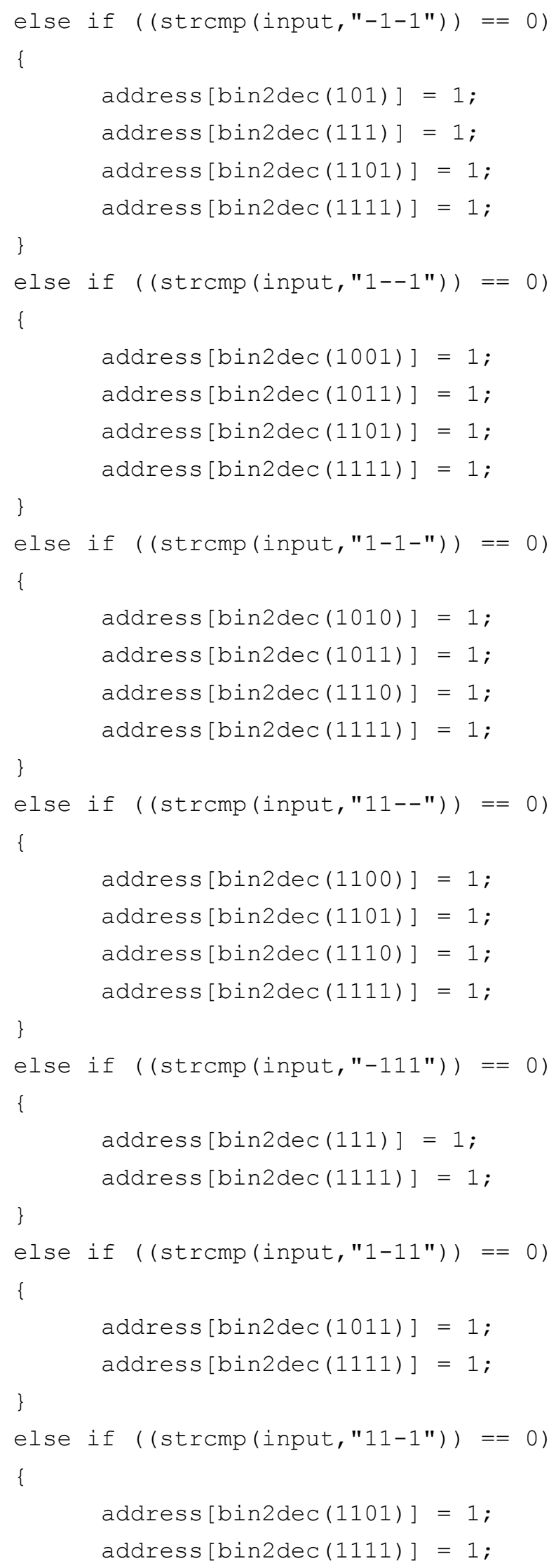




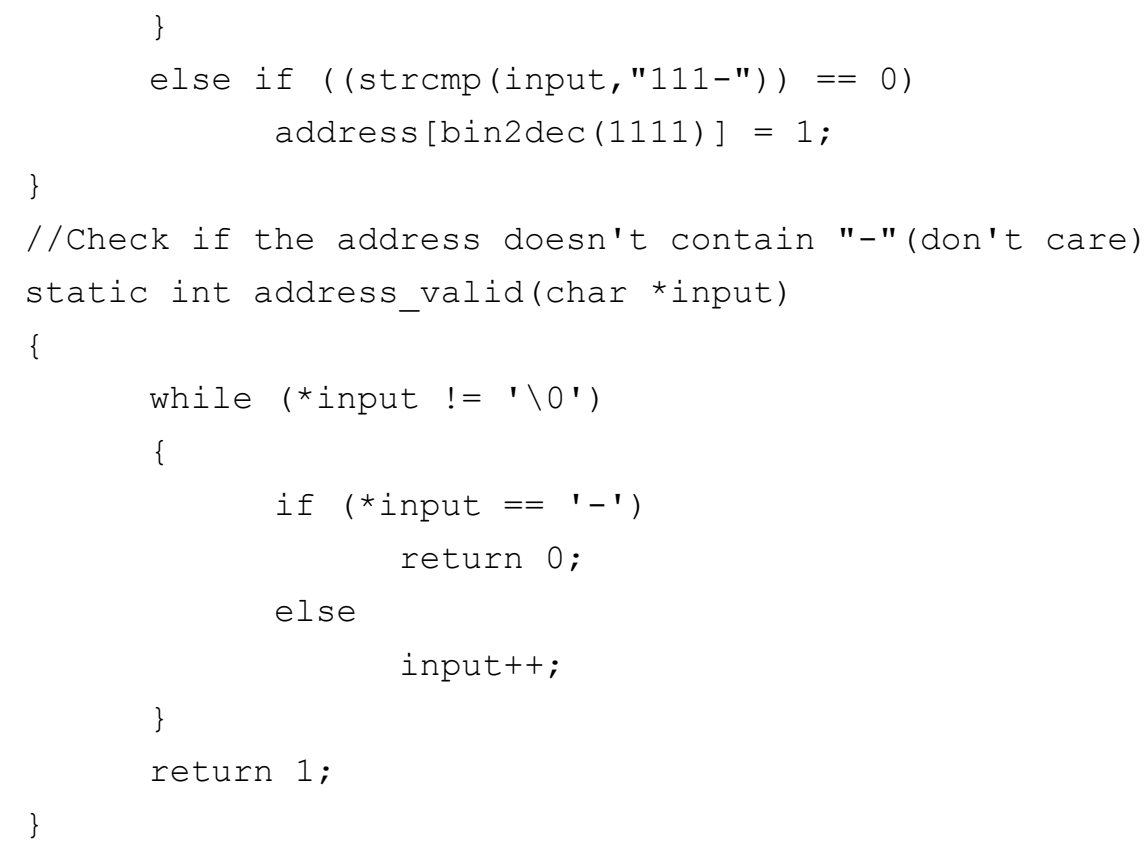




\section{util.c:}

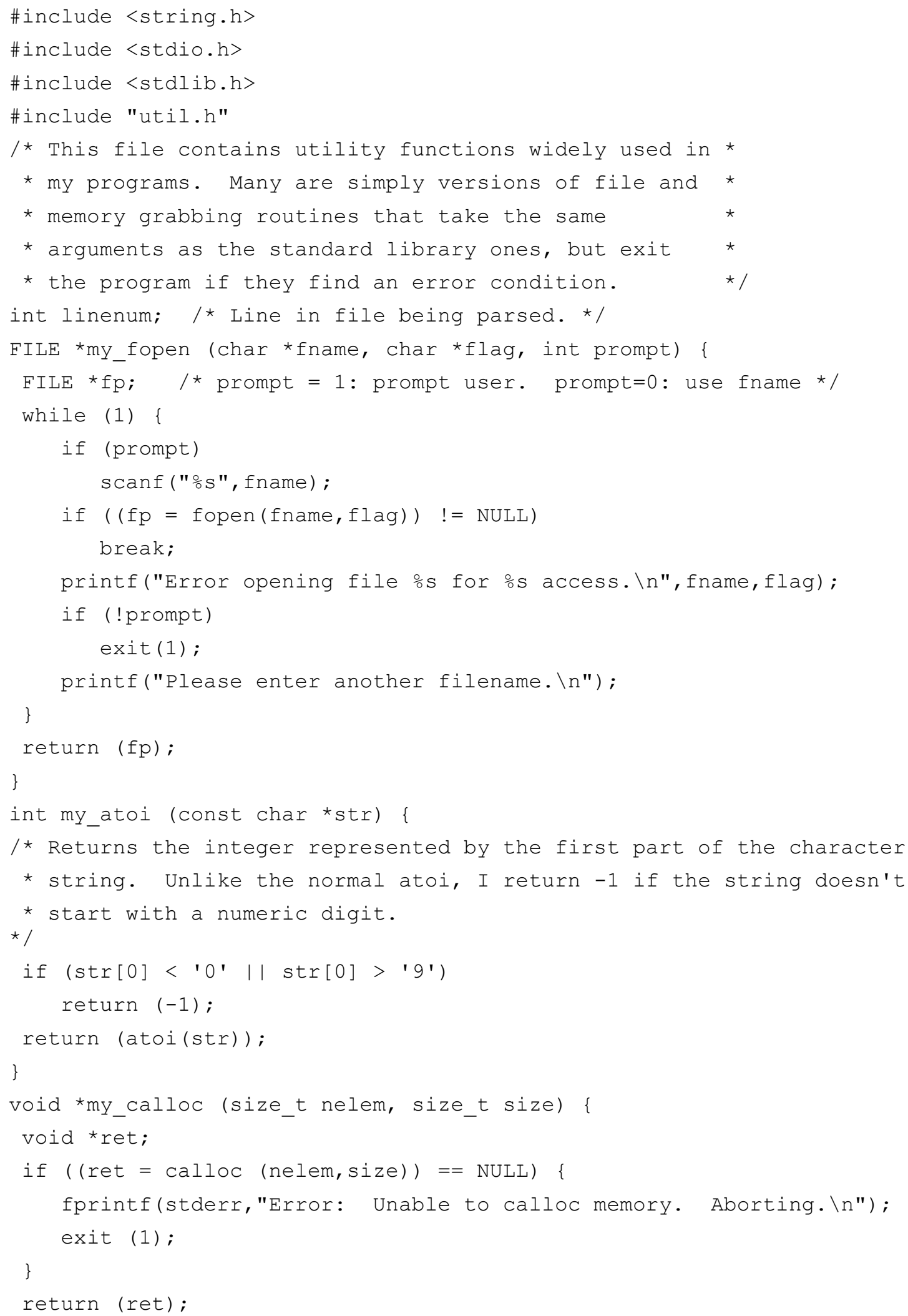




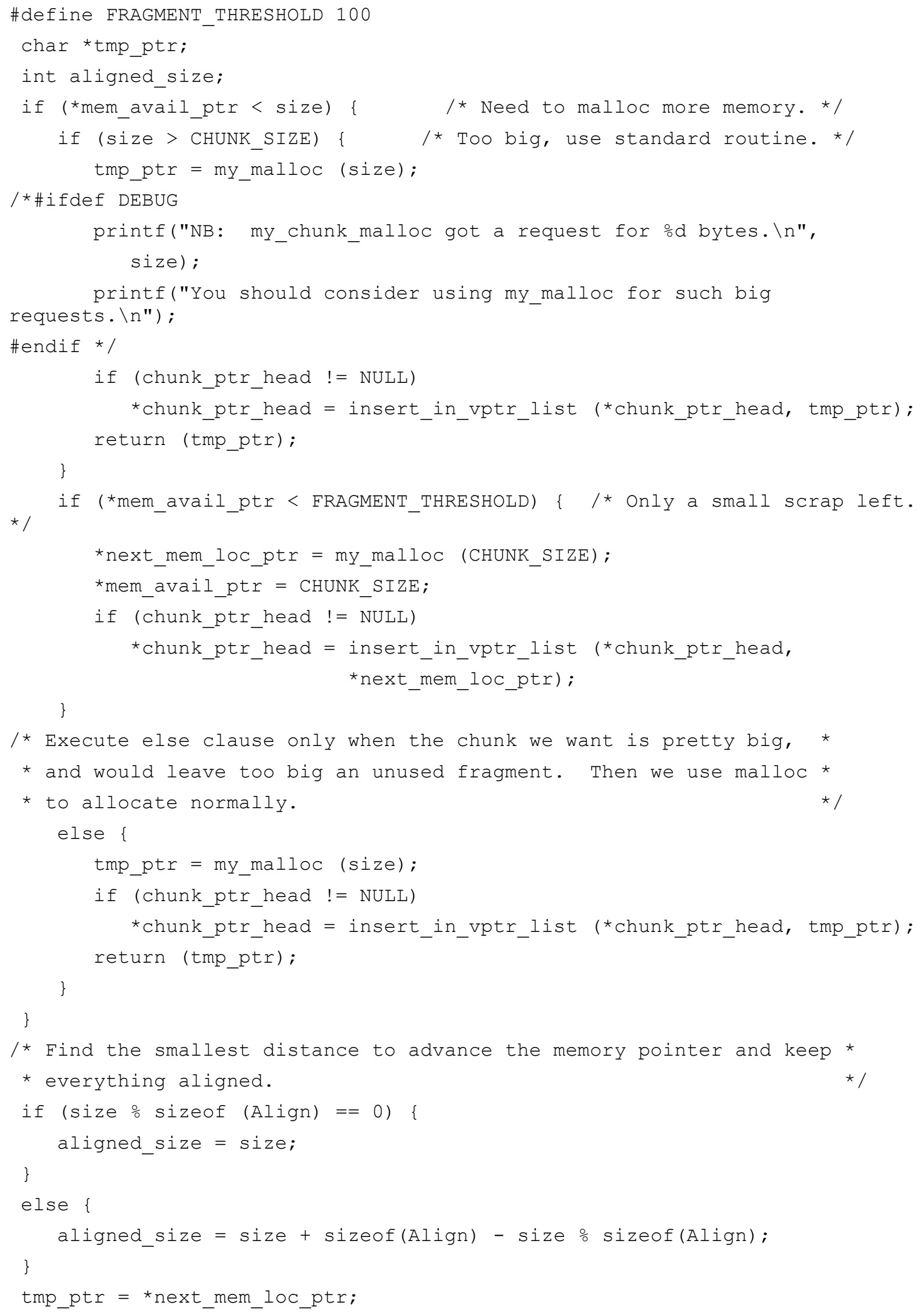




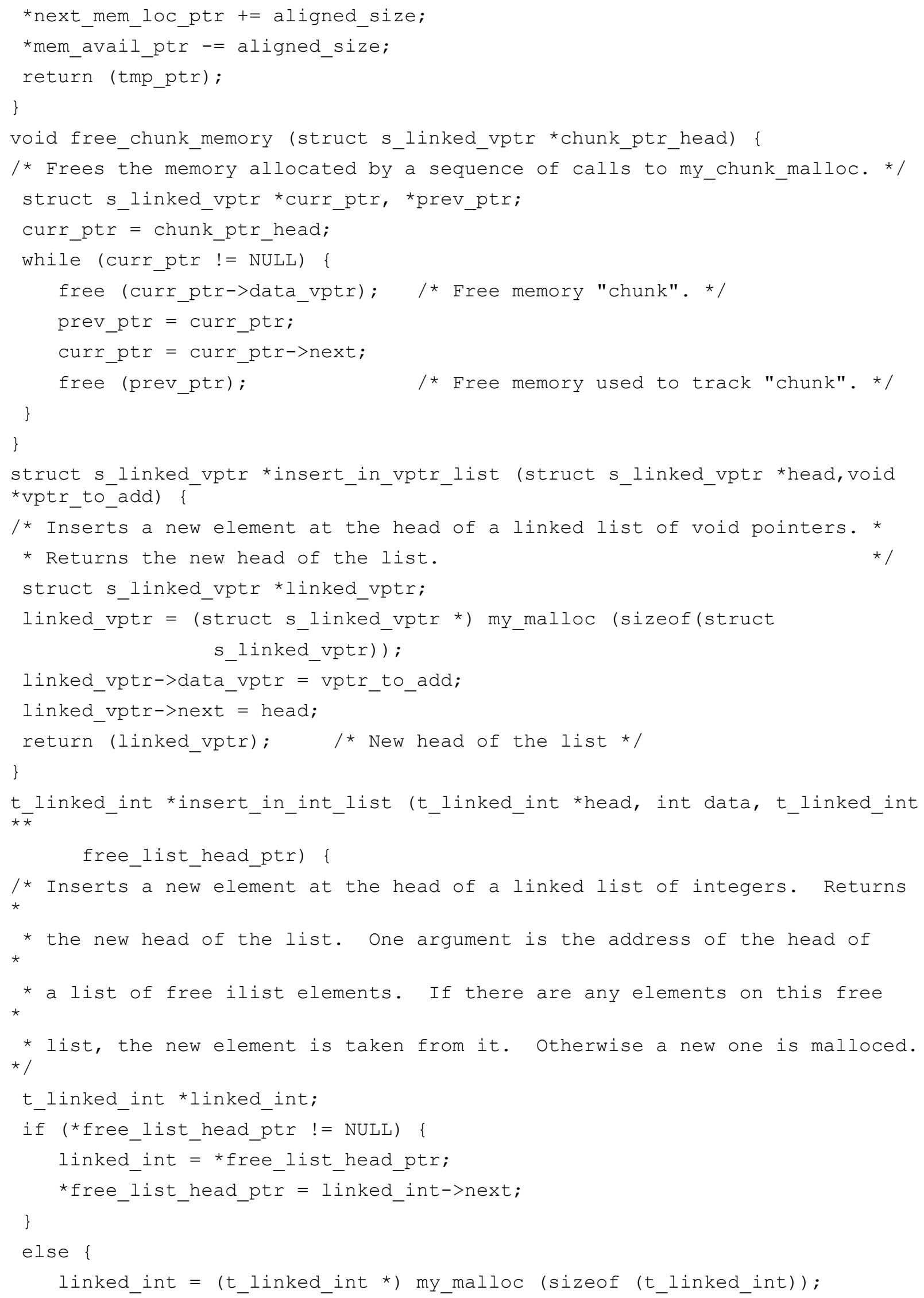




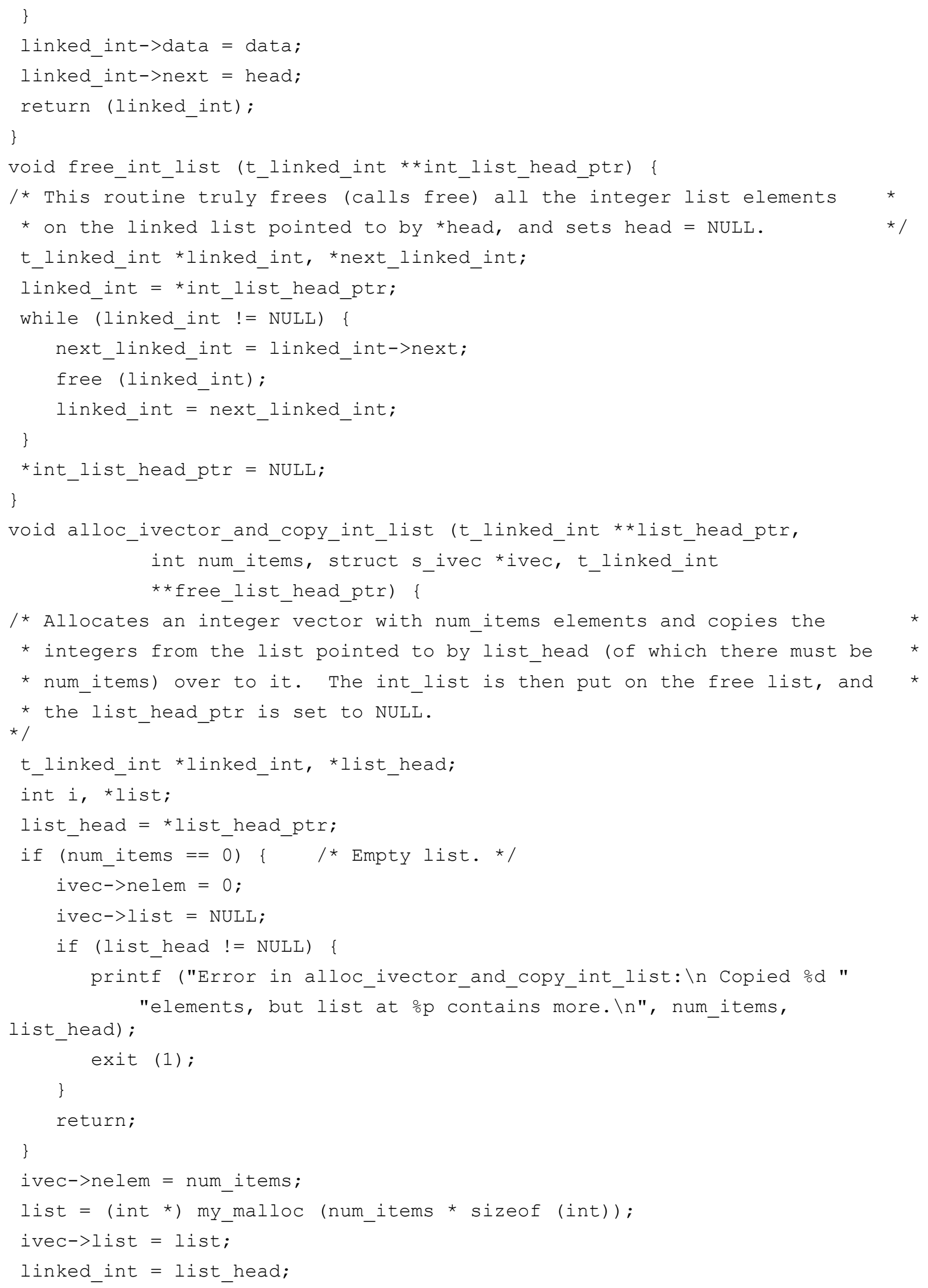




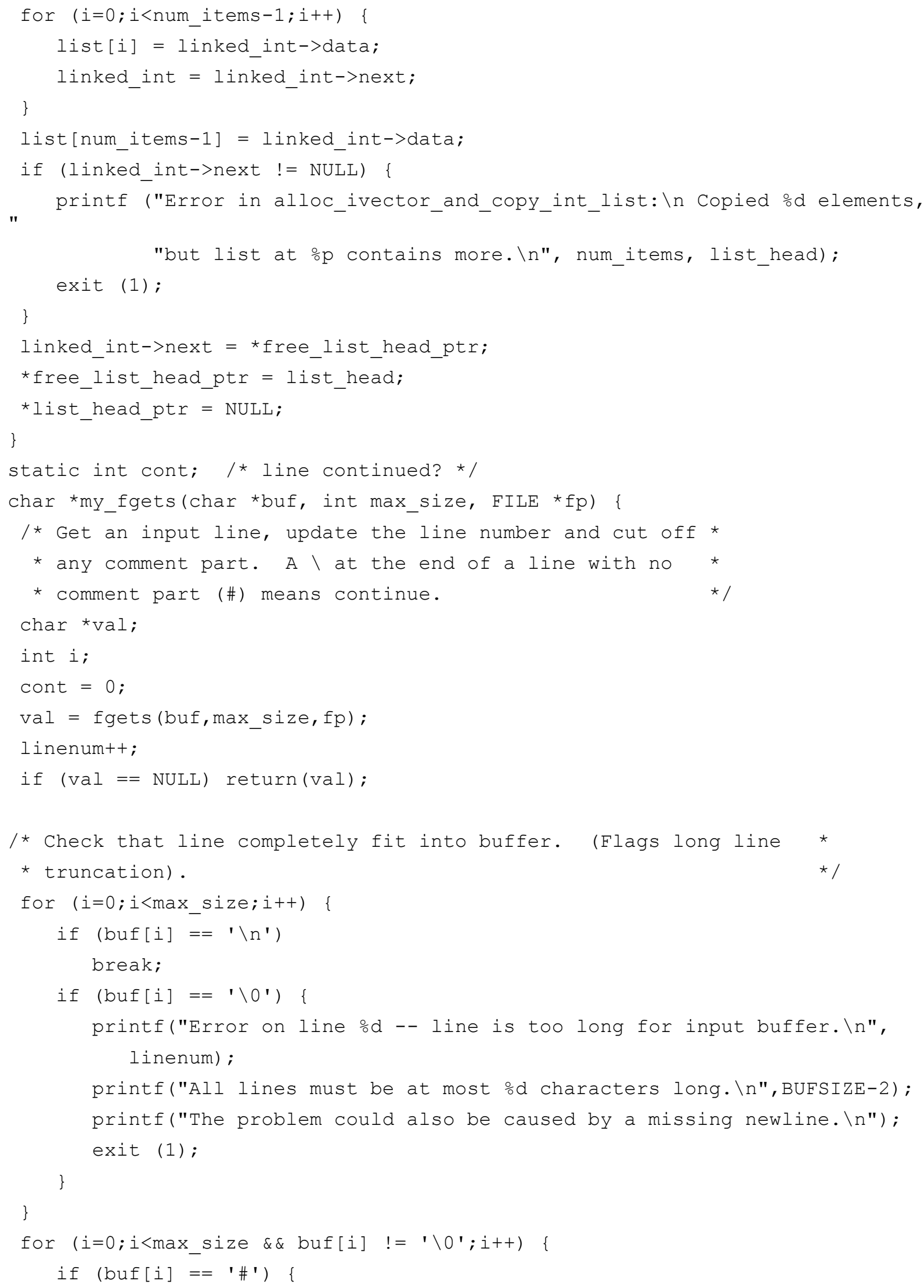




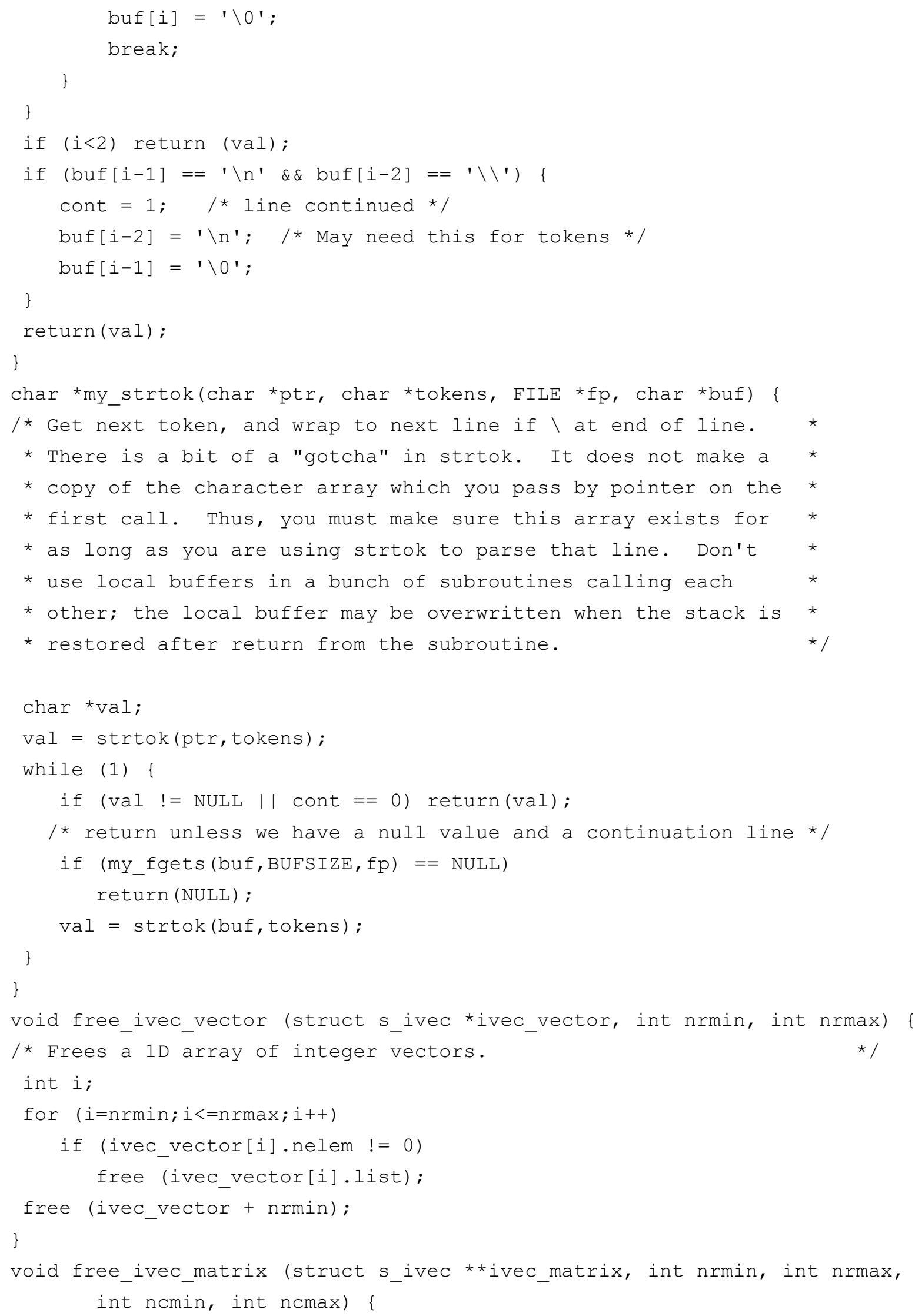




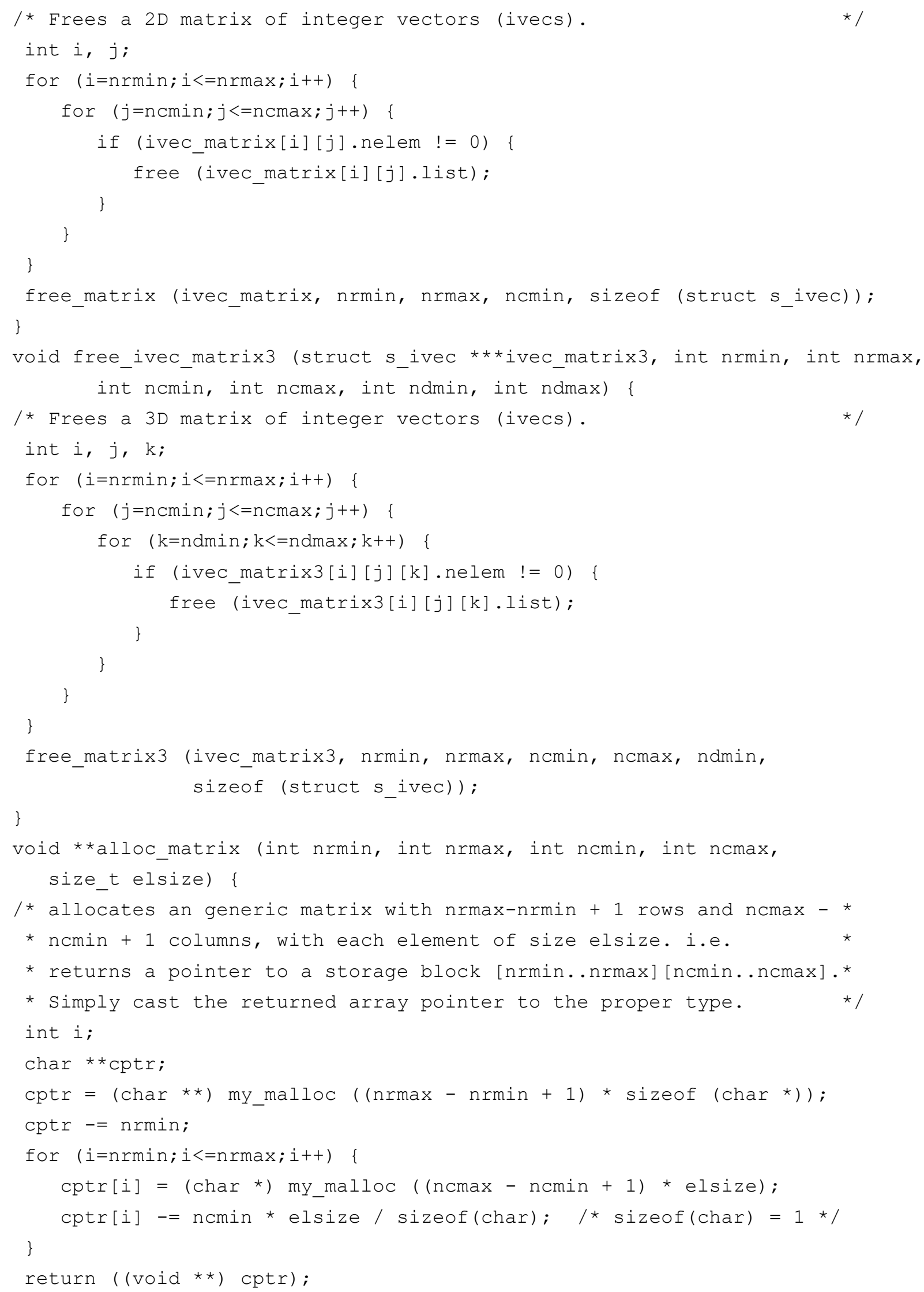


I* NB: need to make the pointer type void * instead of void ** to allow

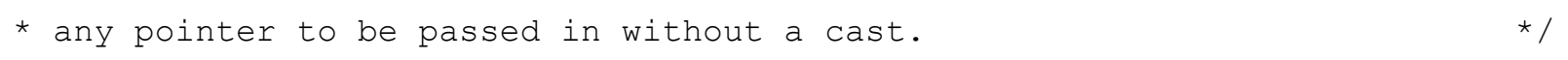




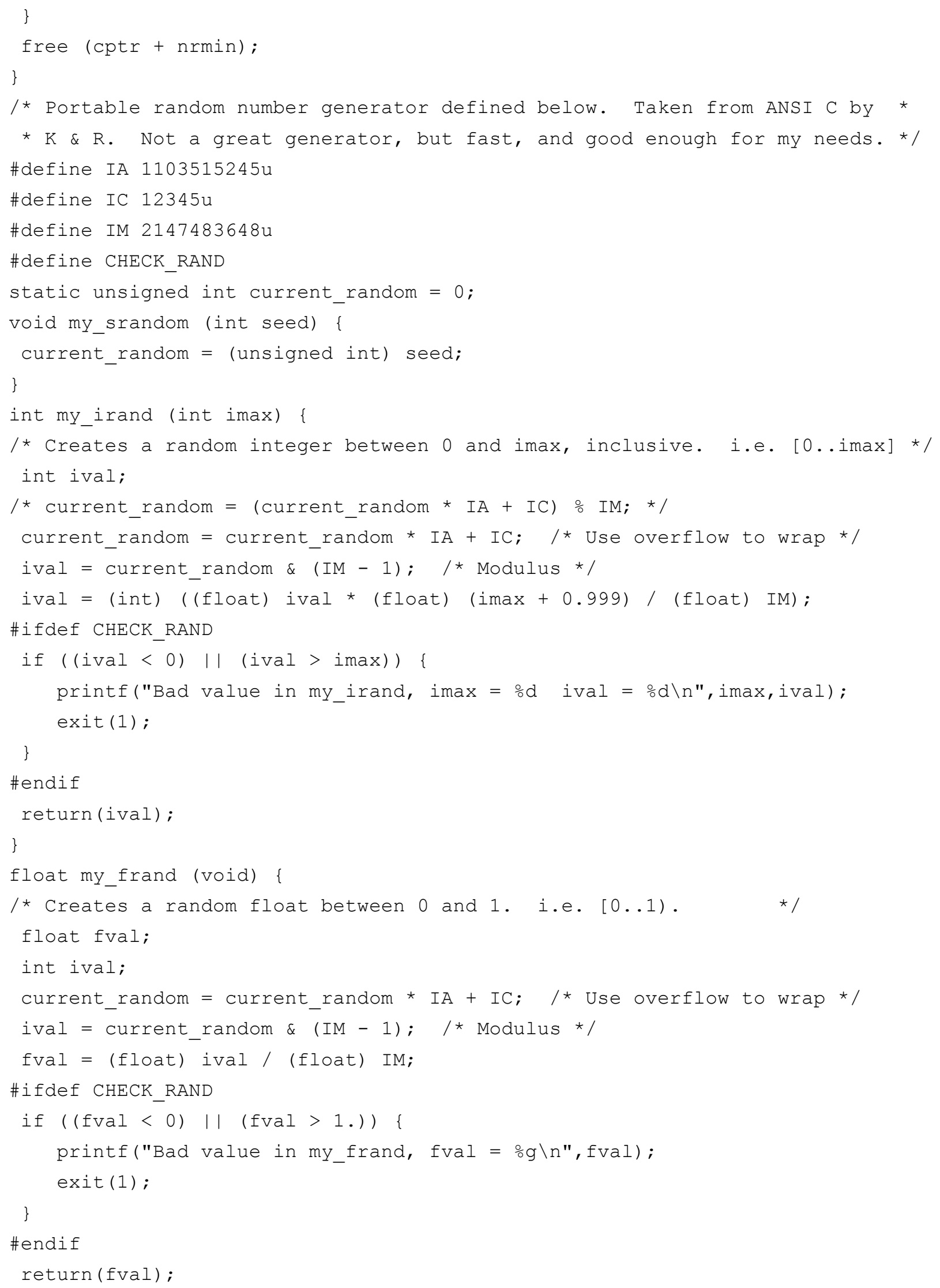




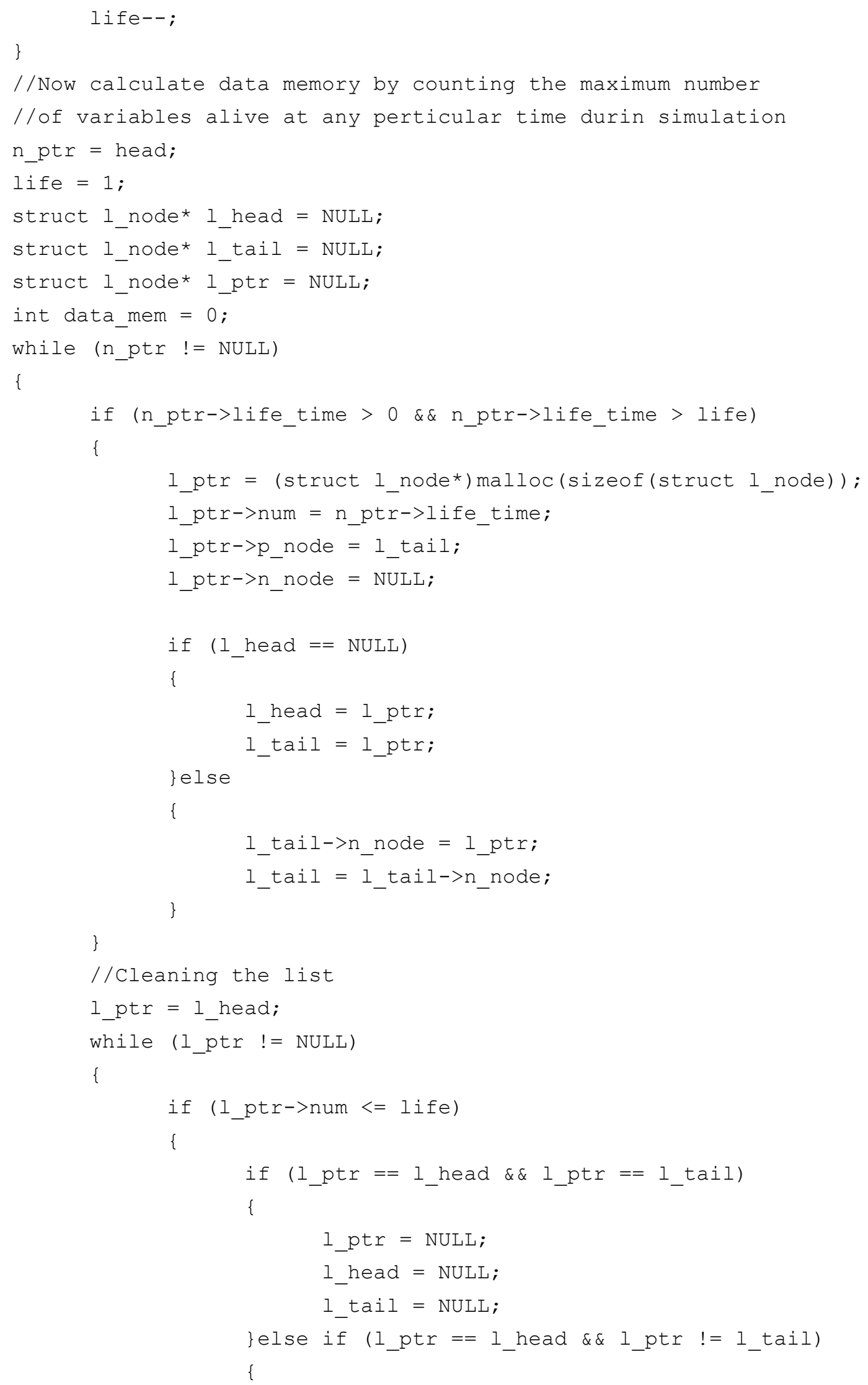




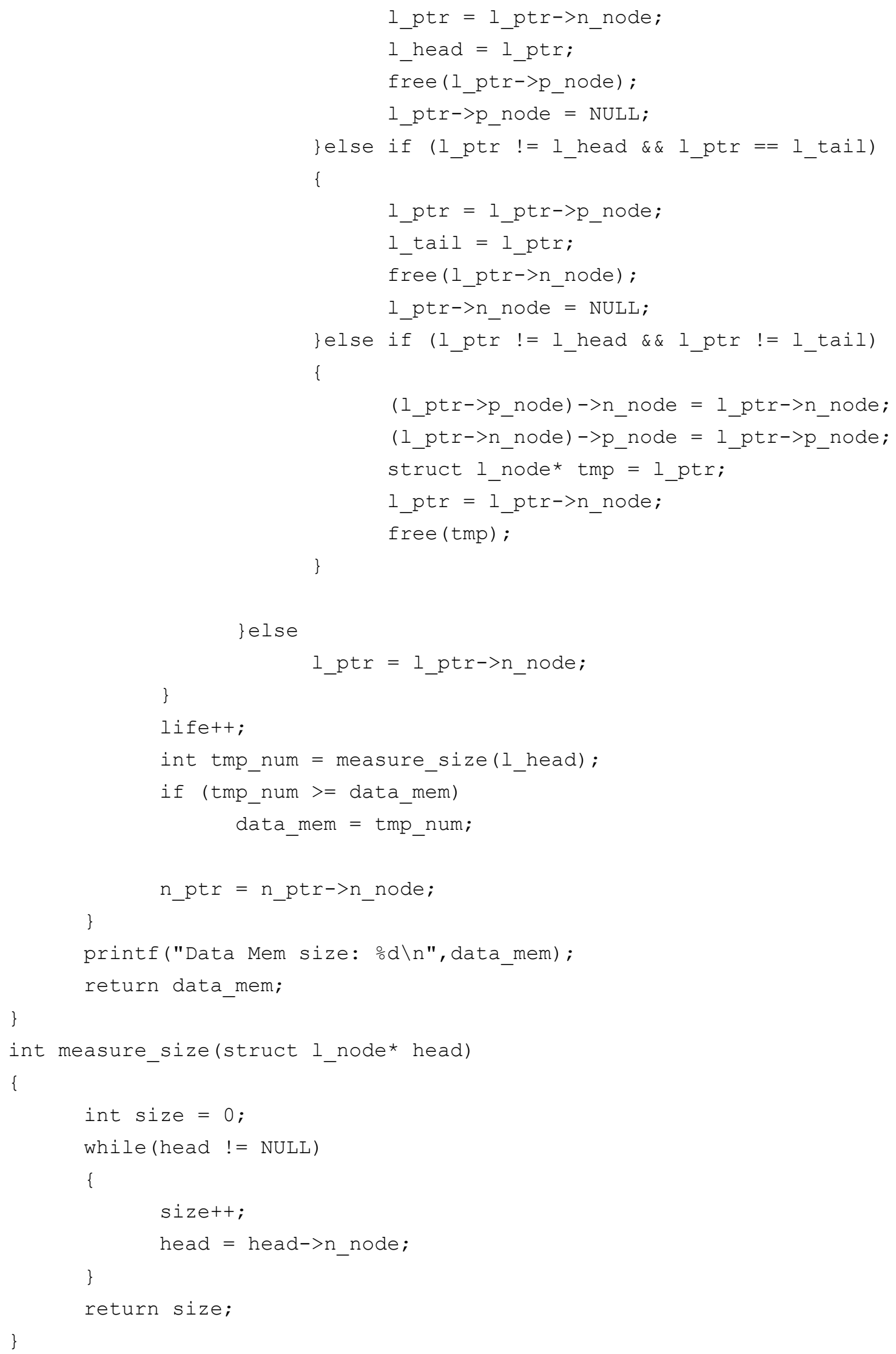




\section{graph.c:}

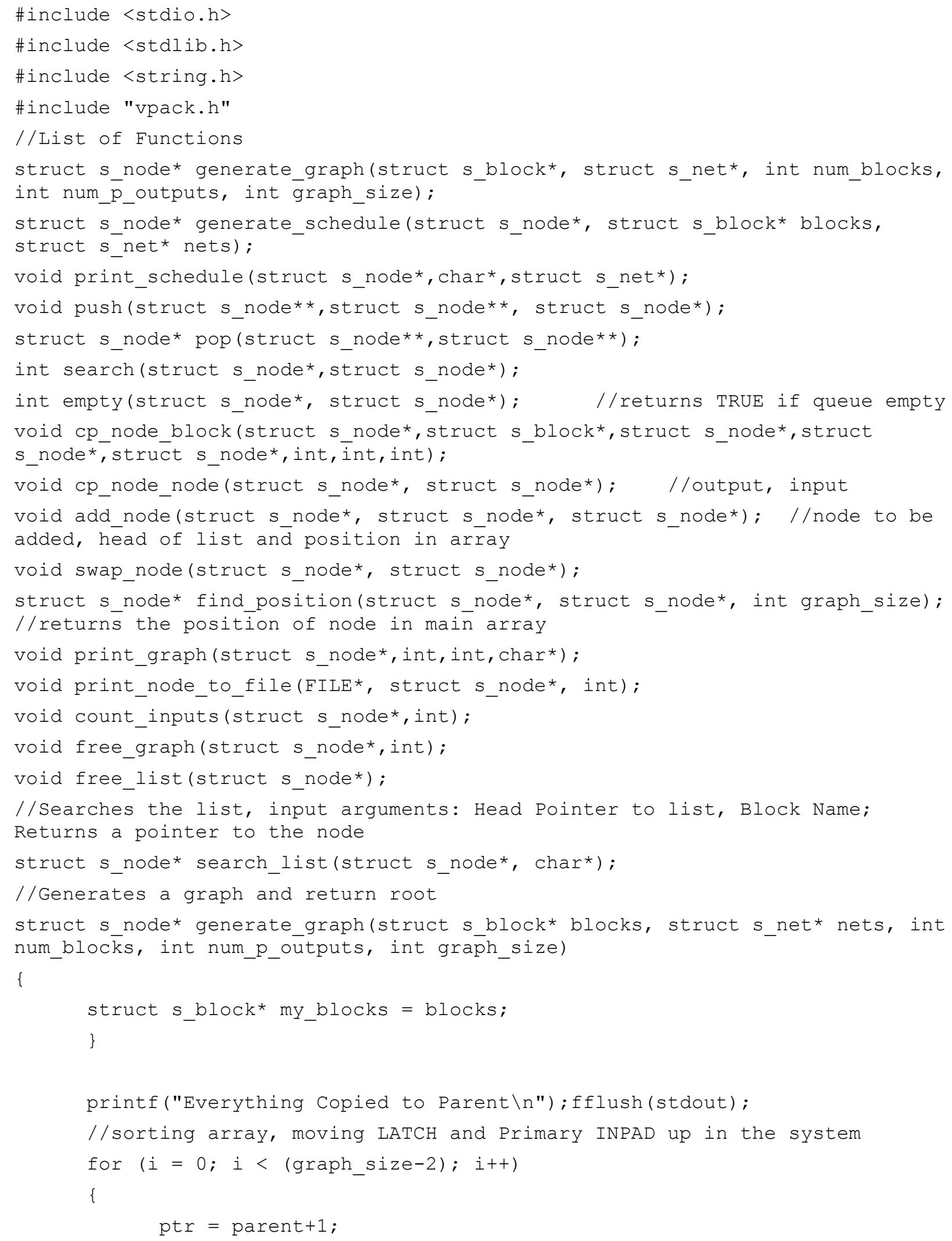




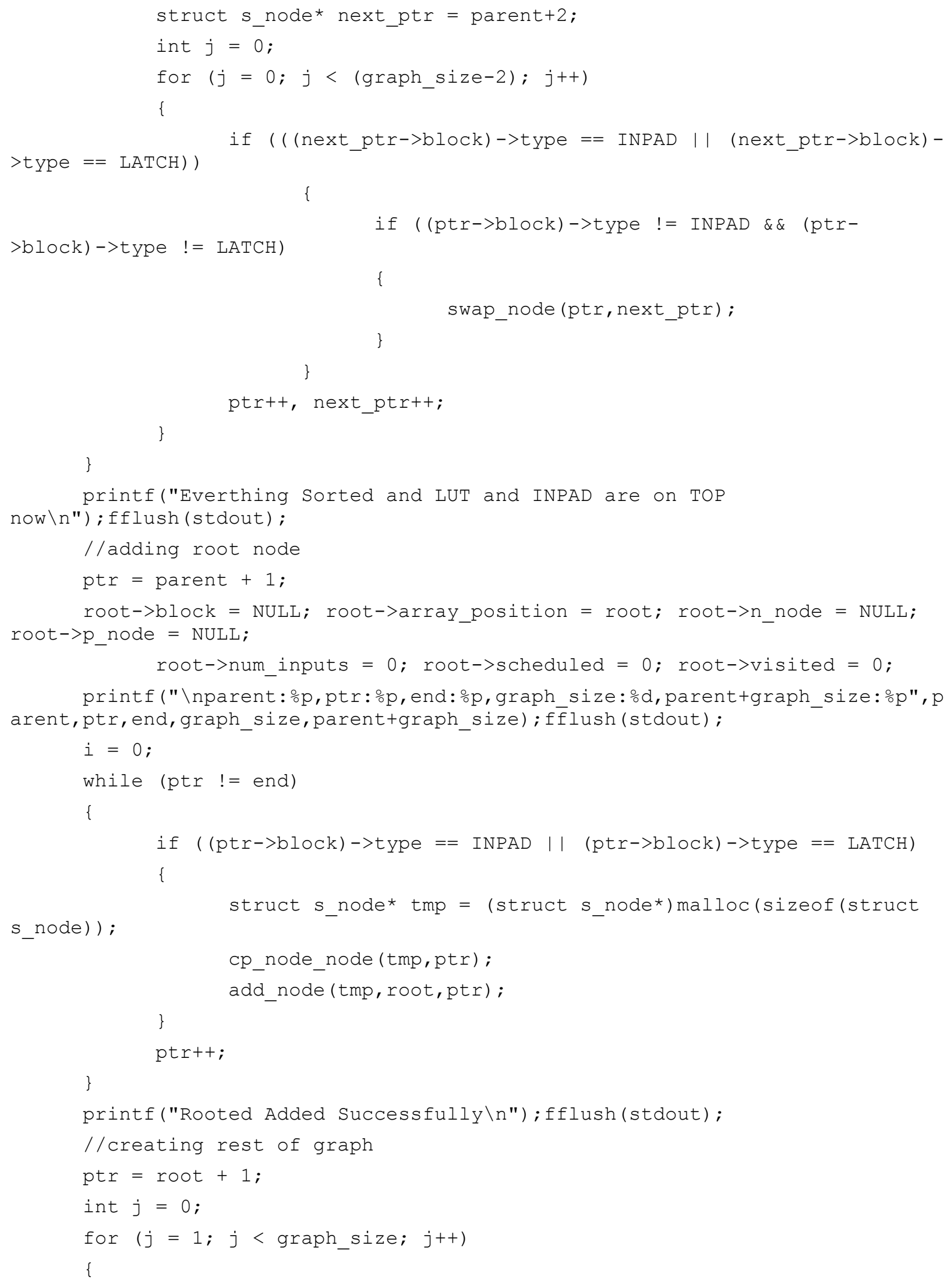




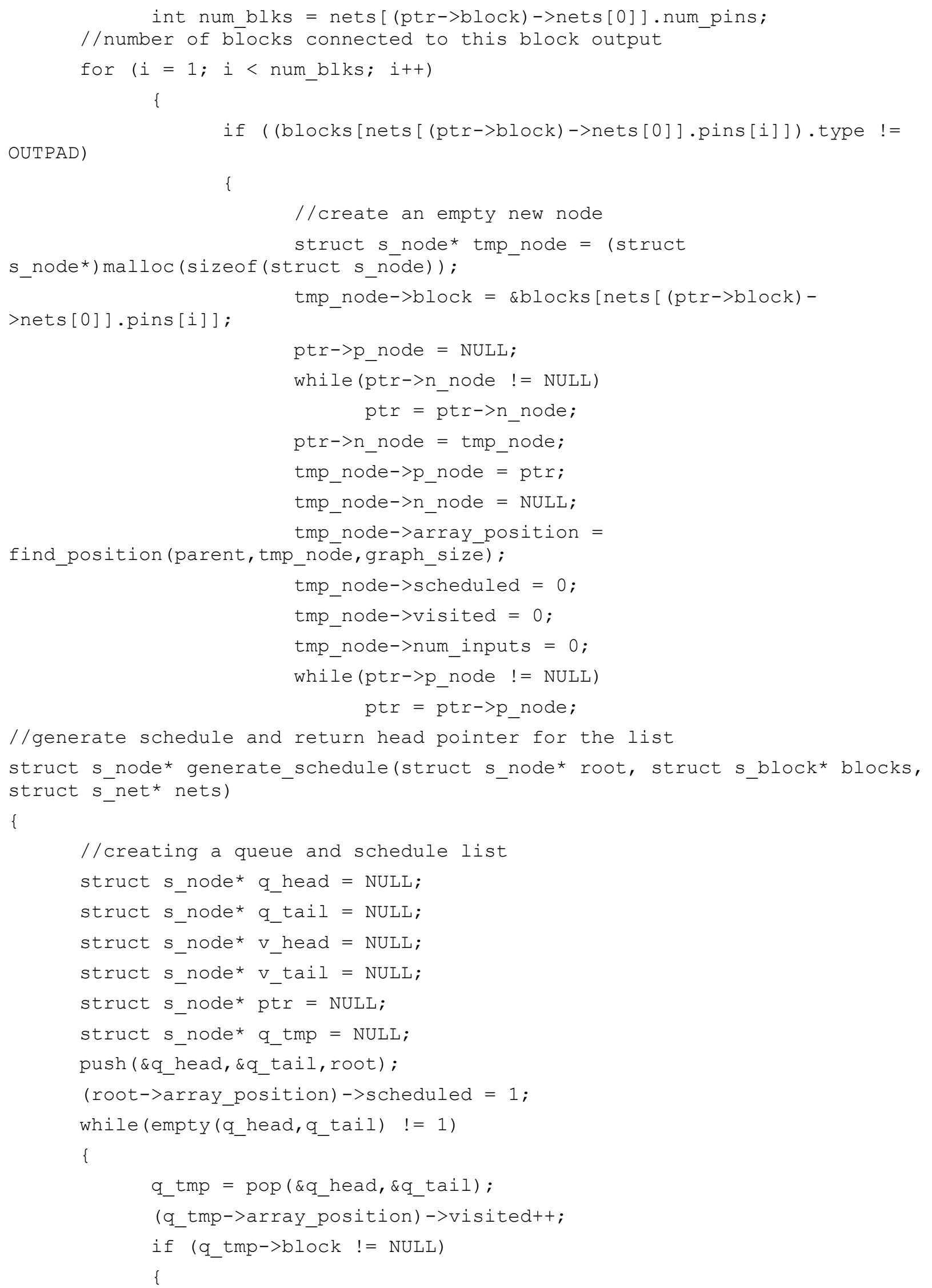


$==\mathrm{LATCH}$ )

if $\left(\left(\left(q_{-}\right.\right.\right.$tmp->block $)->$type $==$INPAD $)$|| (q_tmp->block)->type

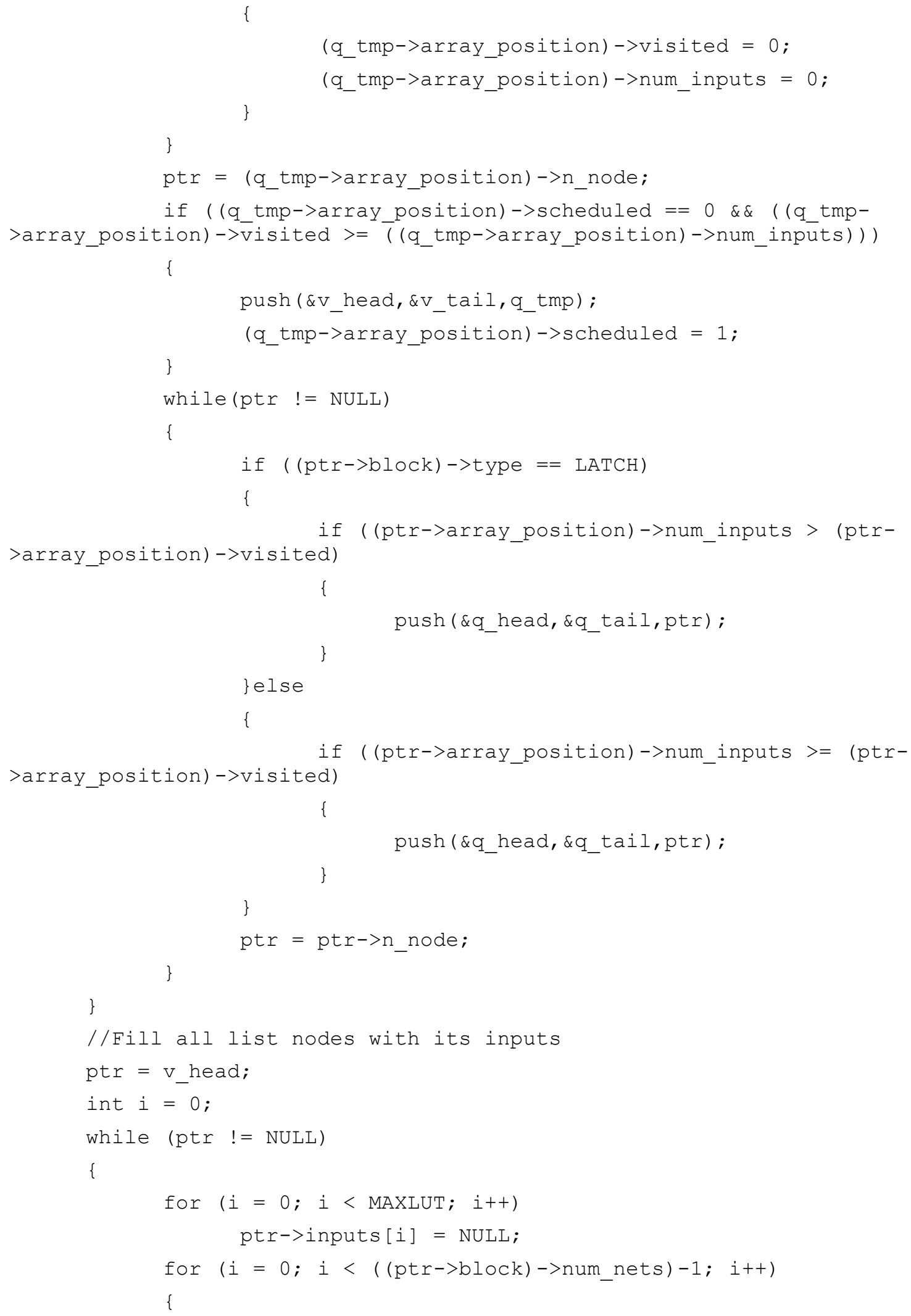




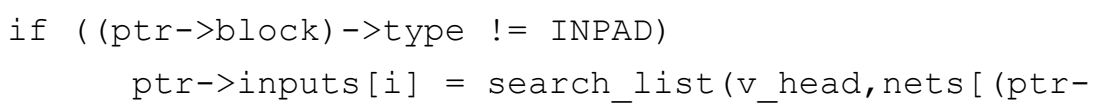




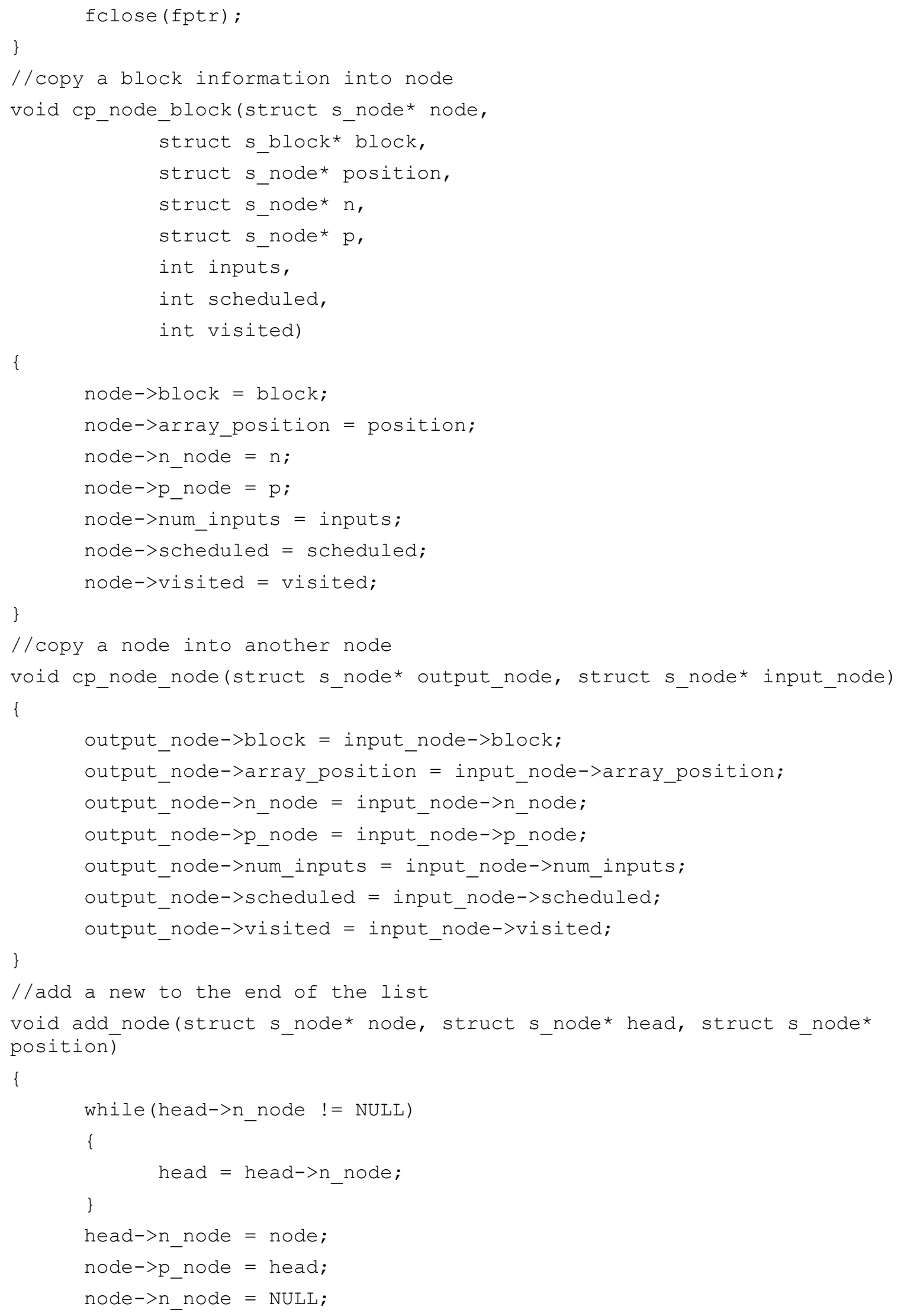




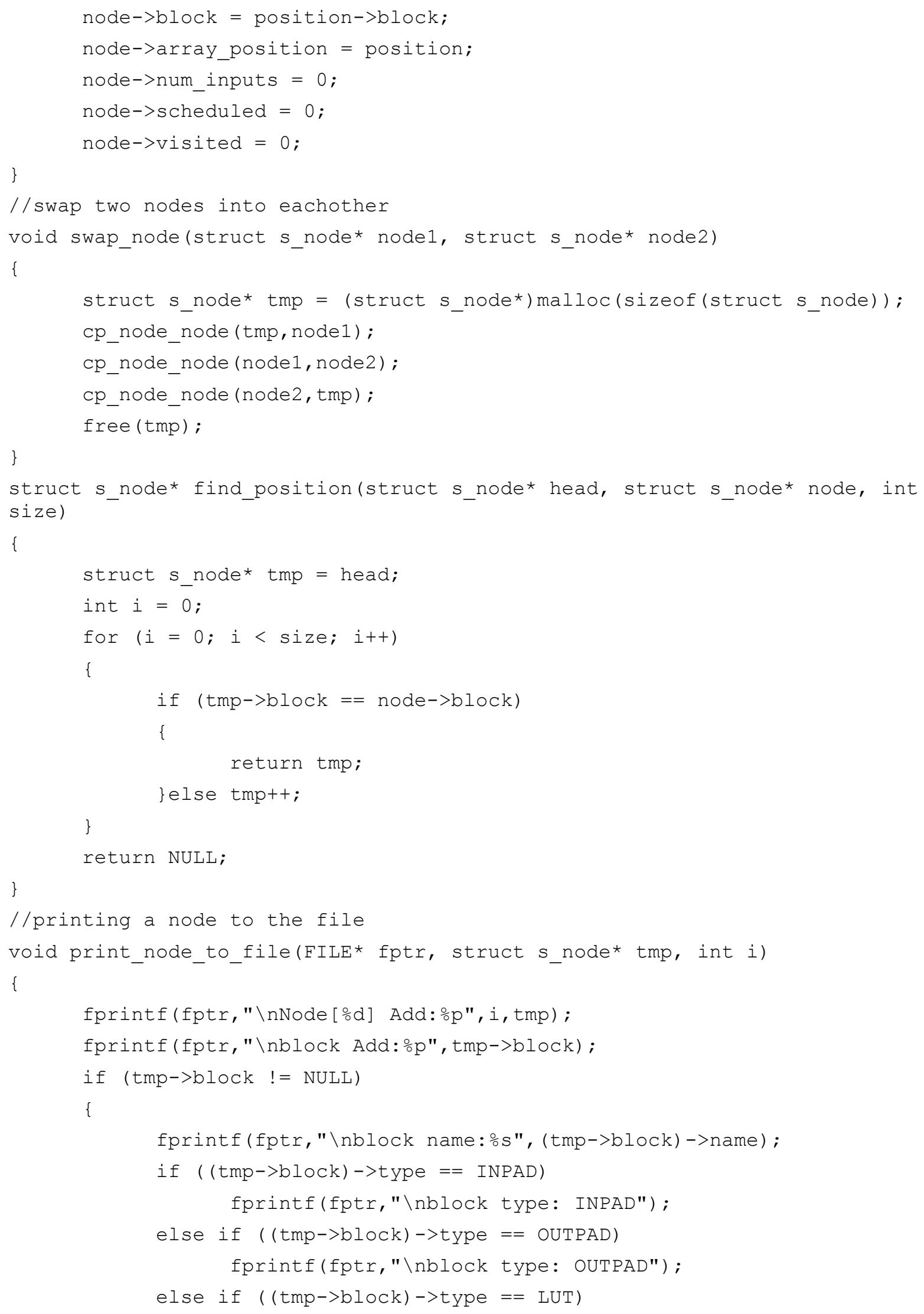




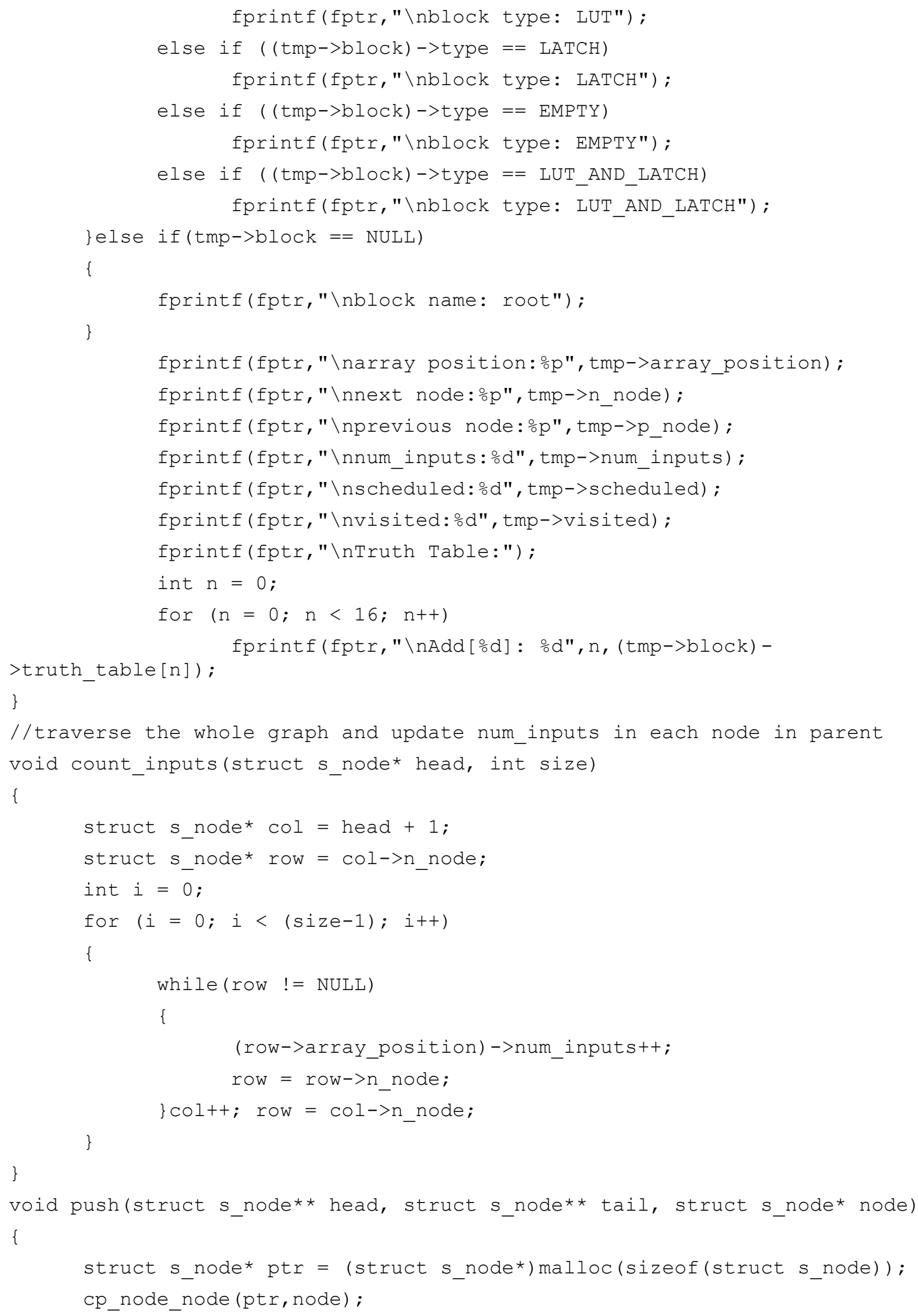




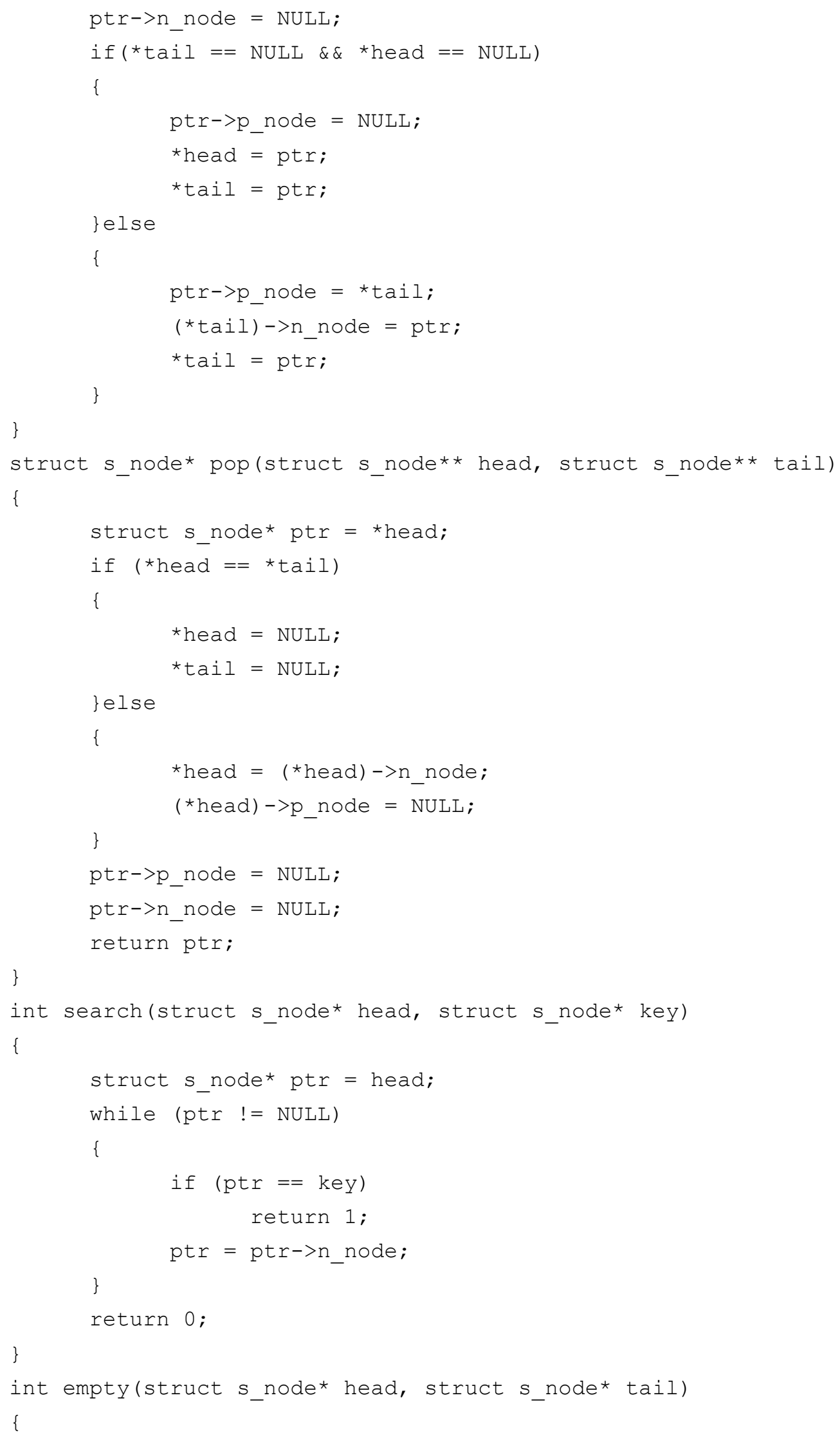




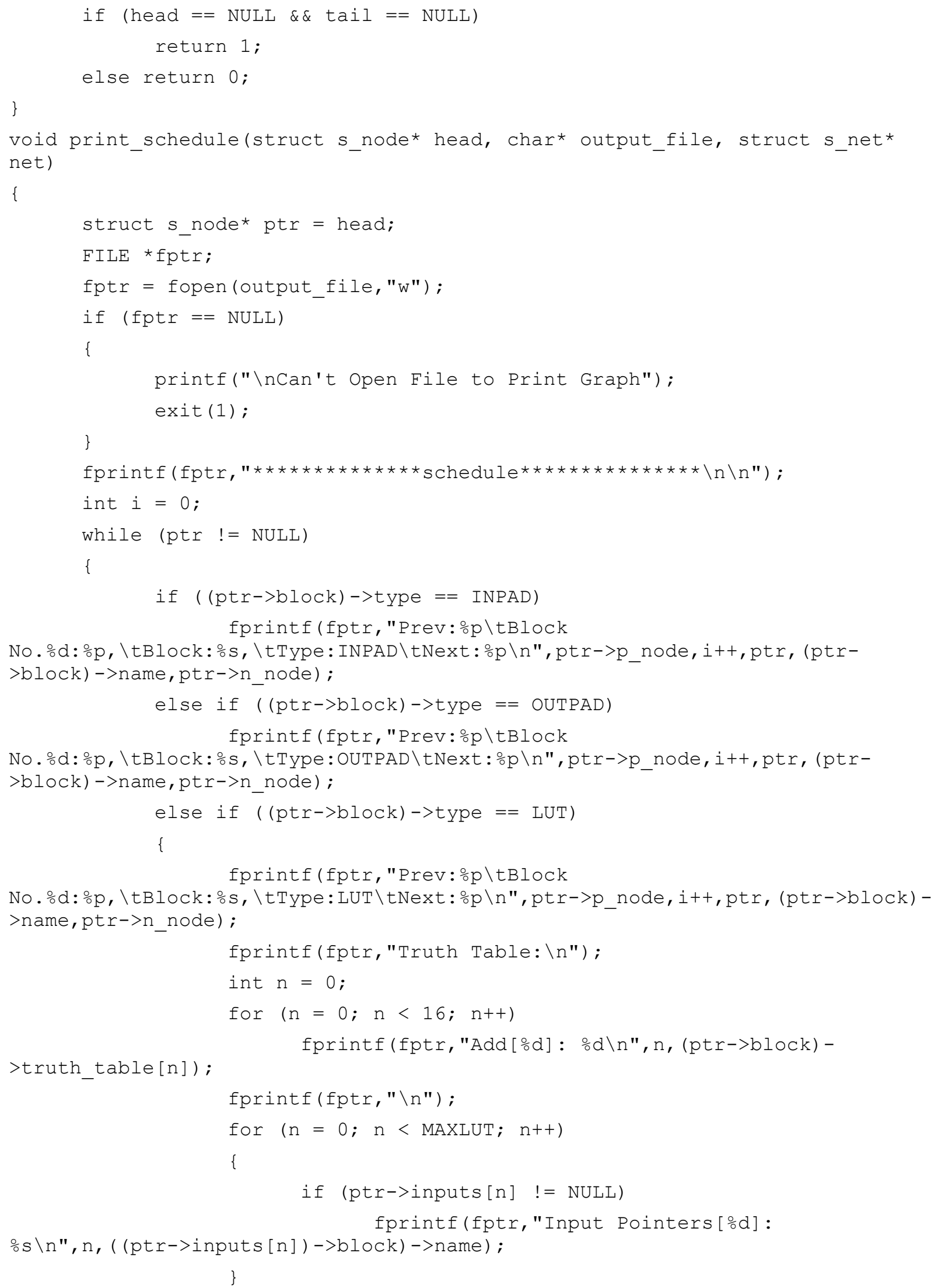




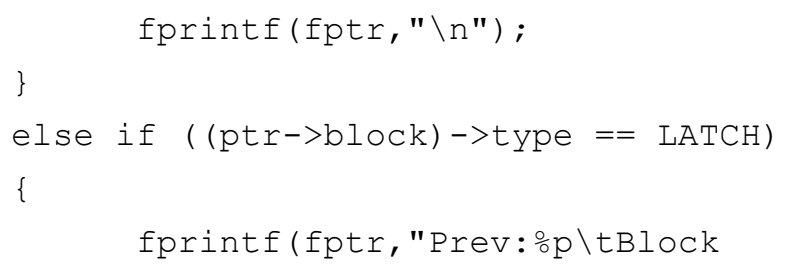




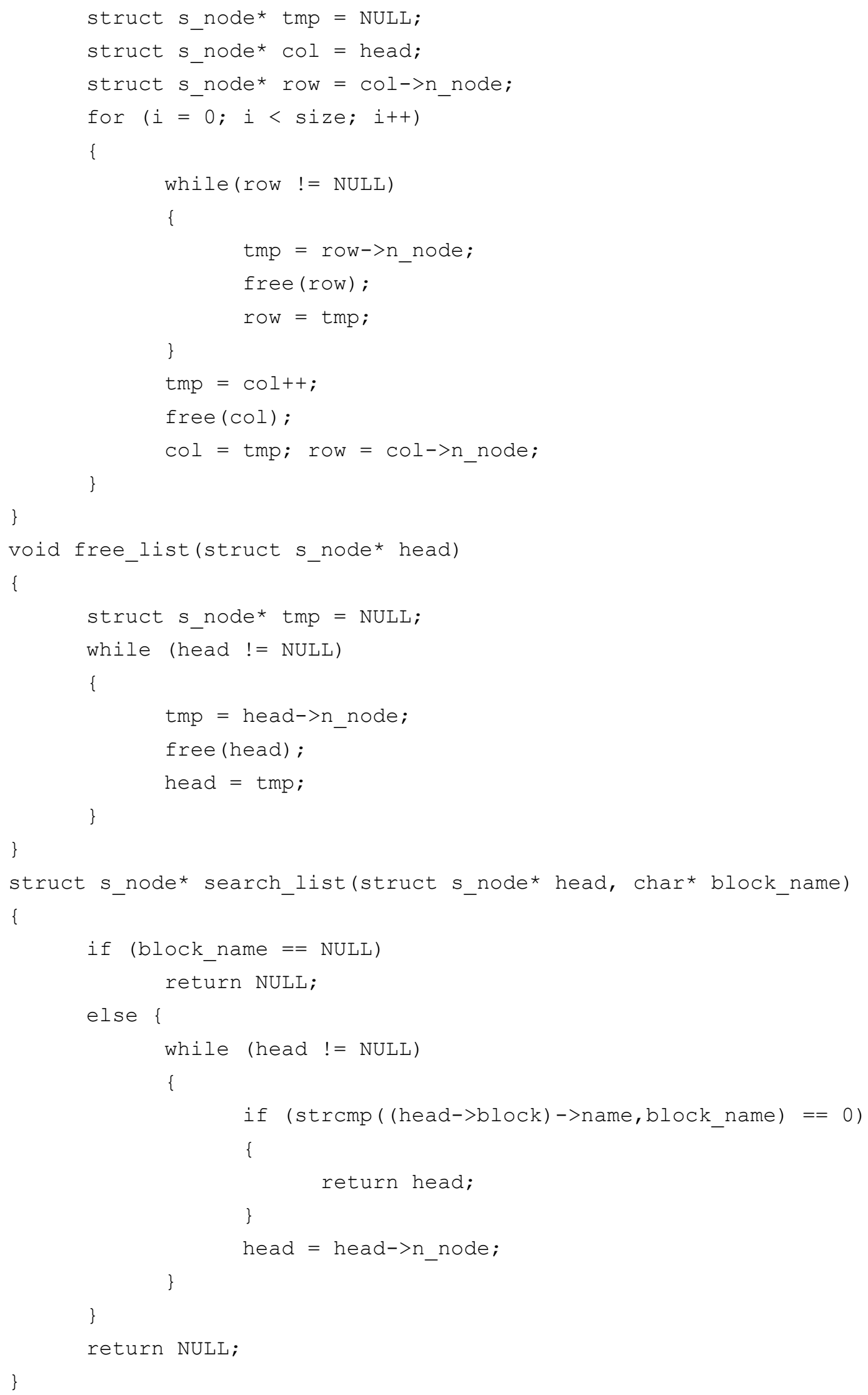




\section{simulation.c:}

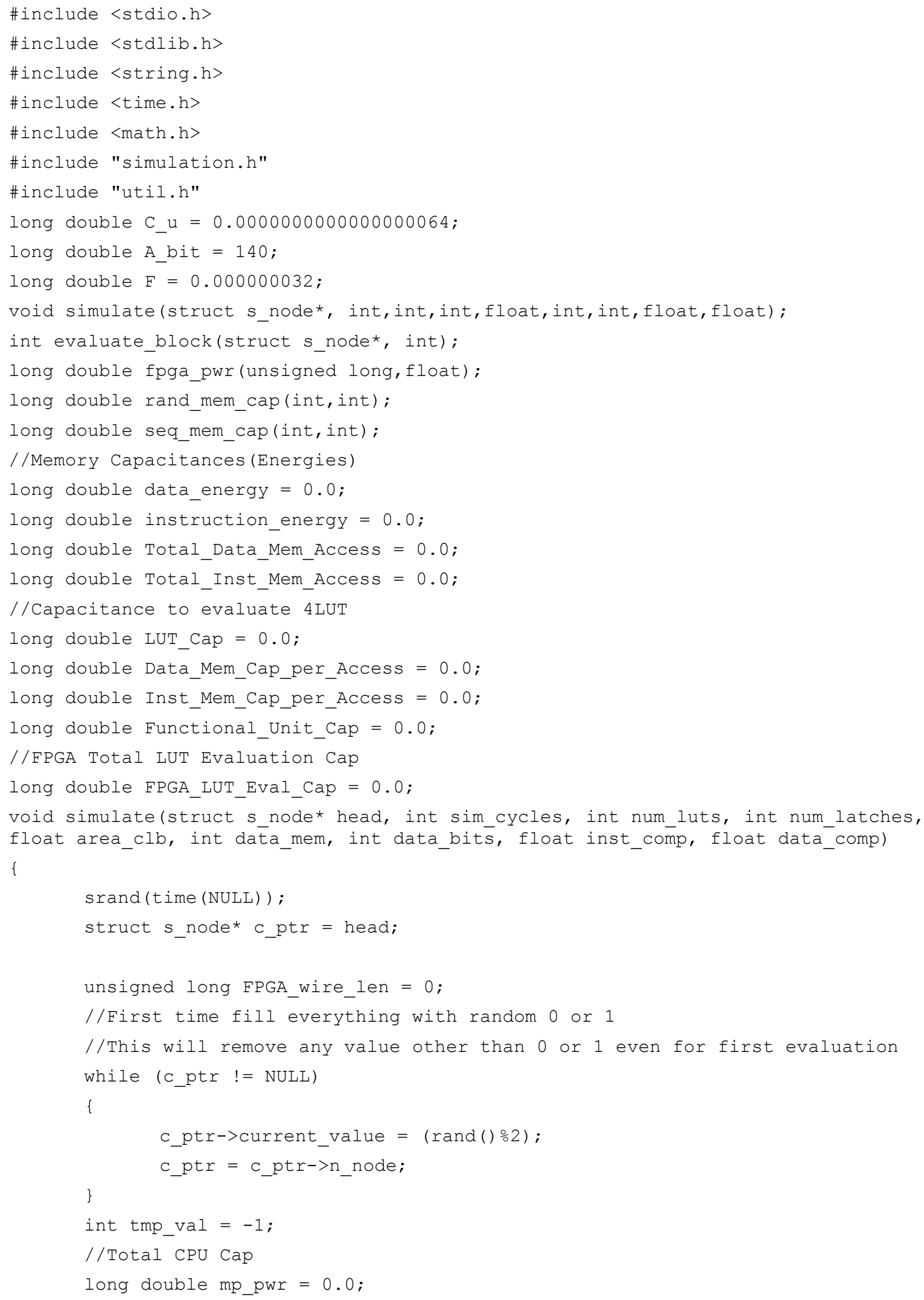




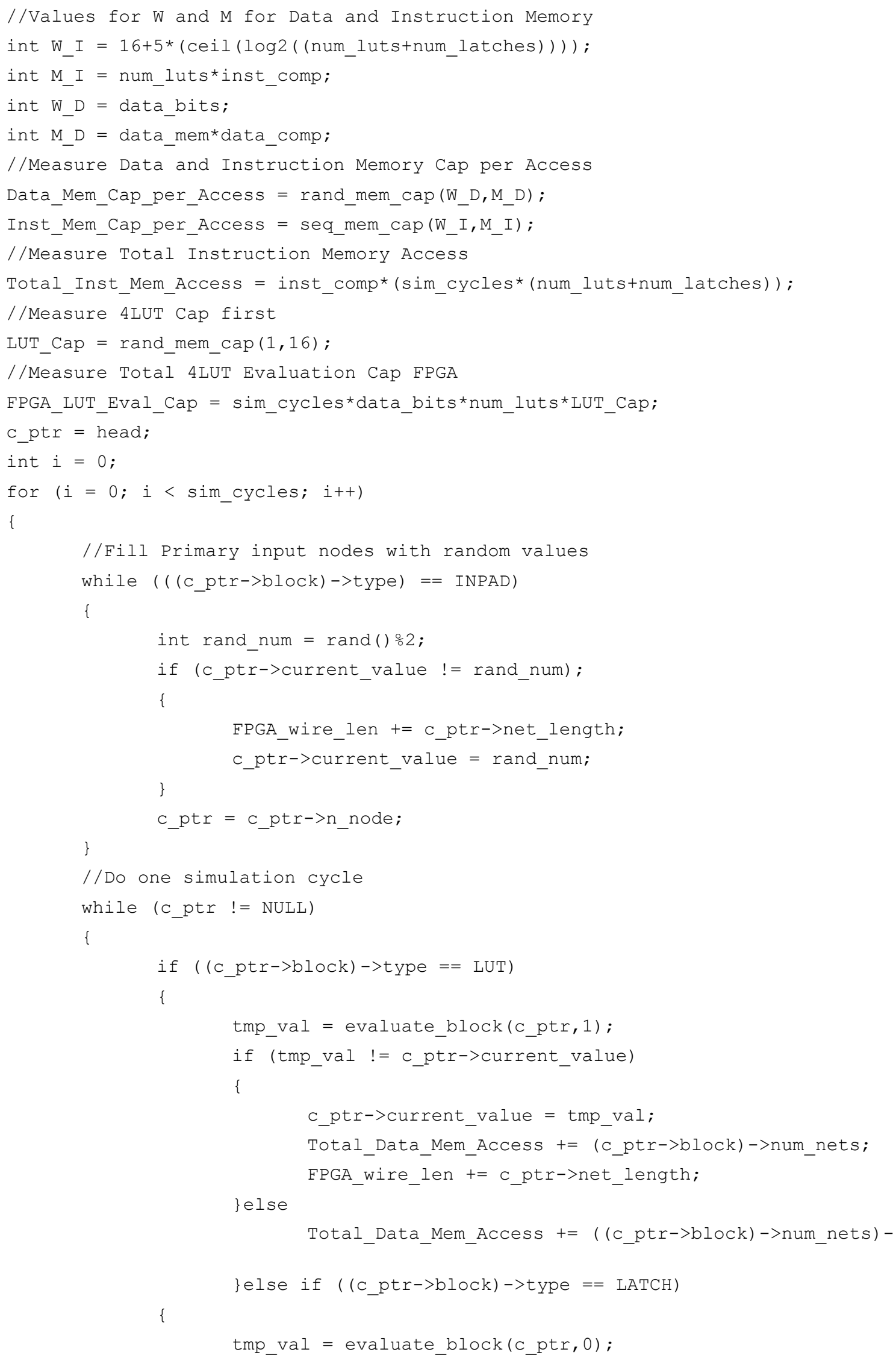




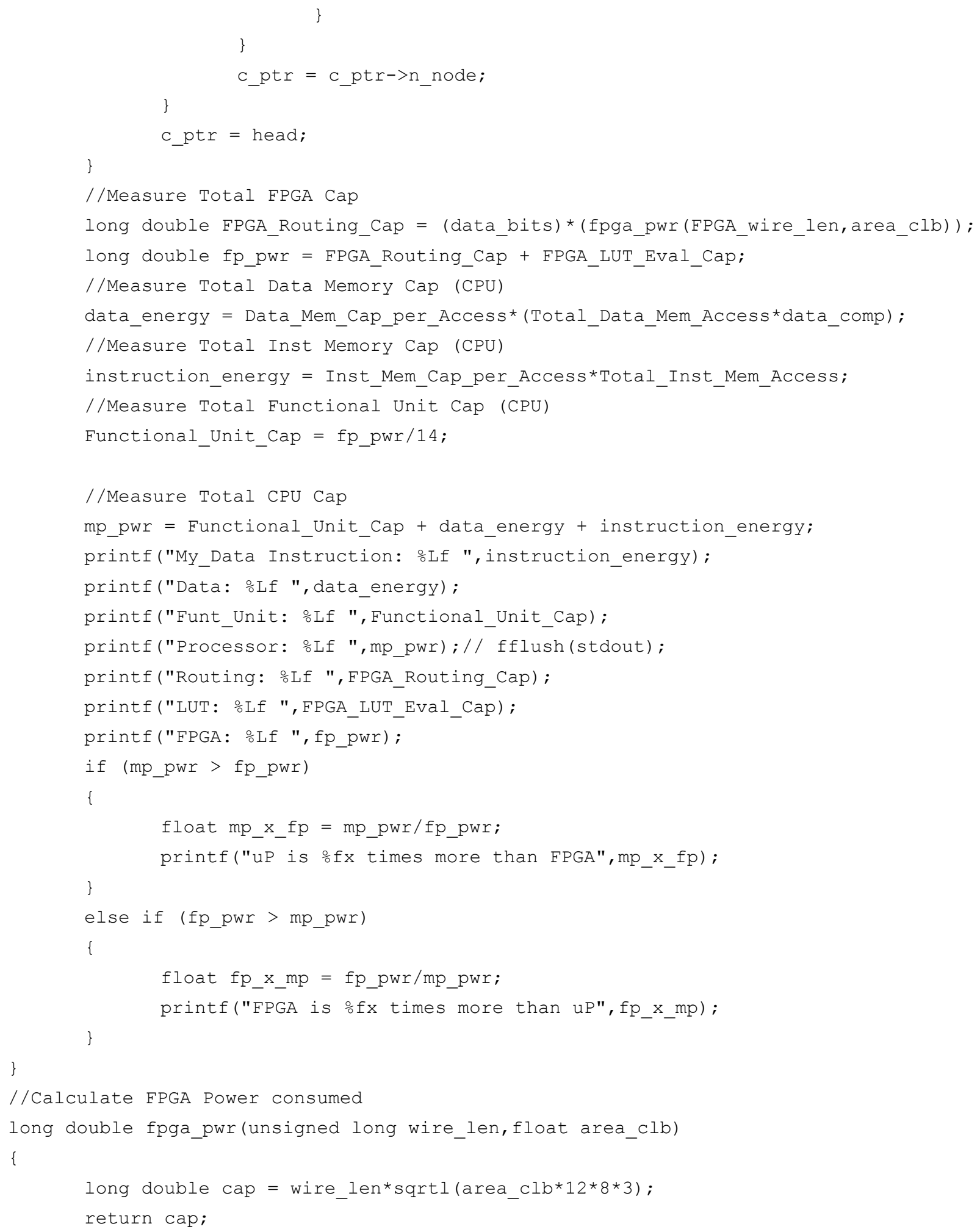


\}

//Calcualte Random Access Memory Capacitance

long double rand_mem_cap (int $W$, int $M$ )

\{

long double tmp_log $=(\log l(\mathrm{M})) /(\log 1(2))$;

long double cap $=($ tmp_log $+(2 *(2 * W+2))) *\left(\operatorname{sqrtl}\left(W^{*} M\right)\right)$;

return cap;

\}

//Calculate Seq Access Memory Capacitance

long double seq_mem_cap (int $W$, int $M$ )

\{

long double cap $=((2 *(2 * W+1)) * \operatorname{sqrt} l(W * M))$;

return cap;

\}

//Evaluate current block value based on its input values

int evaluate_block(struct s_node* c_ptr, int type)

\{

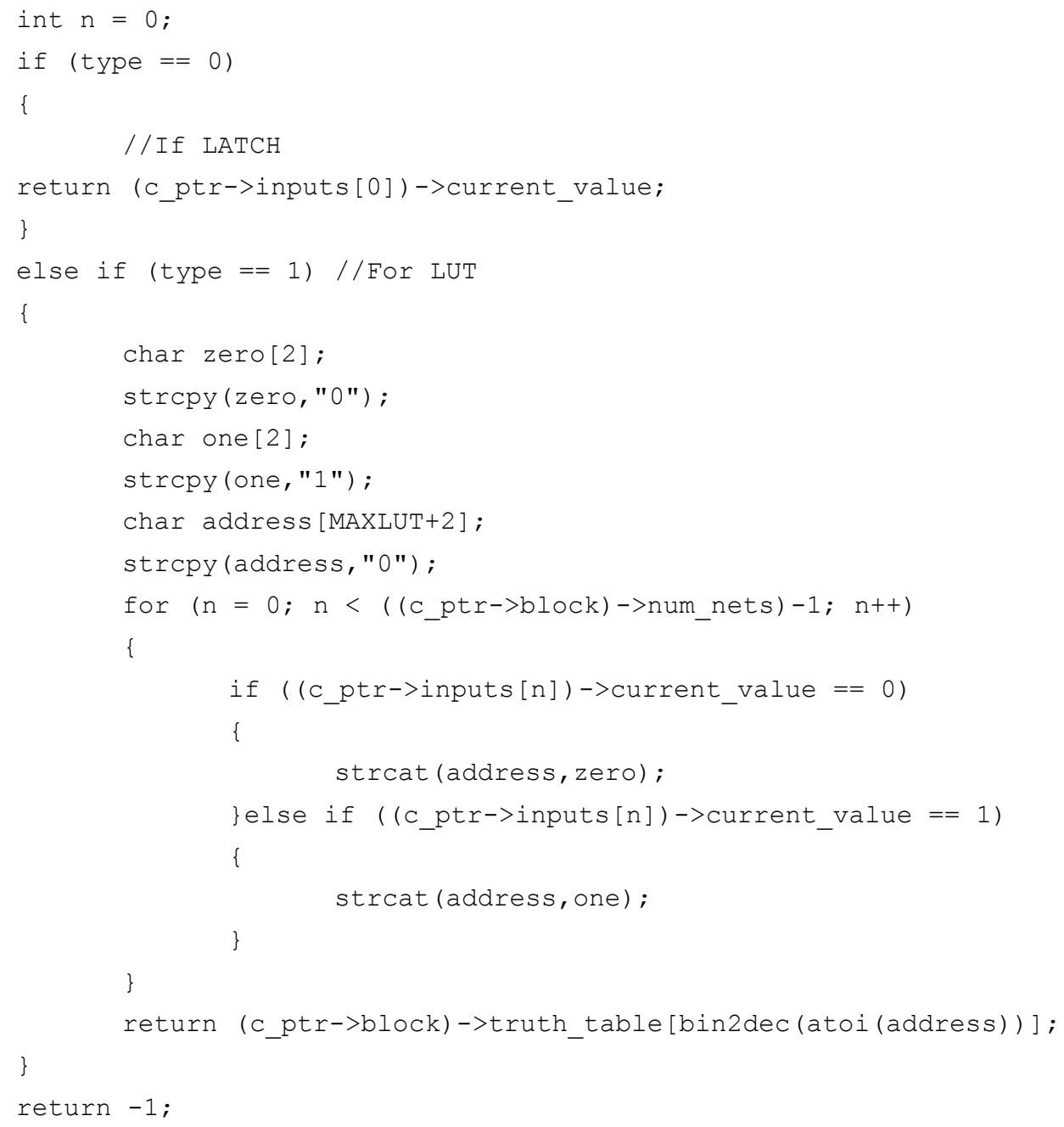




\section{read_route.c:}

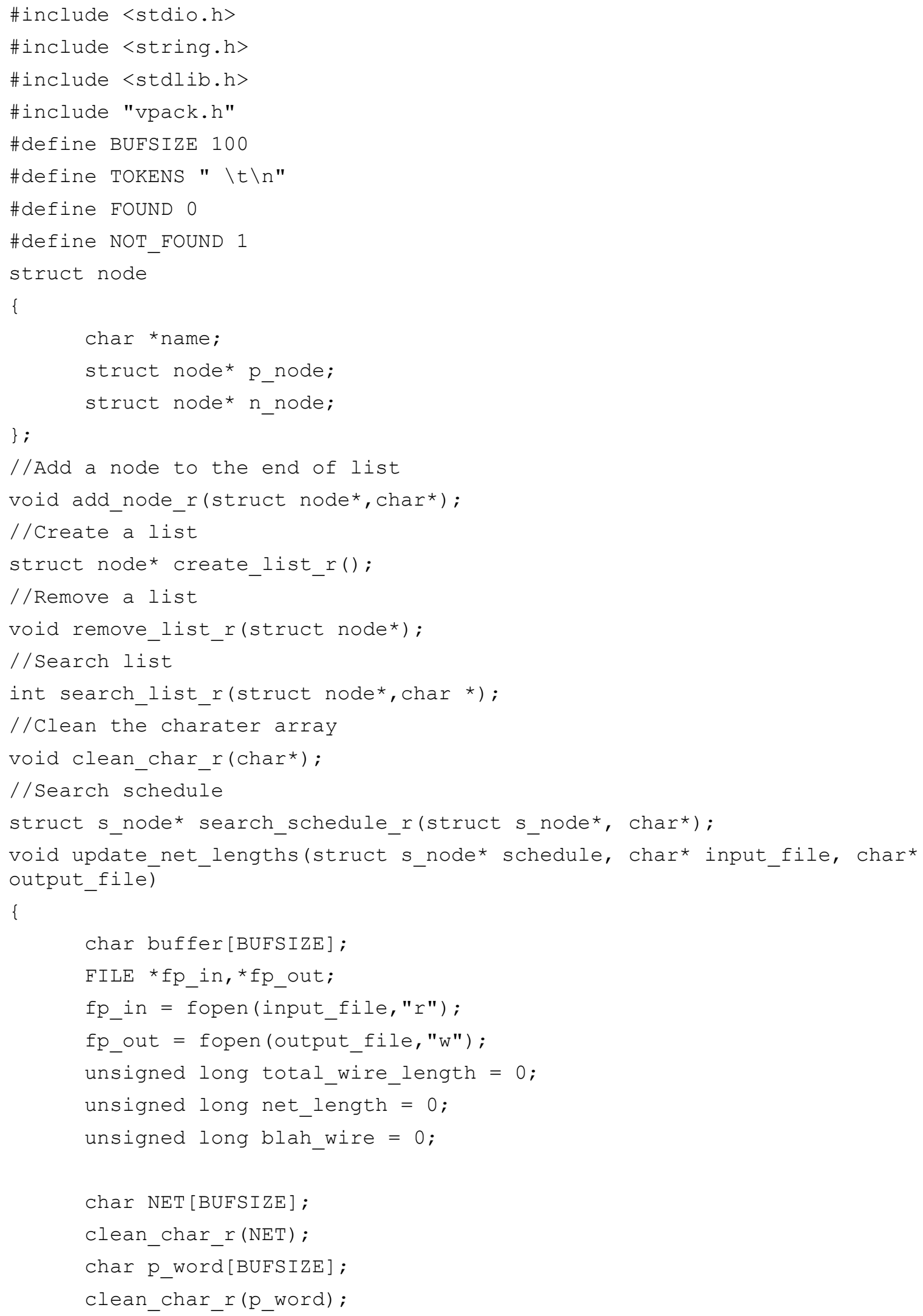




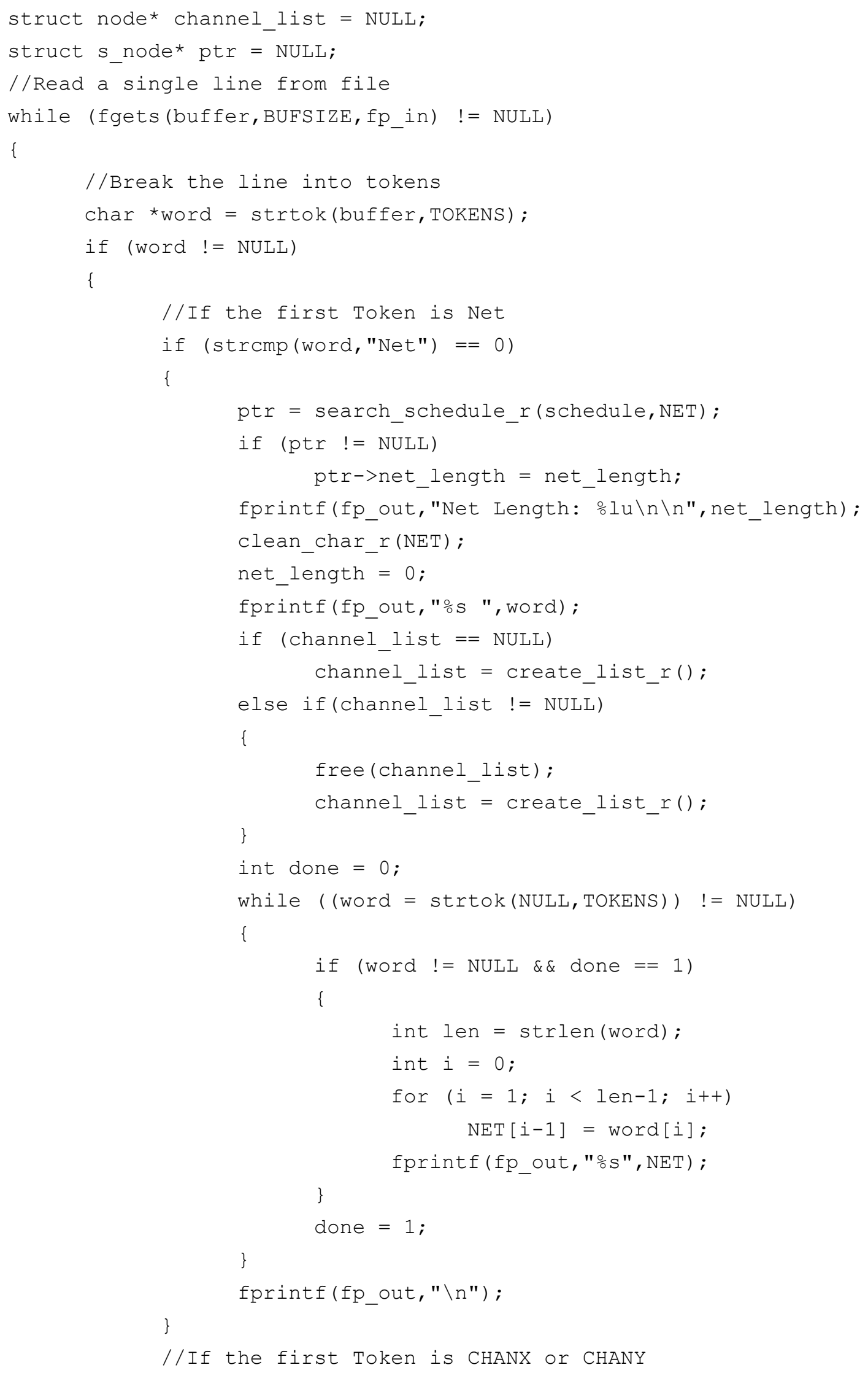


$==0)$

else if (strcmp (word, "CHANX") == 0 || strcmp (word,"CHANY")

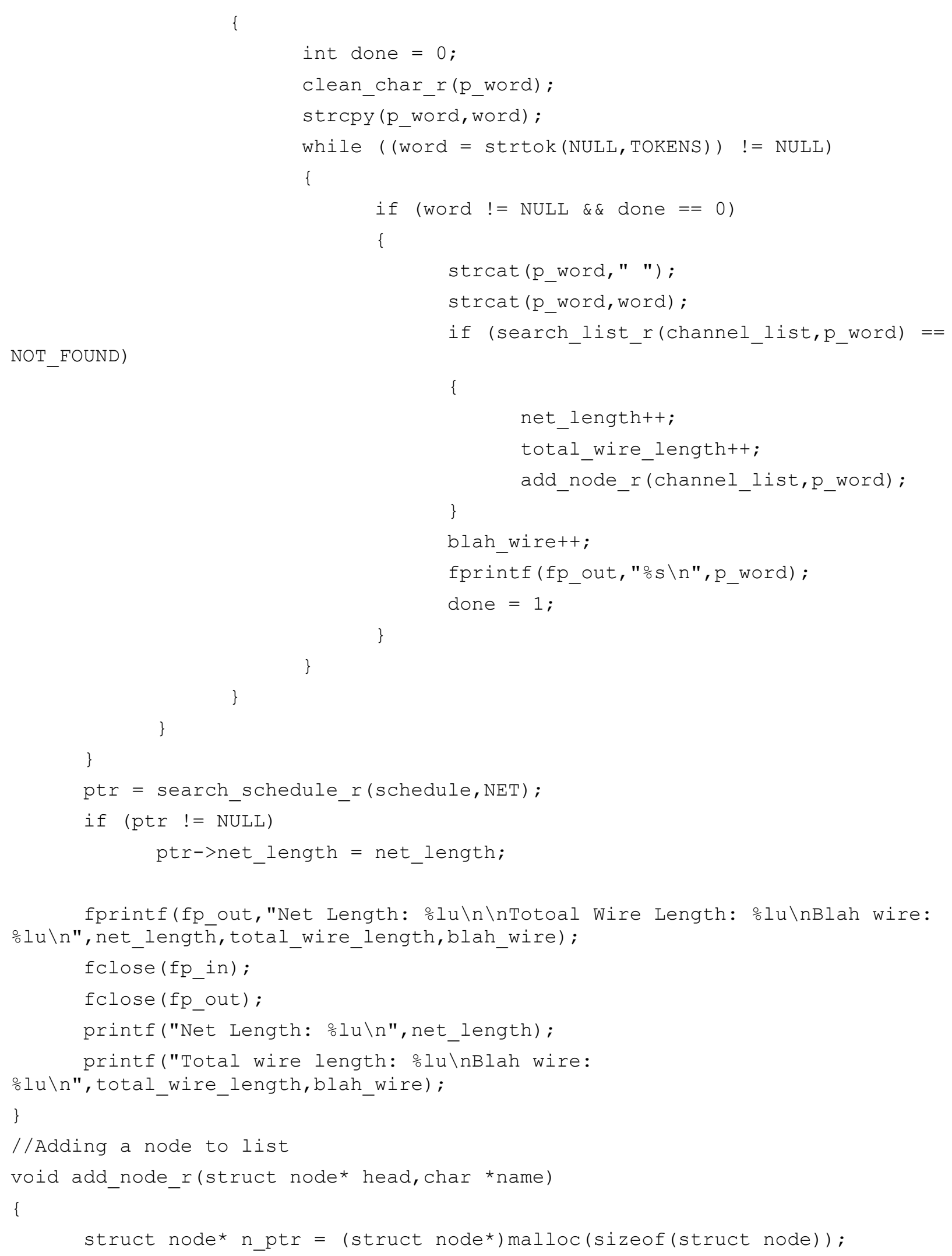




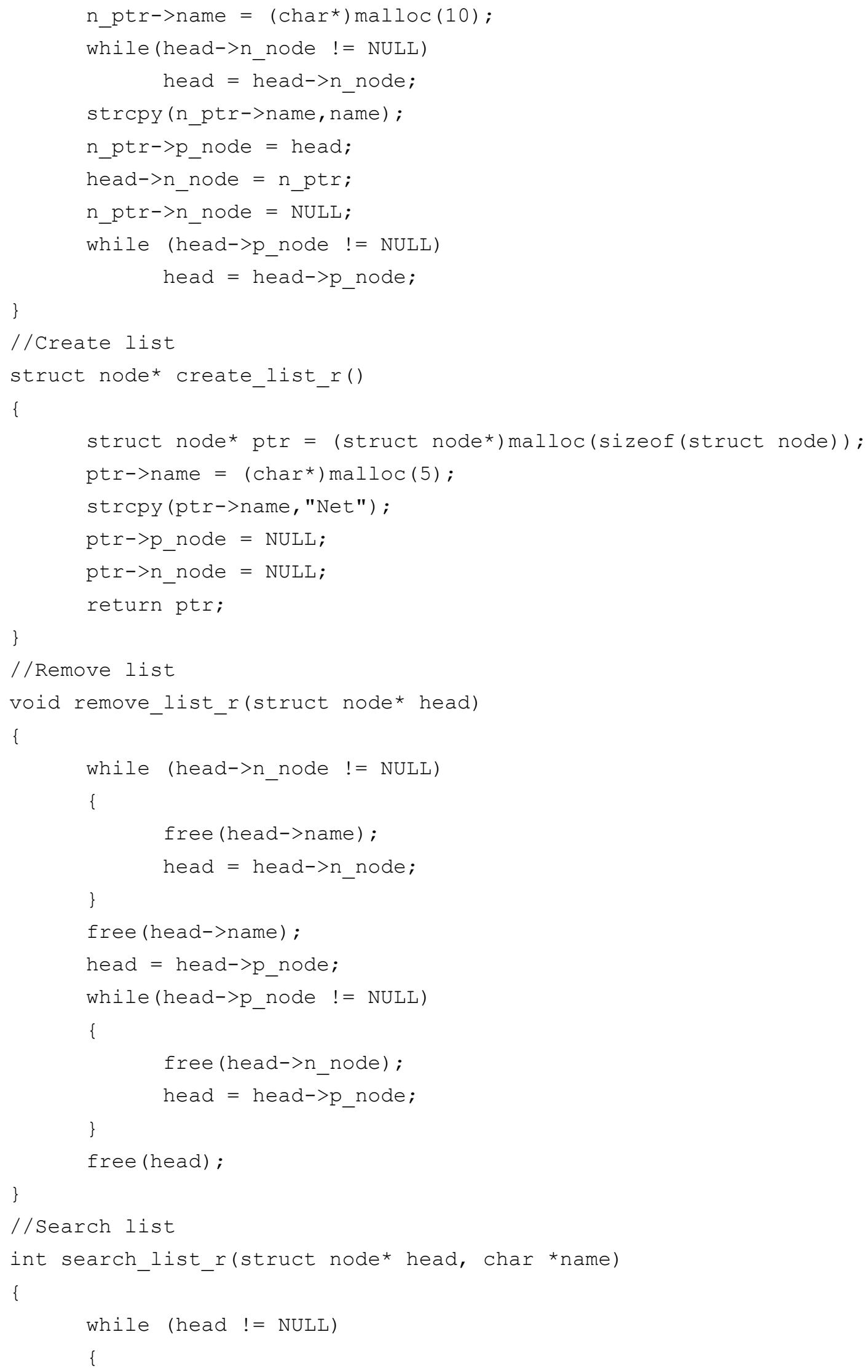




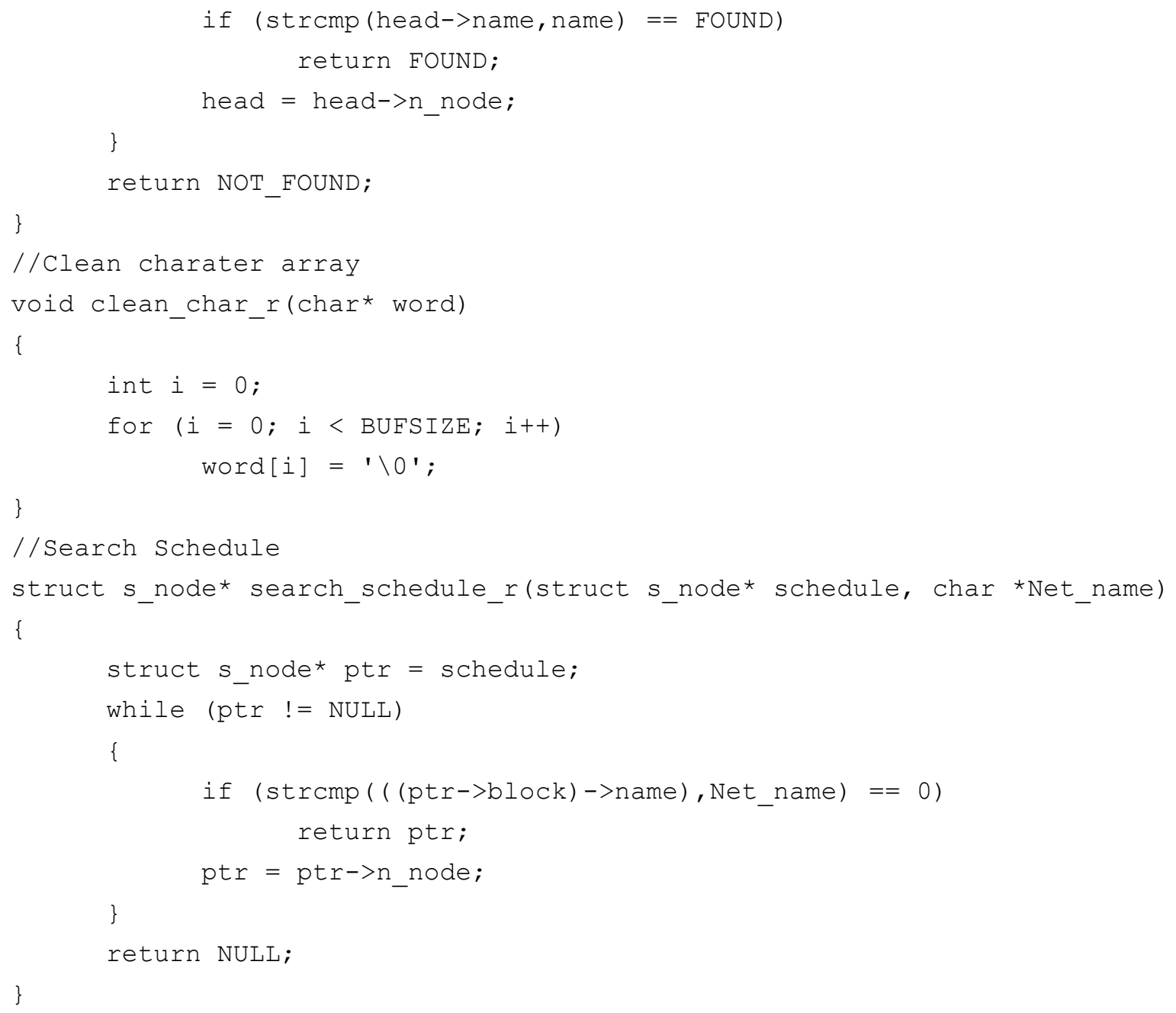




\section{H-Files:}

\section{globals.h:}

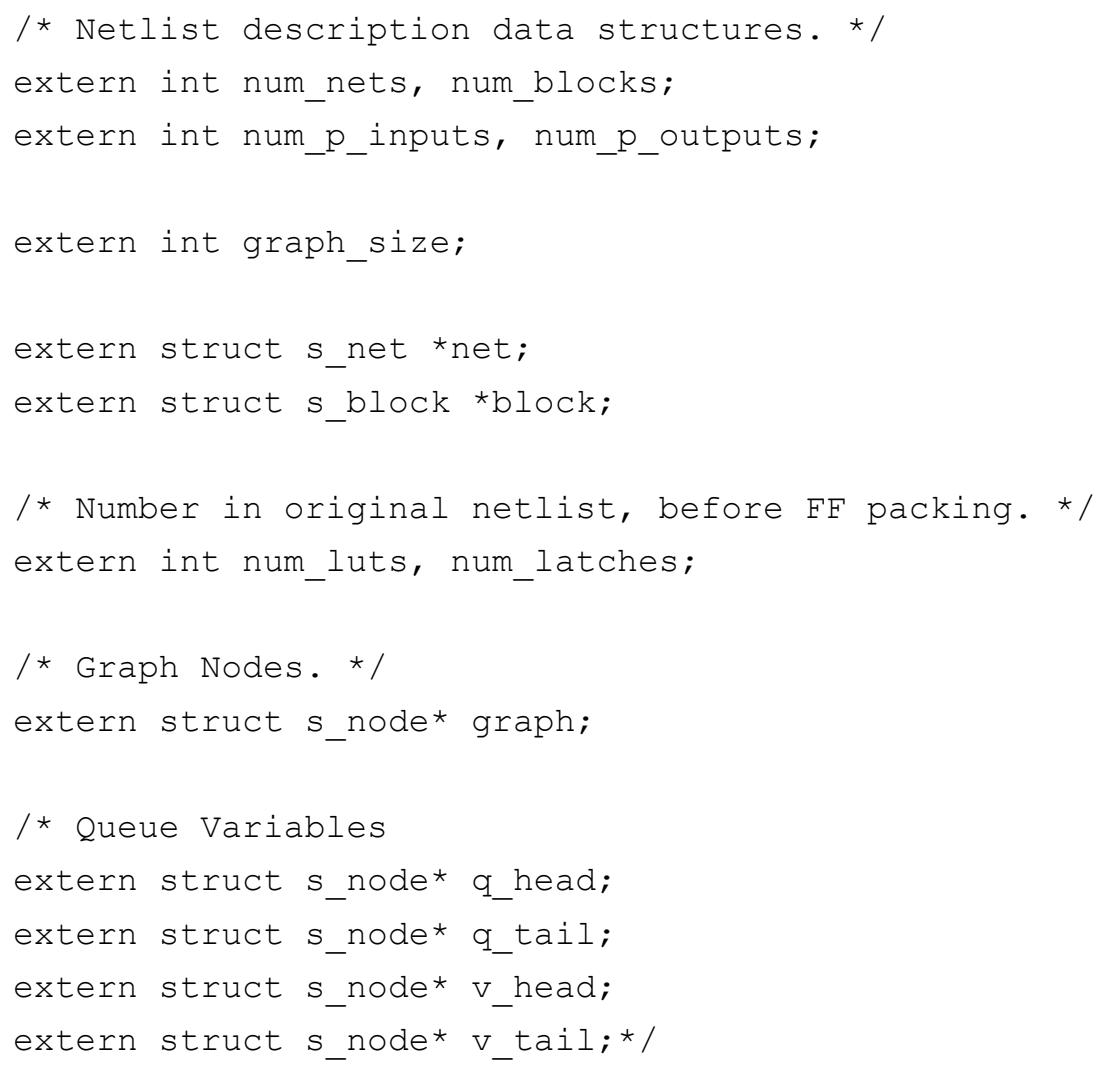

\section{graph.h:}

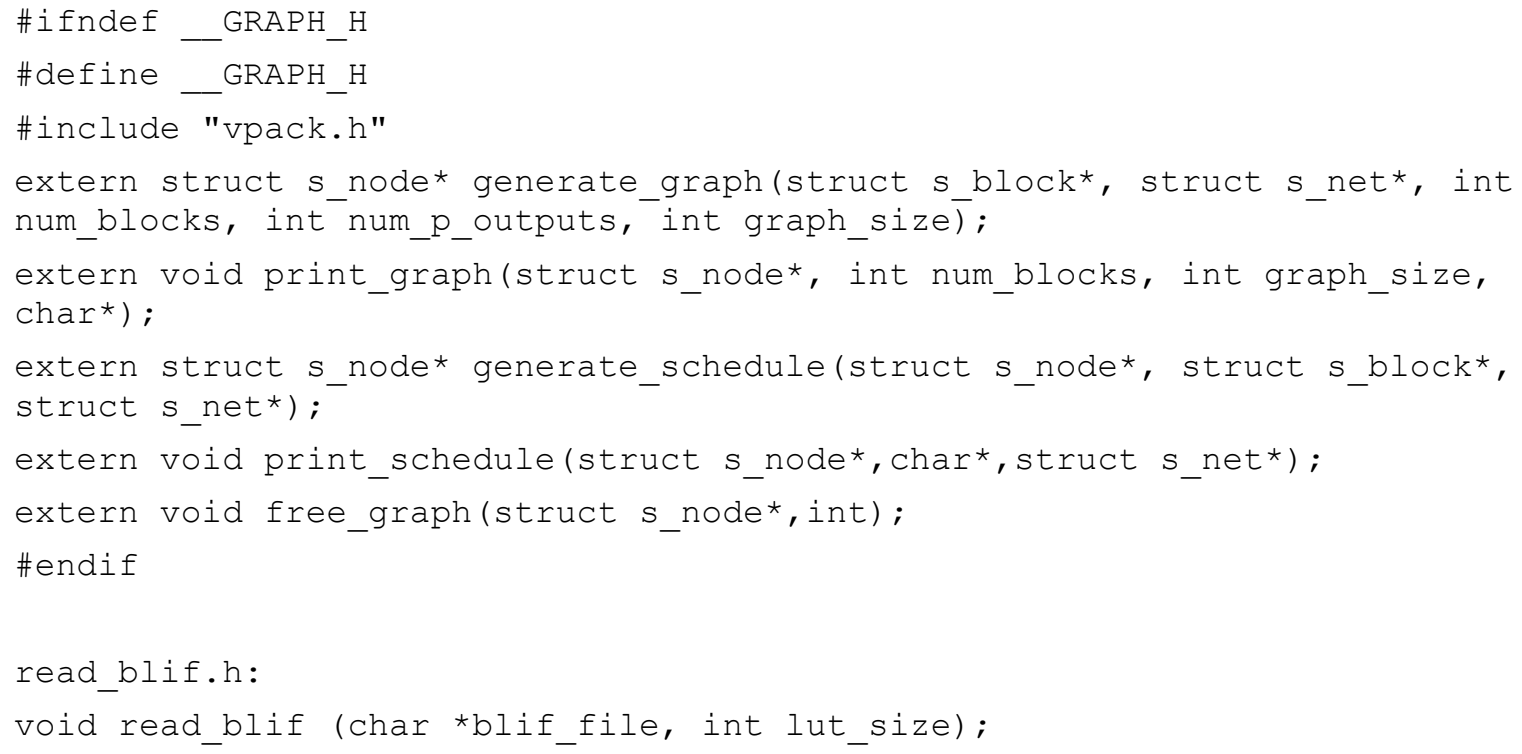




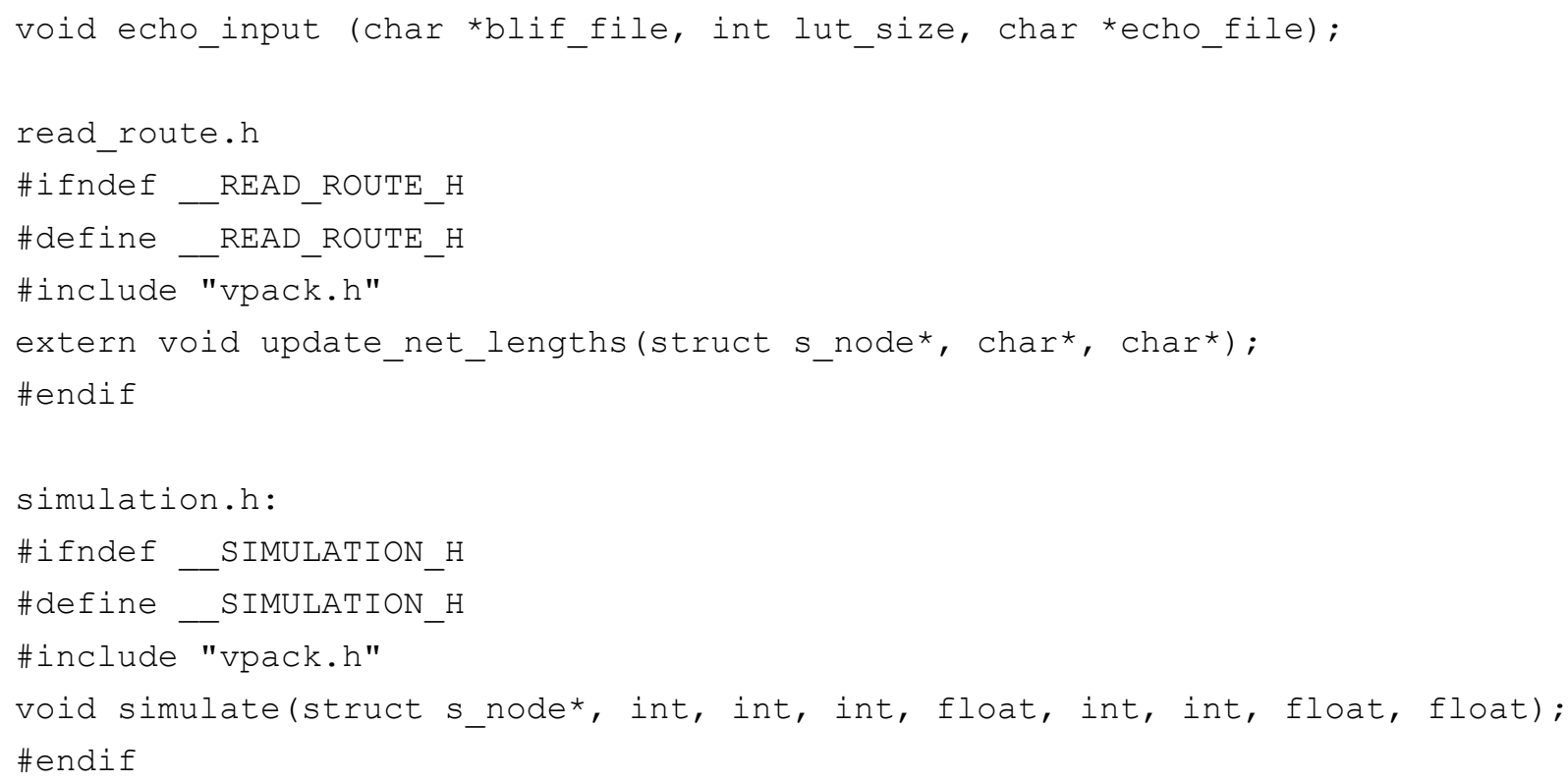

\section{util.h:}

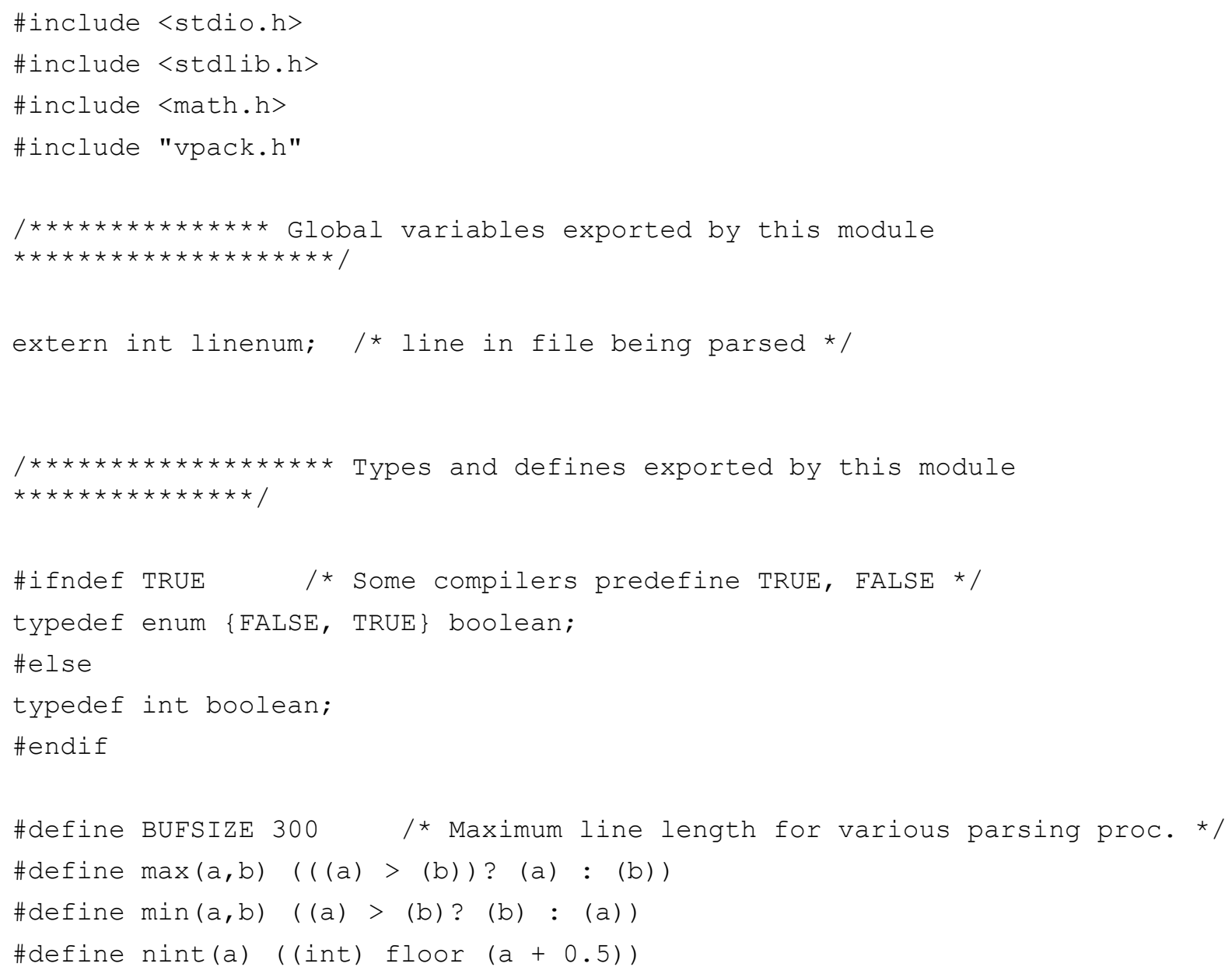




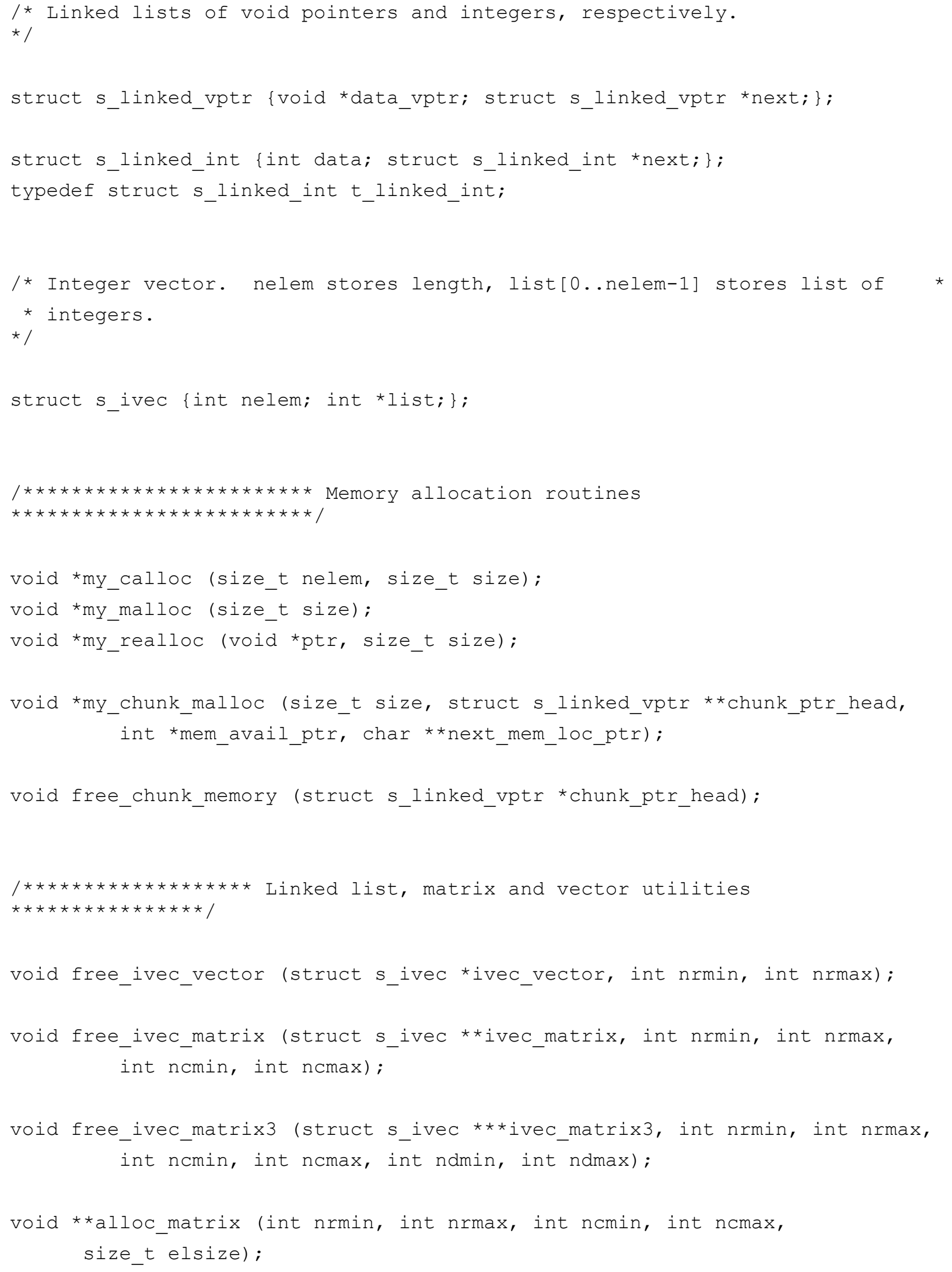




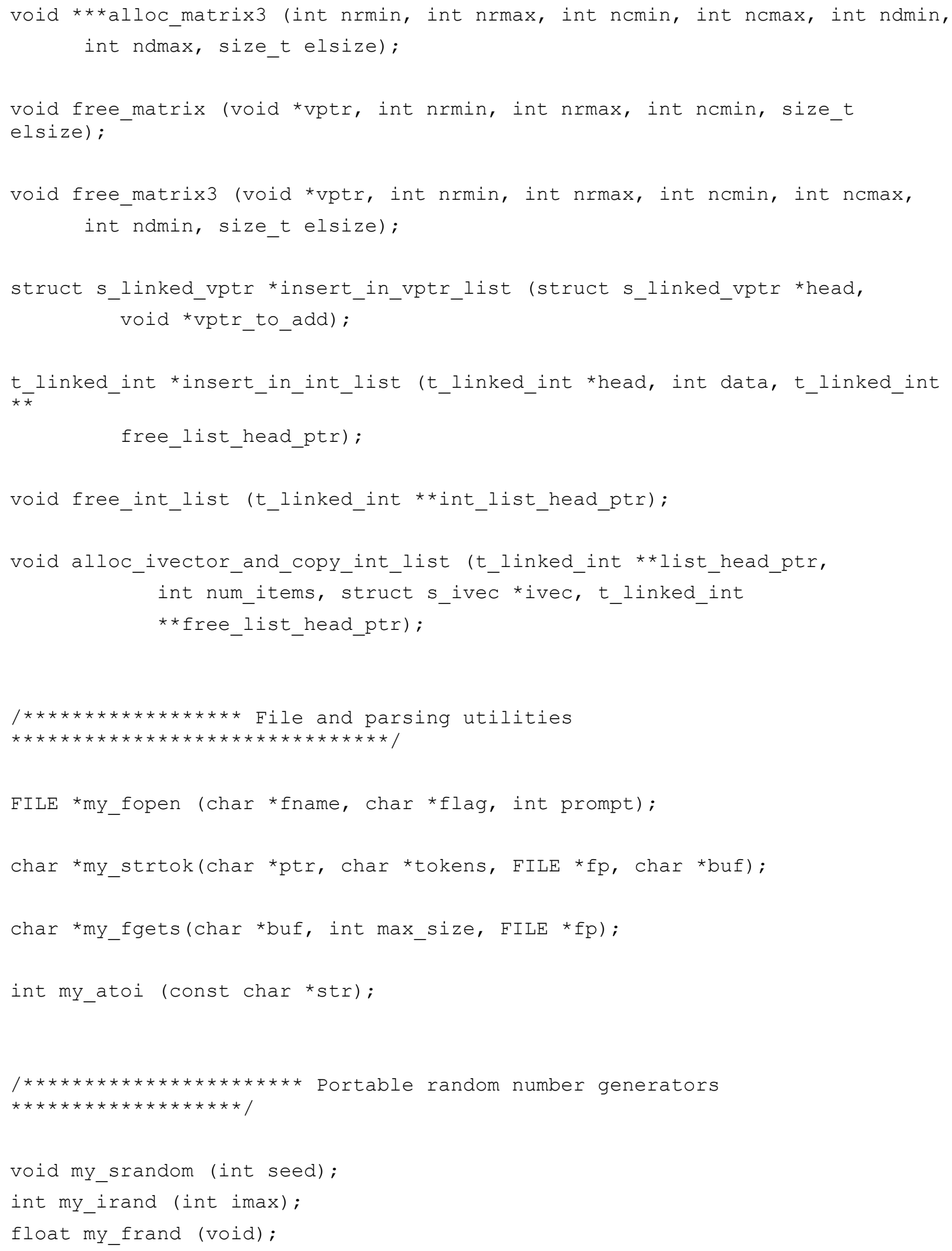




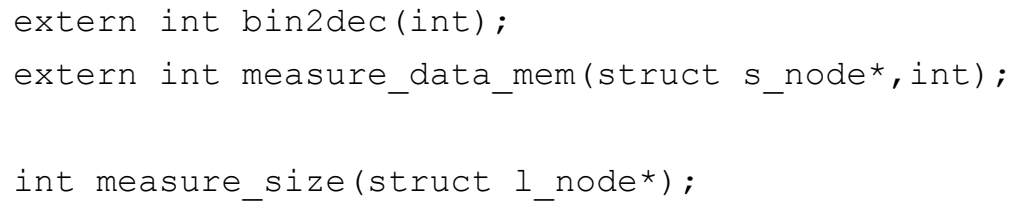

\section{vpack.h:}

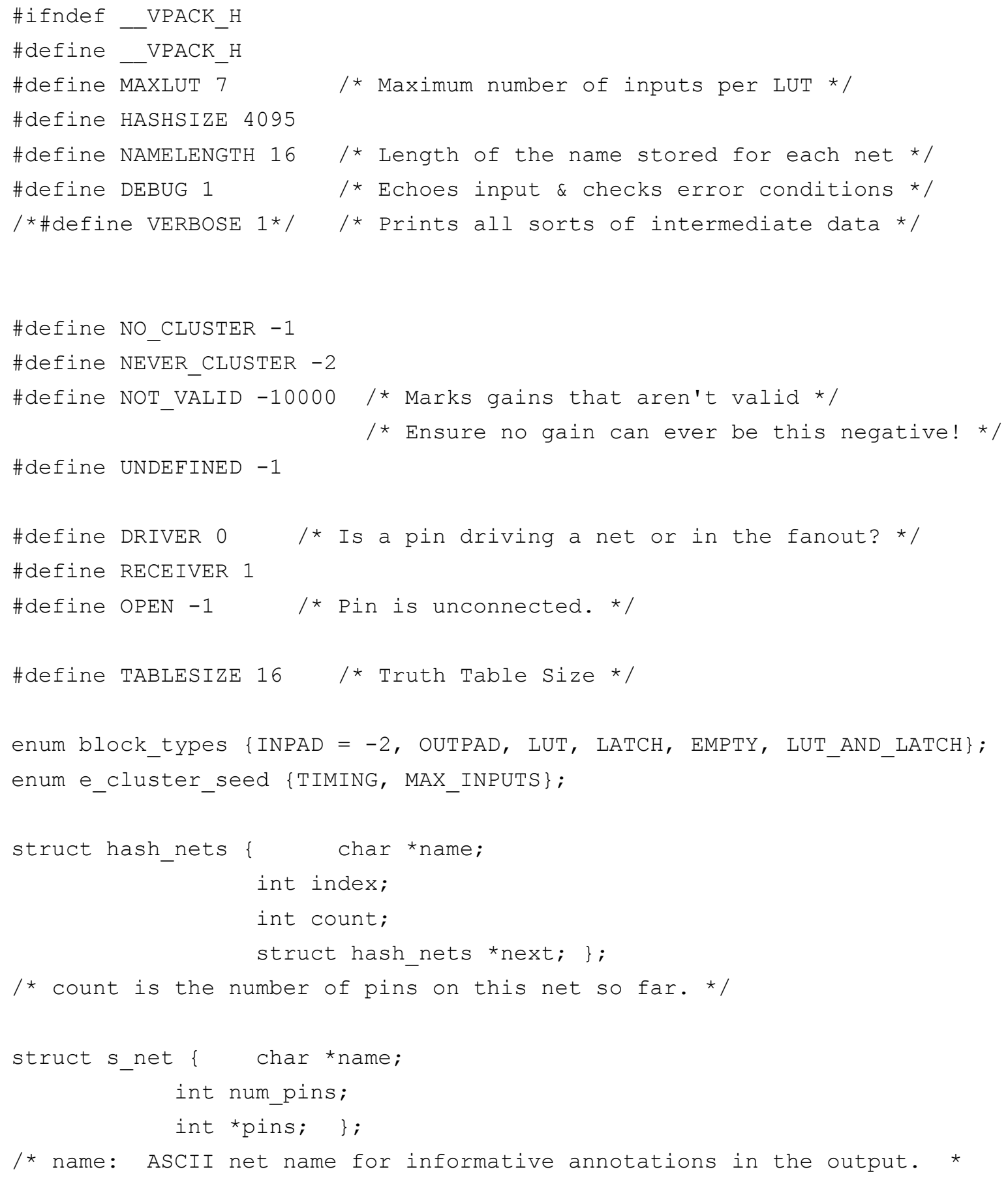




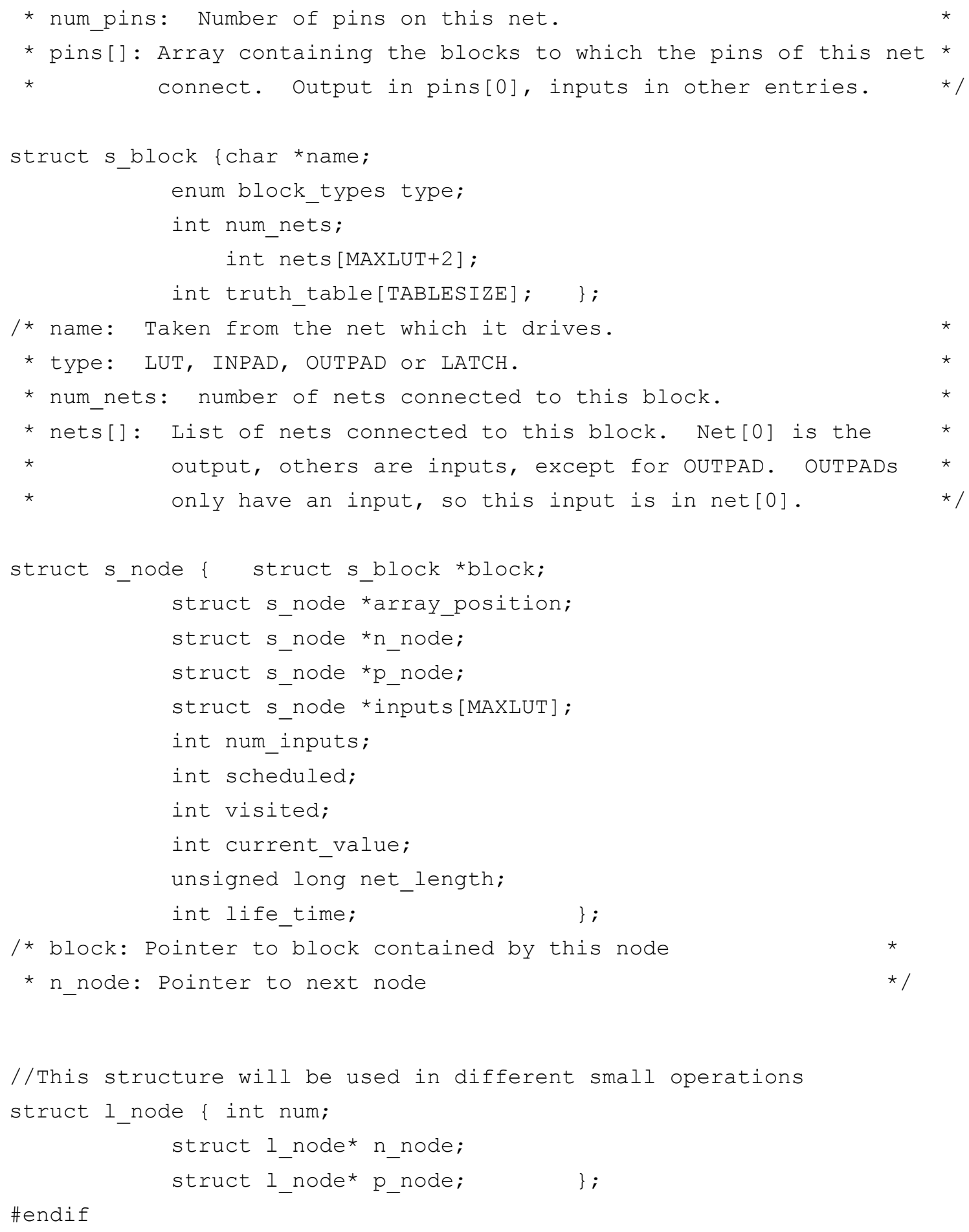




\section{Blif files:}

\section{8_bit_compressor_tree:}

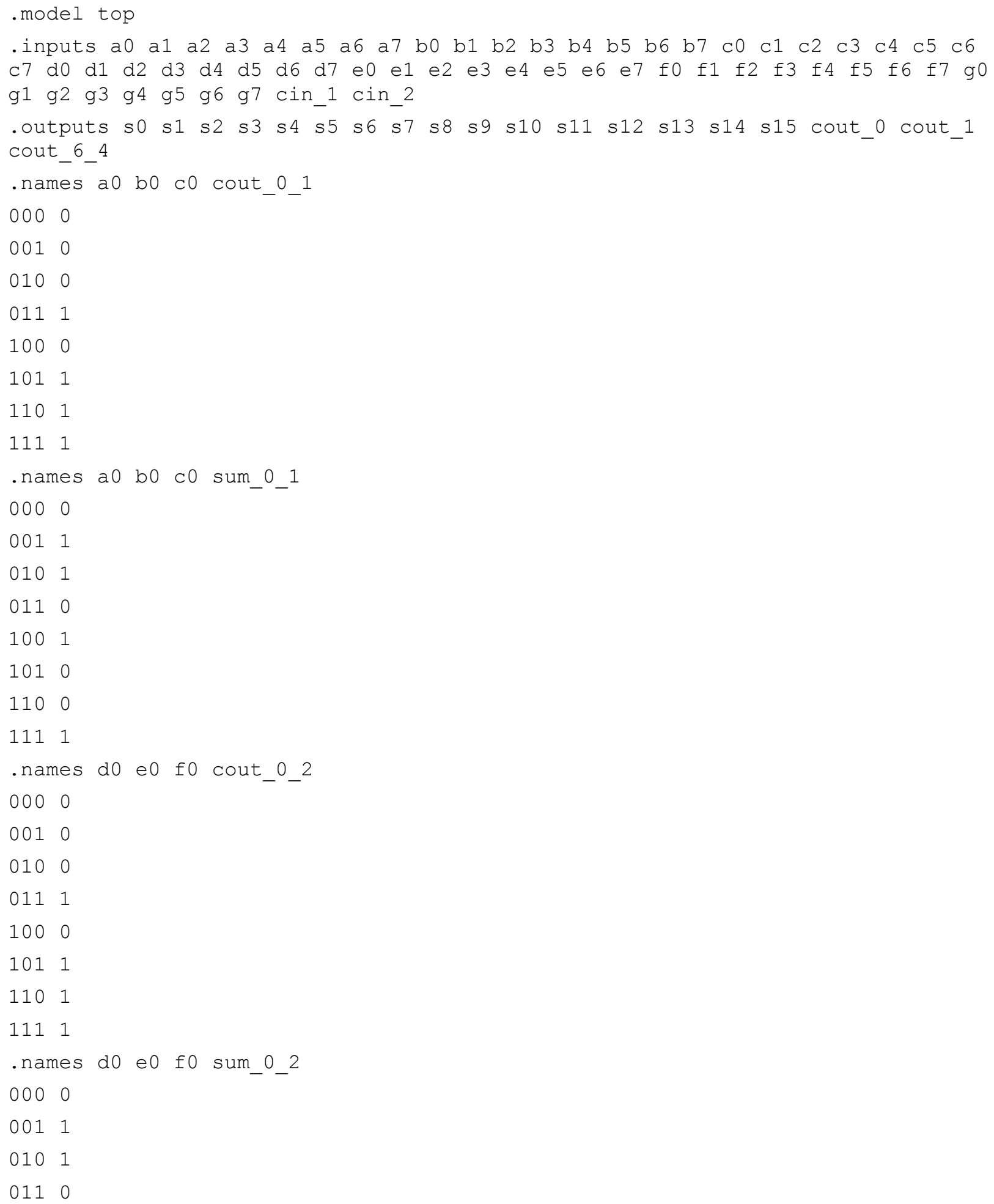




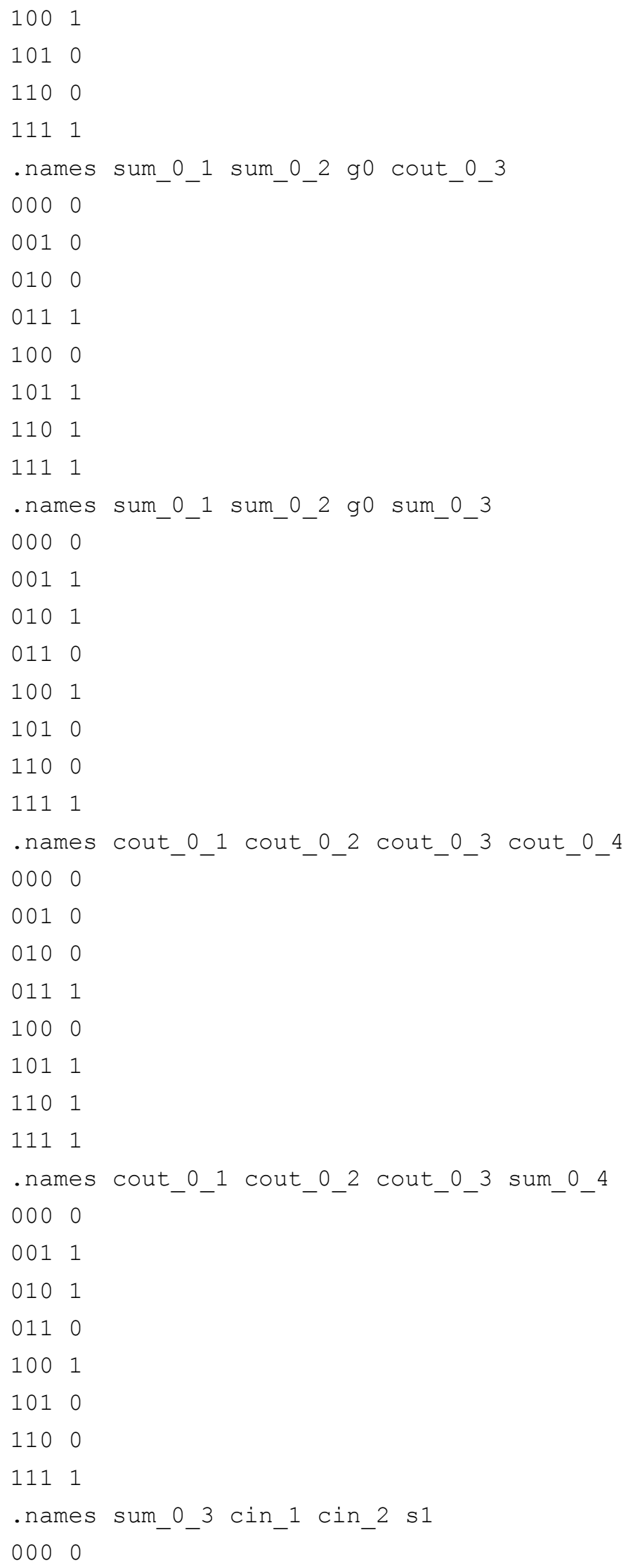




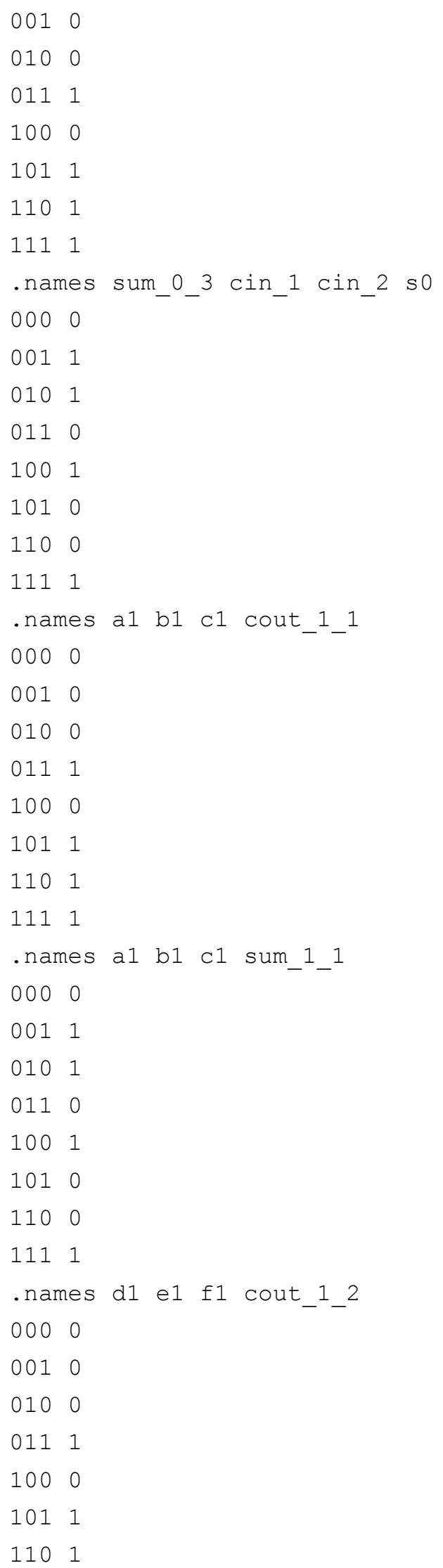


1111

- names dl el fl sum 12

0000

0011

0101

0110

1001

1010

1100

1111

. names sum_1_1 sum_1_2 g1 cout_1_3

0000

0010

0100

0111

1000

1011

1101

1111

. names sum_1_1 sum_1_2 g1 sum_1_3

0000

0011

0101

0110

1001

1010

1100

1111

. names cout_1_1 cout_1_2 cout_1_3 cout_1_4 0000

0010

0100

0111

1000

1011

1101

1111

. names cout_1 ${ }^{1}$ cout_ ${ }^{1}{ }^{2}$ cout_1 ${ }^{1} 3$ sum_1 ${ }^{4}$

0000

0011

0101

0110 


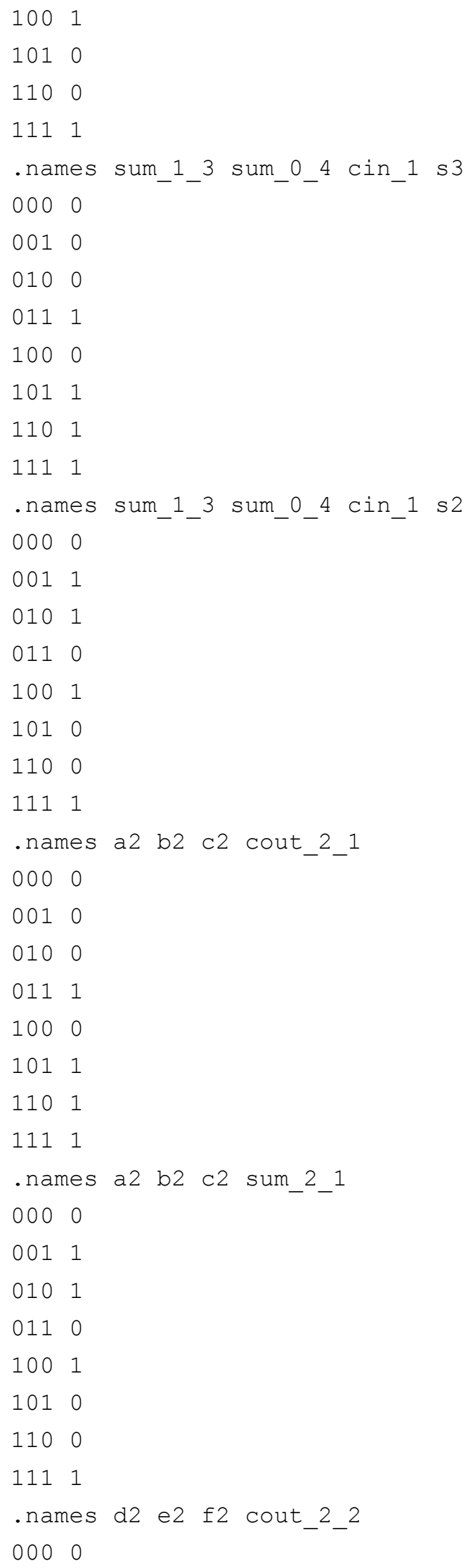




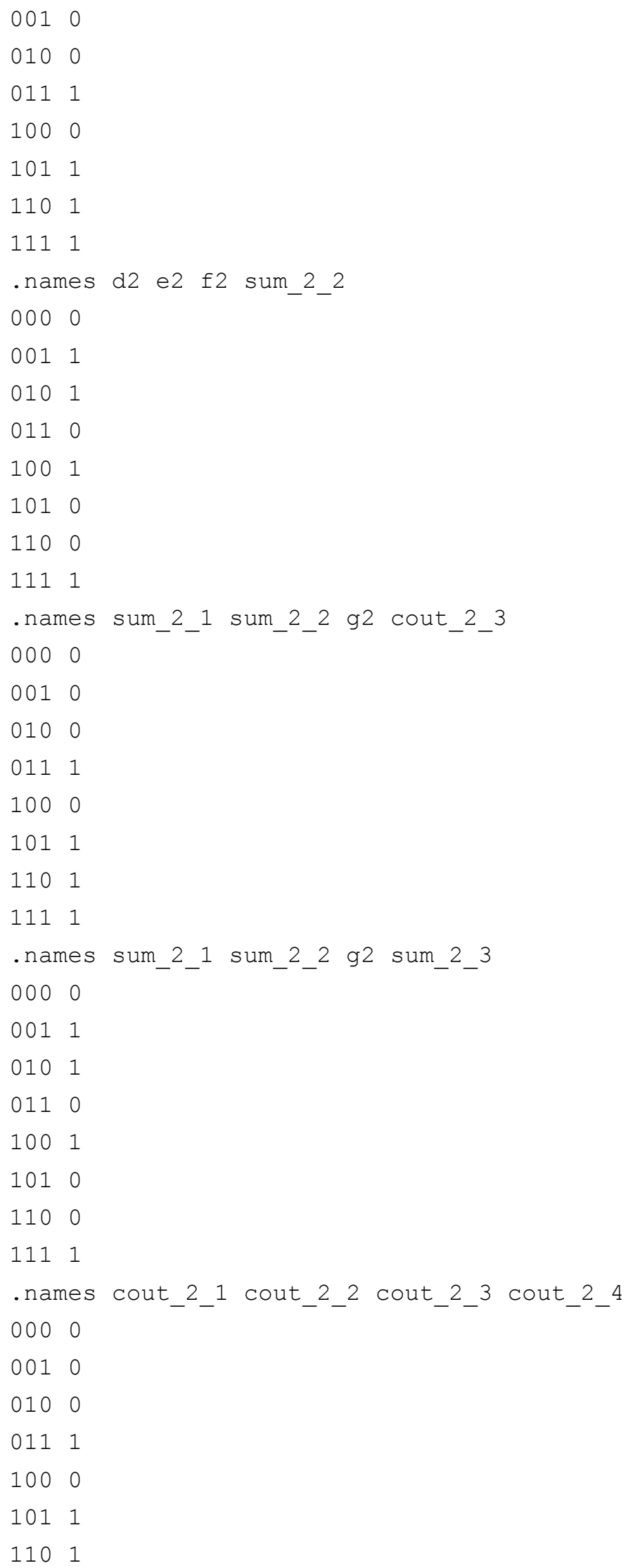




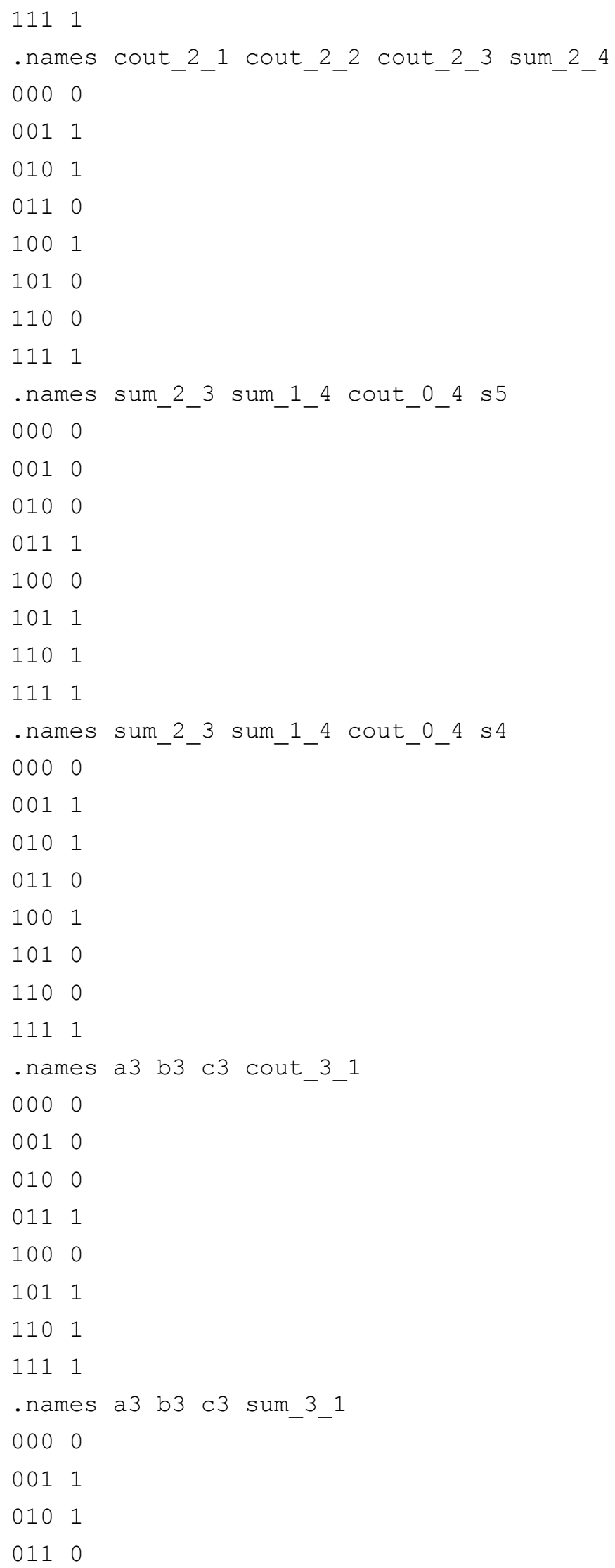


1001

1010

1100

1111

. names d3 e3 f3 cout_3_2

0000

0010

0100

0111

1000

1011

1101

1111

. names d3 e3 f3 sum_3_2

0000

0011

0101

0110

1001

1010

1100

1111

.names sum_3_1 sum_3_2 g3 cout_3_3

0000

0010

0100

0111

1000

1011

1101

1111

. names sum_3_1 sum_3_2 g3 sum_3_3

0000

0011

0101

0110

1001

1010

1100

1111

. names cout_3_1 cout_3_2 cout_3_3 cout_3 ${ }^{3} 4$

0000 


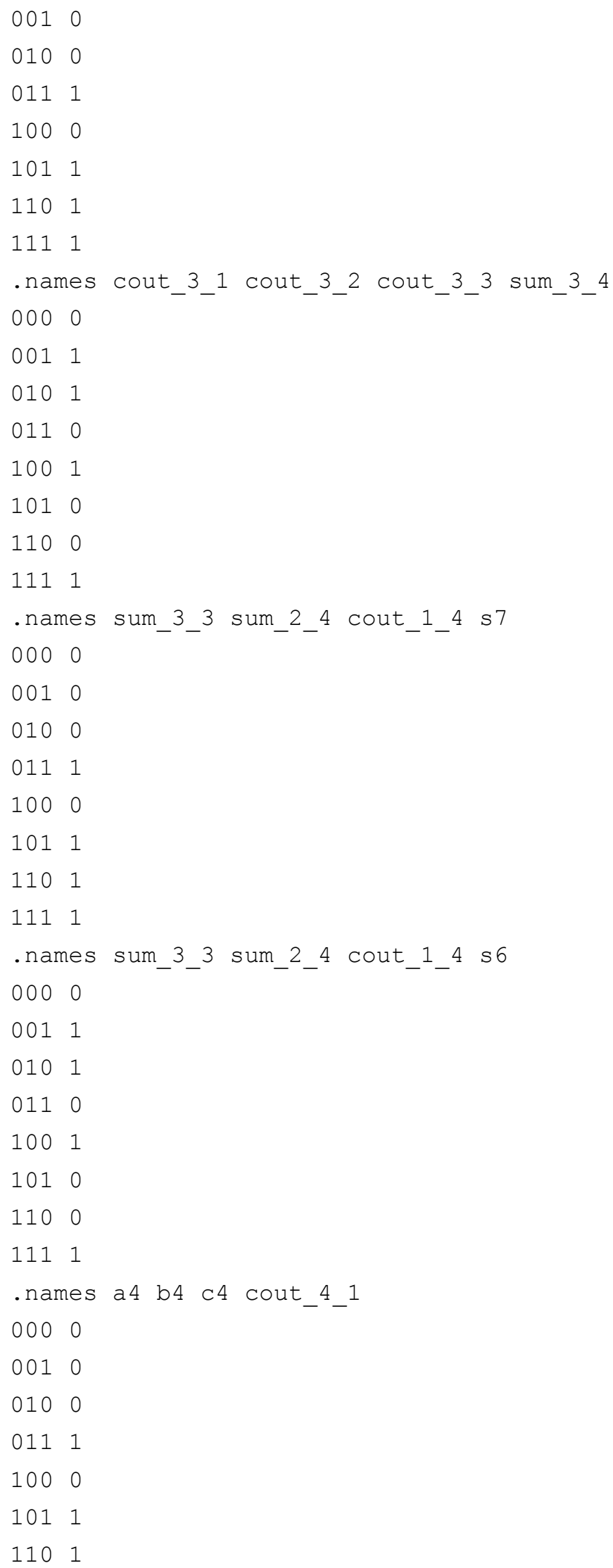


1111

- names a 4 b4 c4 sum_4_1

0000

0011

0101

0110

1001

1010

1100

1111

. names d4 e4 f4 cout_ $4{ }^{2}$

0000

0010

0100

0111

1000

1011

1101

1111

. names d4 e4 f4 sum_4_2

0000

0011

0101

0110

1001

1010

1100

1111

. names sum_4_1 sum_4_2 g4 cout_4_3

0000

0010

0100

0111

1000

1011

1101

1111

. names sum_4_1 sum_4 2 g4 sum_4_3

0000

0011

0101

0110 


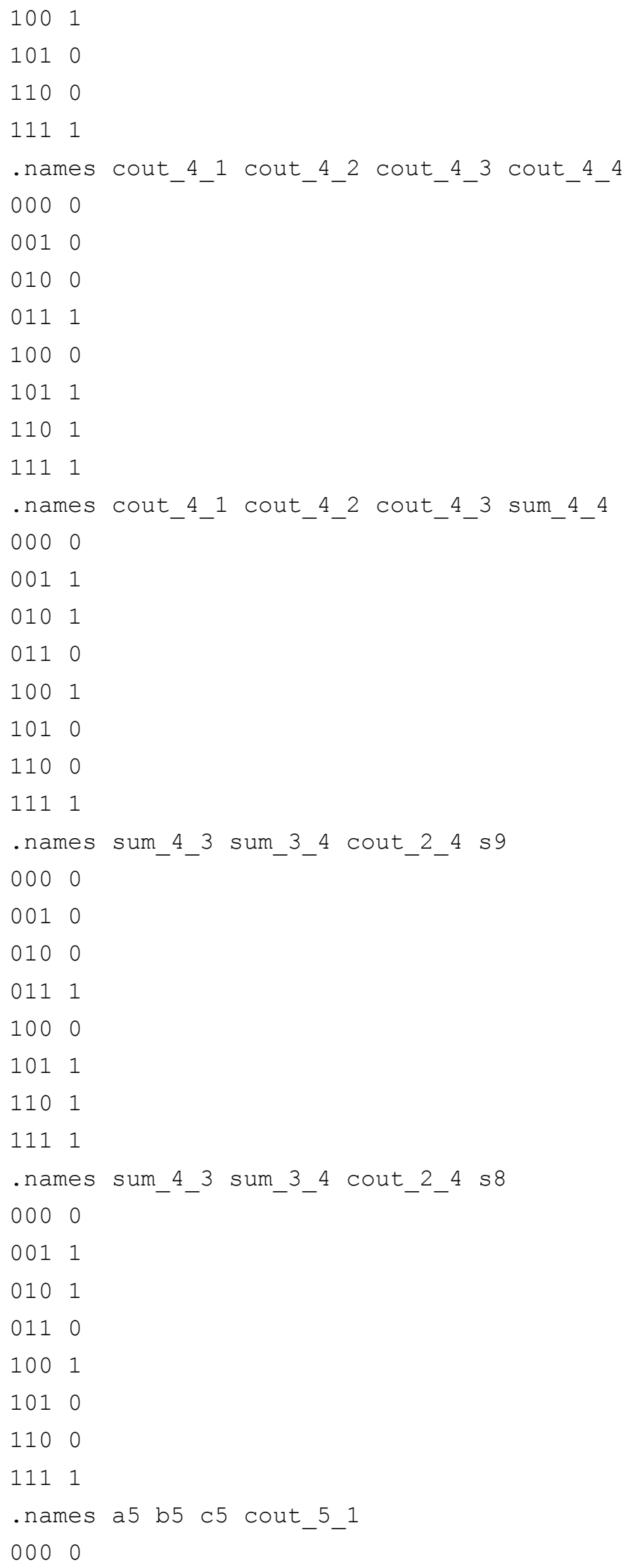




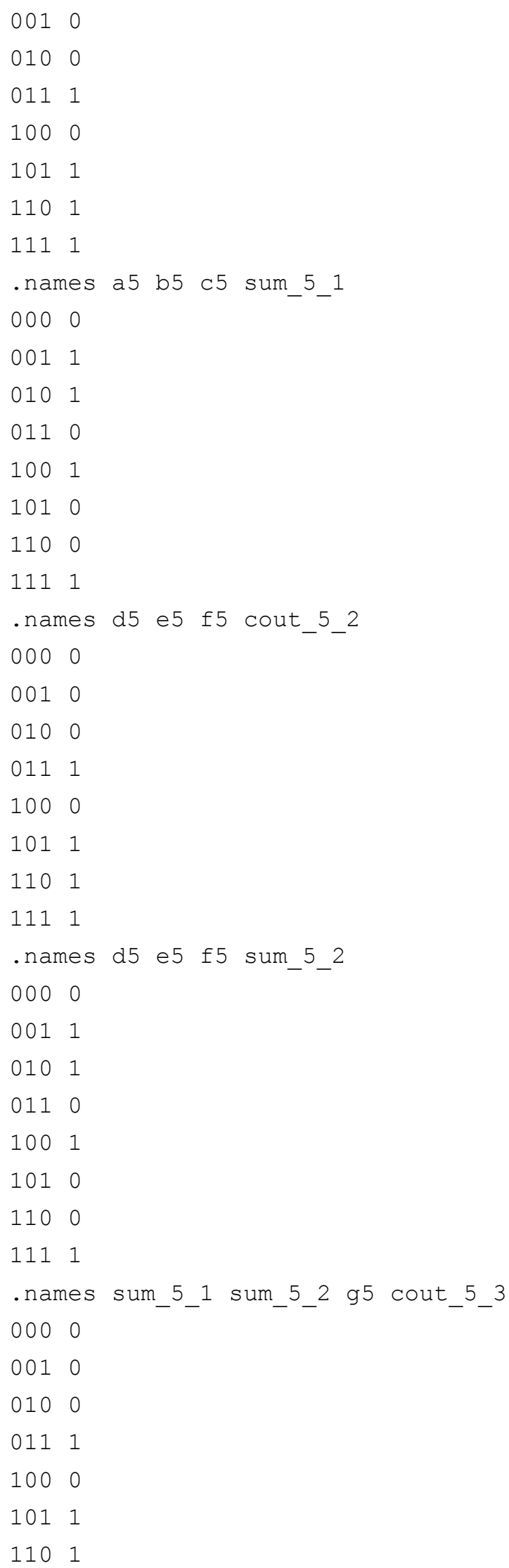




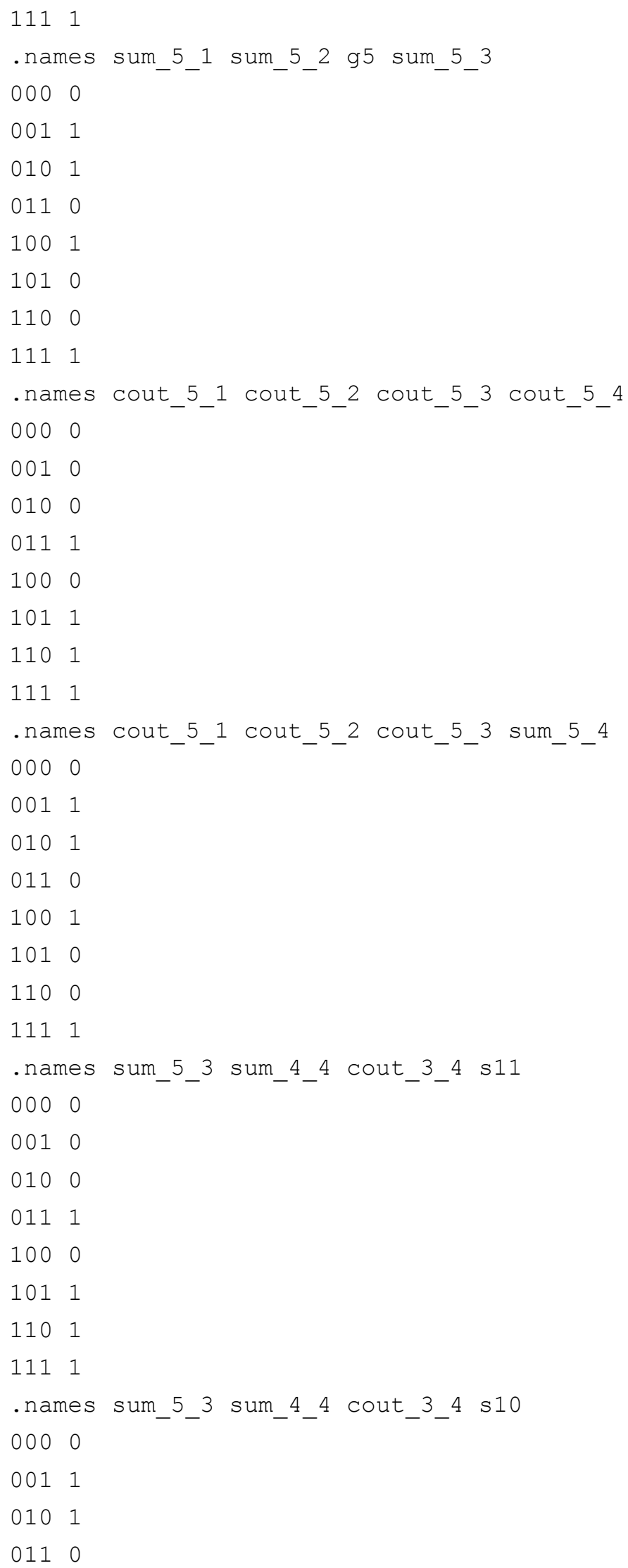


1001

1010

1100

1111

. names a 6 b6 c6 cout 6 _ 1

0000

0010

0100

0111

1000

1011

1101

1111

. names a 6 b6 c6 sum_6_1

0000

0011

0101

0110

1001

1010

1100

111

. names d 6 e 6 f6 cout_ $6{ }^{2}$

0000

0010

0100

0111

1000

1011

1101

111

- names d6 e6 f6 sum_6_2

0000

0011

0101

0110

1001

1010

1100

1111

.names sum_6_1 sum_6_2 g6 cout_6_3

0000 


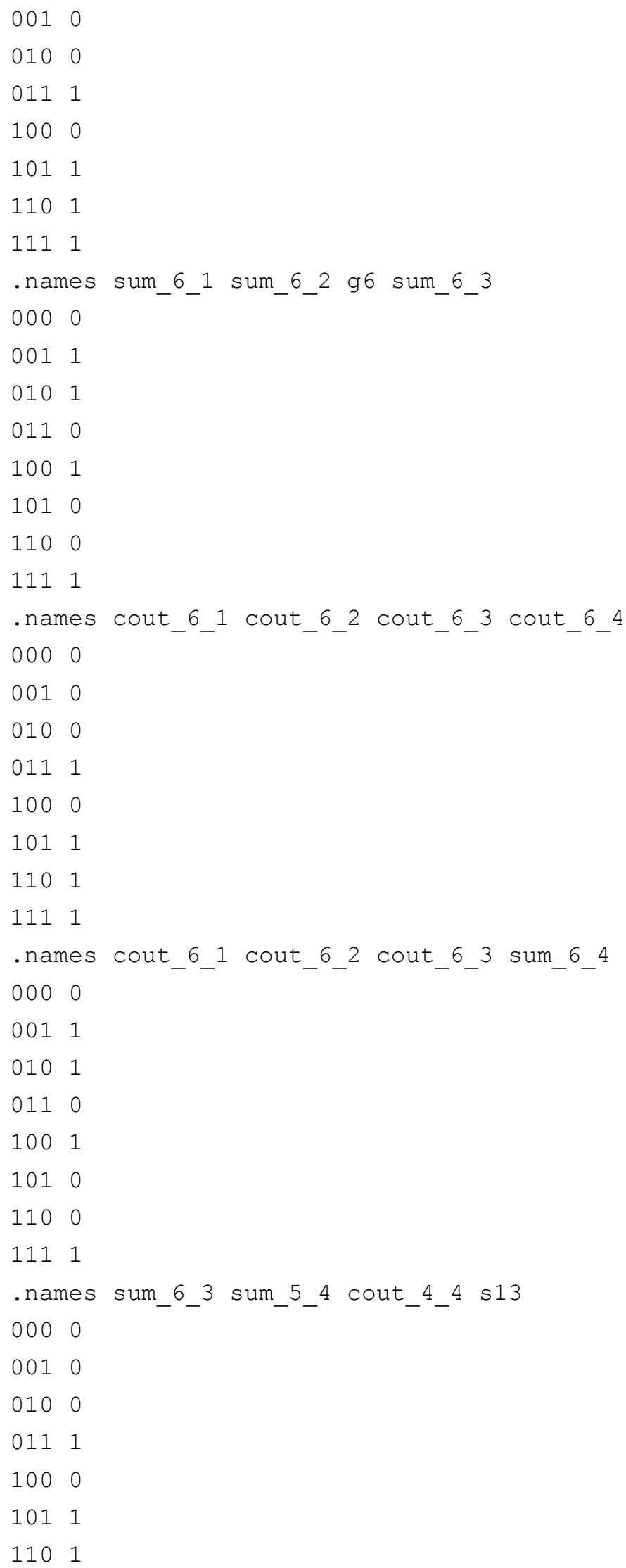




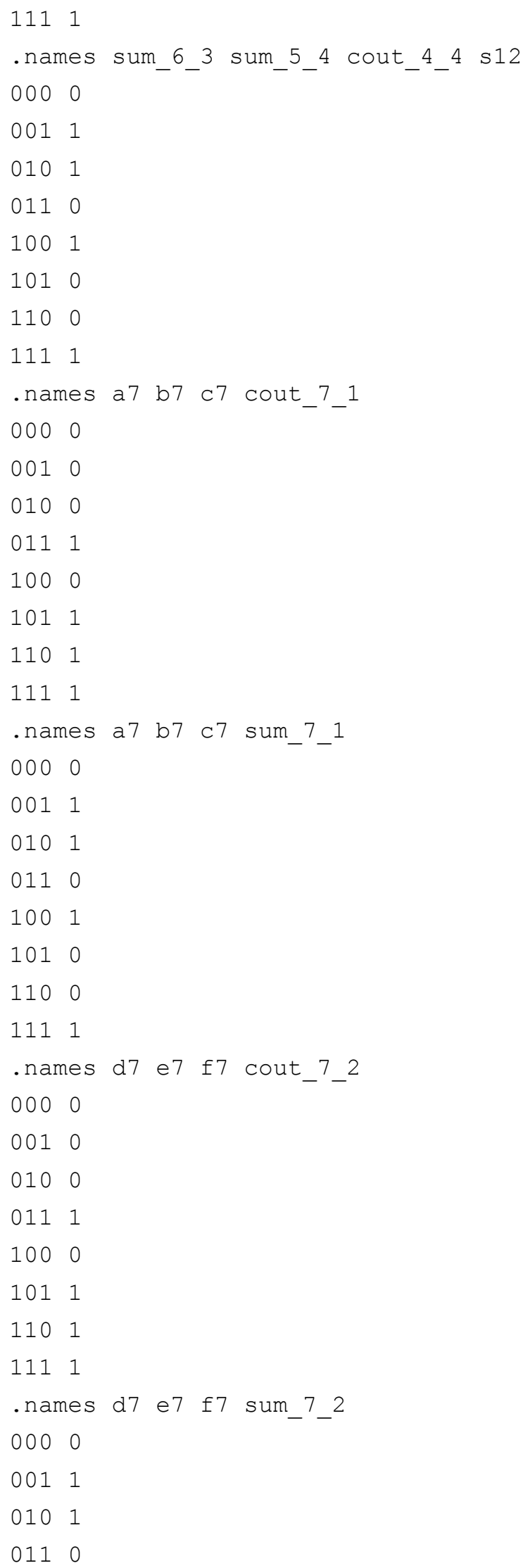




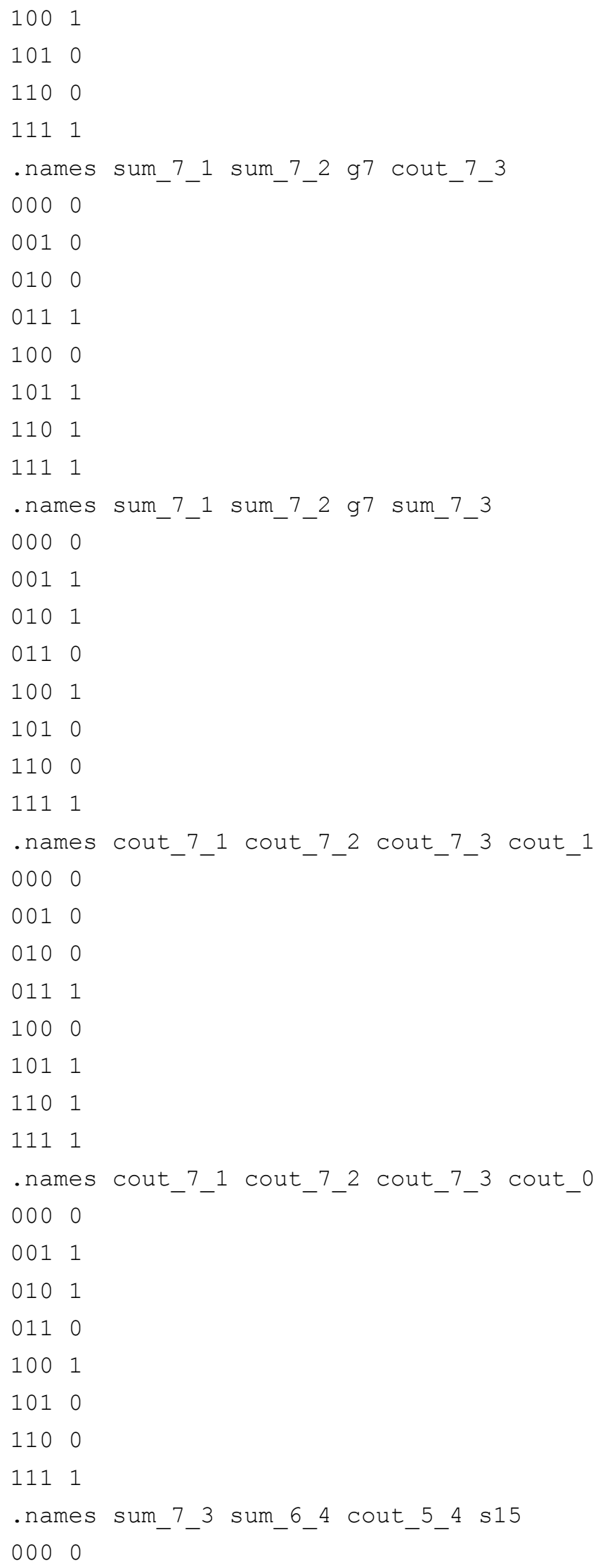




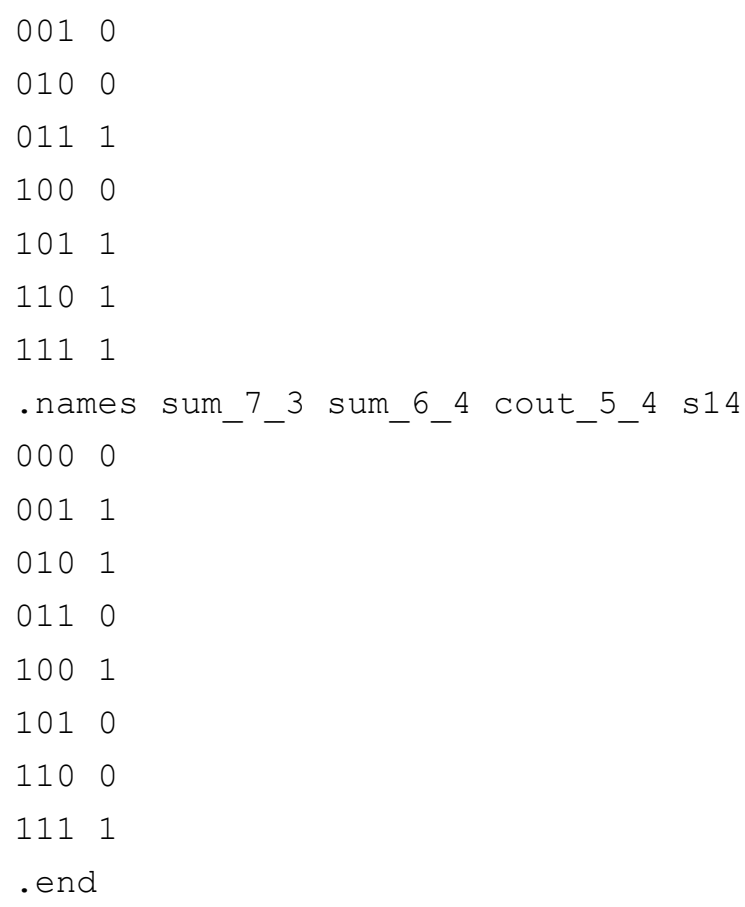




\section{8_Full_Adders:}

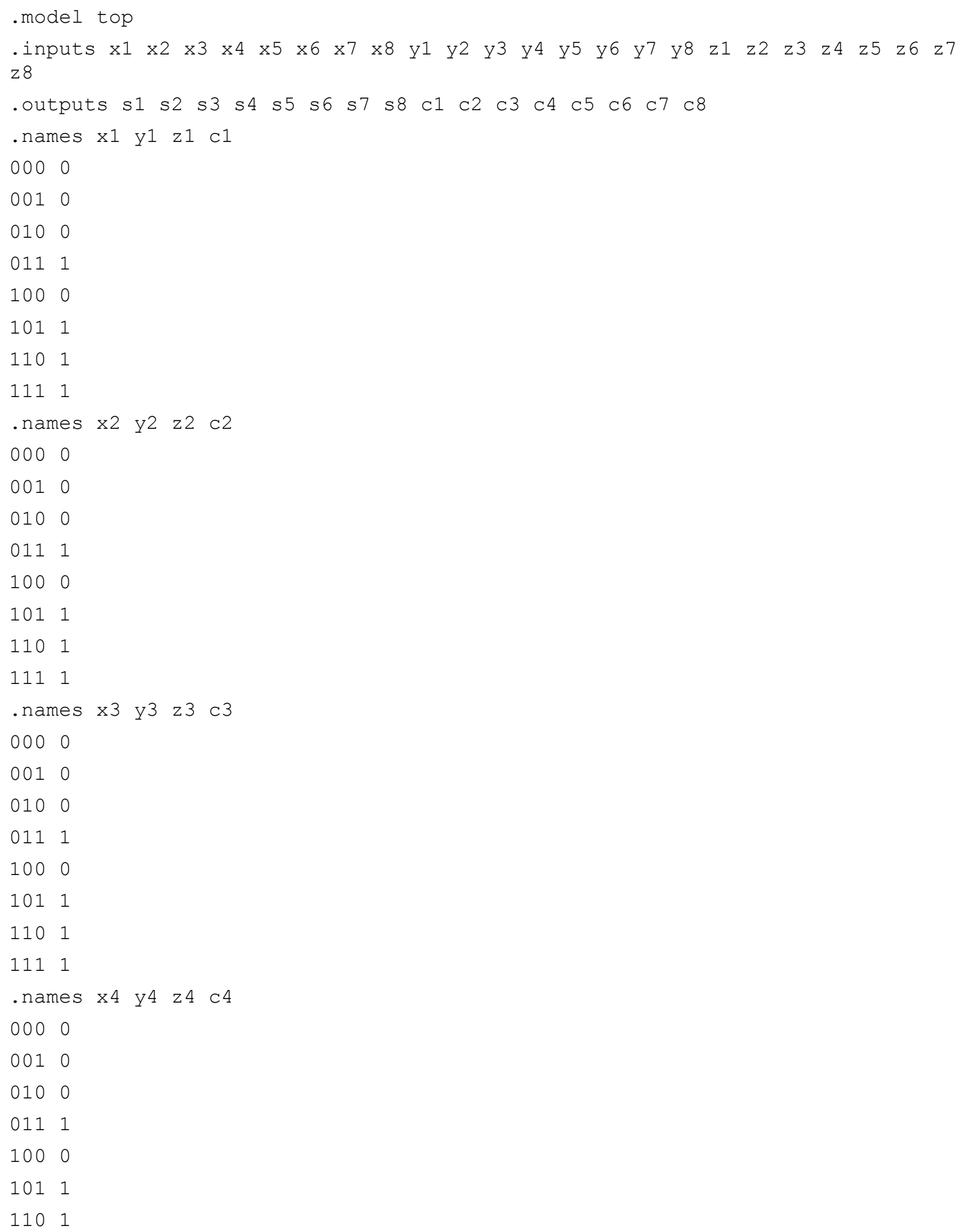


1111

.names x 5 y5 z5 c5

0000

0010

0100

0111

1000

1011

1101

1111

. names x6 y6 z6 c6

0000

0010

0100

0111

1000

1011

1101

1111

.names $x 7$ y $7 \quad$ z $7 \quad$ c7

0000

0010

0100

0111

1000

1011

1101

1111

.names x8 y8 z8 c8

0000

0010

0100

0111

1000

1011

1101

1111

. names x1 y1 z1 s1

0000

0011

0101

0110 


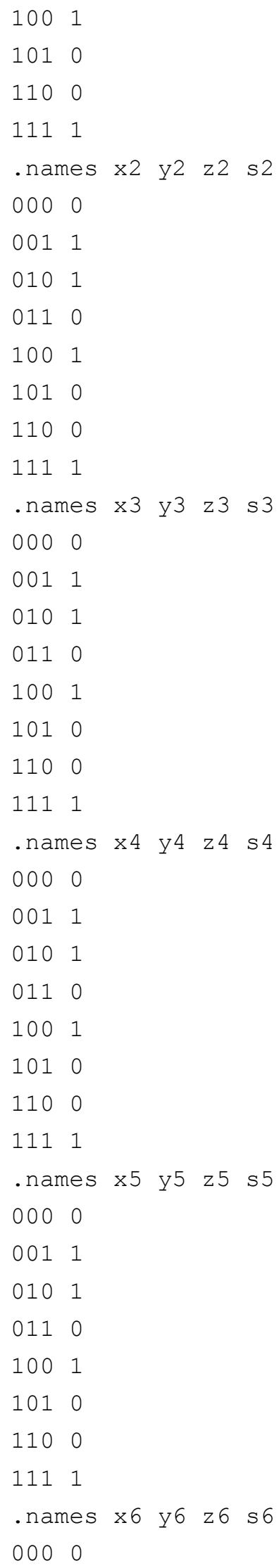




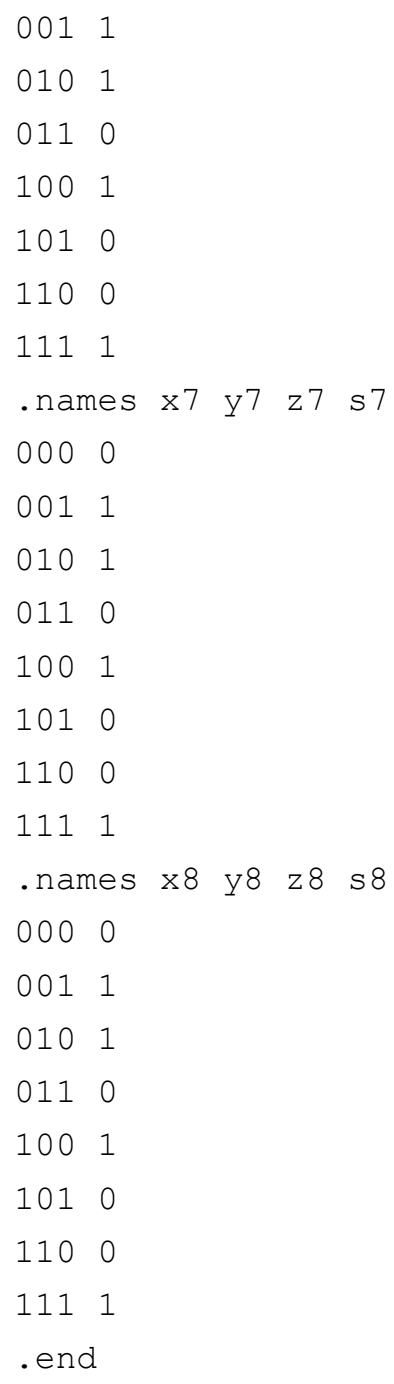

\section{Full Adder:}

-model top

. inputs a b c

-outputs sum carry

- names a b c carry

0000

0010

0100

0111

1000

1011

1101

1111

- names a b c sum 0000 
0011

0101

0110

1001

1010

1100

1111

.end

\section{Makefile:}

\# This makefile is written for gcc running under SLinux 6.0 on $x 86$ arch.

\# To compile T-VPack on other systems, you may have to change:

\# (1) CC to the name of your C compiler.

\# (2) OPT_FLAGS should be changed to whatever options turn on maximum

\# optimization in your compiler.

$\mathrm{CC}=\mathrm{gCC}$

\#CC = purify gCC

$\# C C=g++$

\# Overly strict flags line below. Lots of useless warnings, but can

\# let you look for redudant declarations.

\# To avoid redundant declarations here I use -D__STDC instead of

\# -D_USE_FIXED_PROTOTYPES, but that means some prototypes are missing.

\#FLAGS = -Wall -Wpointer-arith -Wcast-qual -Wstrict-prototypes -O -D_STDC

-ansi -pedantic -Wredundant-decls -Wmissing-prototypes -Wshadow -Wcast-align

-D_POSIX_SOURCE

\#Flags to be passed to the compiler. First is for strict warnings,

\#second for interactive debugging and third for optimization.

\#-D_POSIX_SOURCE stops extra declarations from being included in math.h

\#and causing -Wshadow to complain about conflicts with yl in math.h

\# (Bessel function 1 of the second kind) 


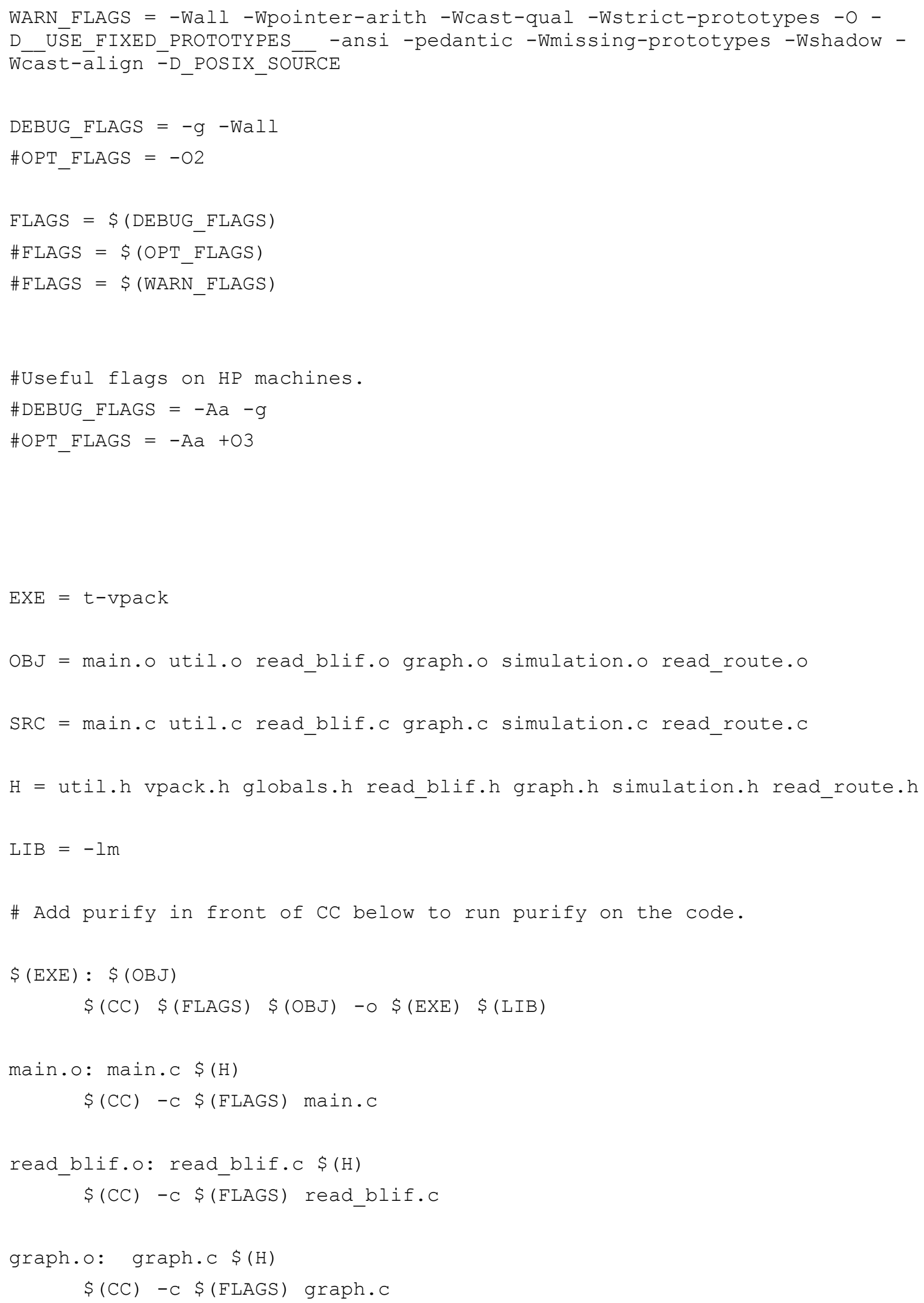




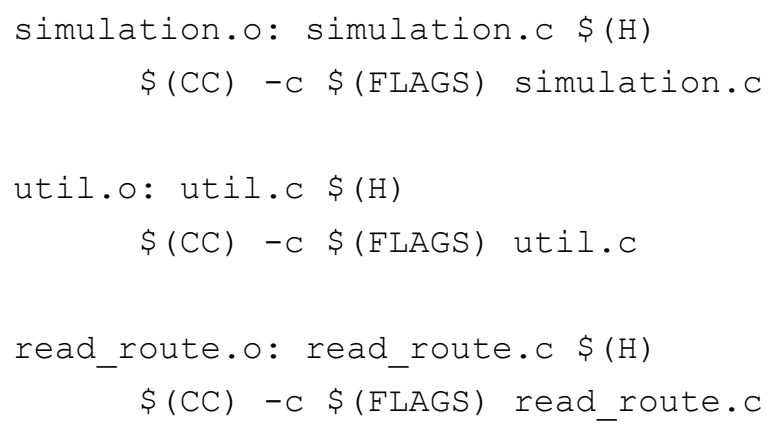




\section{Bibliography}

[1] J. Rose, A. E. Gamal and A. S. Vincentell, "Architecture of Field-Programmable Gate Arrays," Proceedings of the IEEE, vol. 81, no. 7, pp. 1013-1029, 1993.

[2] J. Fowers, G. Brown, P. Cooke and G. Stitt, "A Performance and Energy Comparison of FPGAs, GPUs, and Multicores for Sliding Window Applications," in Proceedings of the ACM/SIGDA international symposium on Field Programmable Gate Arrays, 2012.

[3] J. D. Owens, M. Houston, D. Luebke, S. Green, J. E. Stone and J. C. Phillips, "GPU Computing," Proceedings of the IEEE, vol. 96, no. 5, pp. 879-899, 2008.

[4] Technologies, Qualcomm, "Qualcomm Snapdragon 820 Processor," [Online]. Available: https://www.qualcomm.com/documents/snapdragon-820-processor-product-brief.

[5] J. Rose, "Hard vs. Soft: The Central Question of Pre-Fabricated Silicon," in Proceedings of the 34th International Symposium on Multiple-Valued Logic (ISMVL’04), 2004.

[6] I. Kuon and J. Rose, "Measuring the Gap Between FPGAs and ASICs," in IEEE Transactions on Computer-Aided Design of Integrated Circuits and Systems, 2007.

[7] A. Dehon, "Wordwidth, Instructions, Looping, and Virtualization The Role of Sharing in Absolute Energy Minimization," in FPGA '14 Proceedings of the 2014 ACM/SIGDA international symposium on Field-programmable gate arrays, 2014.

[8] N. Goulding-Hotta, J. Sampson, G. Venkatesh, S. Garcia, J. Auricchio, P.-C. Huang, M. Arora, S. Nath, V. Bhatt, J. Babb, S. Swanson and M. B. Taylor, "The GreenDroid Mobile Application Processor: An Architecture for Silicon's Dark Future," IEEE Micro, vol. 31, no. 2, pp. 86-95, 2011.

[9] Altera Corporation, "Standard Cell ASIC to FPGA Design Methodology and Guidelines," Altera Corporation, 2009. 
[10] A. Marquardt, V. Betz and J. Rose, "Using cluster-based logic blocks and timing-driven packing to improve FPGA speed and density," in FPGA '99 Proceedings of the 1999 ACM/SIGDA seventh international symposium on Field programmable gate arrays, 1999.

[11] V. Betz and J. Rose, "Cluster-based logic blocks for FPGAs: area-efficiency vs. input sharing and size," in IEEE 1997 Custom Integrated Circuits Conference, 1997.

[12] J. Rose, R. J. Francis, D. Lewis and P. Chow, "Architecture of Field Programmable Gate Arrays: The Effect of Logic Block Complexity on Area Efficiency," IEEE Journal of Soild State Circuits, vol. 25, no. 5, pp. 1217-1225, 1990.

[13] D. Chen, J. Cong and P. Pan, "FPGA Design Automation: A Survey," Foundations and Trends in Electronic Design Automation, vol. 1, no. 3, pp. 139-169, 2006.

[14] K. E. Murray, "Divide-and-Conquer Techniques for Large Scale FPGA Design," University of Toronto, 2015.

[15] A. Canis, J. Choi, M. Aldham, V. Zhang, A. Kammoona, J. Anderson, S. Brown and T. Czajkowski, "LegUp: high-level synthesis for FPGA-based processor/accelerator systems," in Proceedings of the 19th ACM/SIGDA international symposium on Field programmable gate arrays, 2011.

[16] Altera Corporation, "Implementing FPGA Design with the OpenCL Standard," 2012.

[17] J. Lamoureux and S. J. Wilton, "On the Interaction Between Power-Aware FPGA CAD Algorithms," in Proceedings of the 2003 IEEE/ACM international conference on Computeraided design, 2003.

[18] A. Marquardt, V. Betz and J. Rose, "Timing-Driven Placement for FPGAs," in Proceedings of the 2000 ACM/SIGDA eighth international symposium on Field programmable gate arrays, 2000.

[19] S. Kirkpatrick, C. D. Gelatt and M. P. Vecchi, "Optimization by Simulated Annealing," Science, vol. 220, no. 4598, pp. 671-680, 13 May 1983. 
[20] F. Rubin, "The Lee Path Connection Algorithm," IEEE Transactions on Computers, Vols. c-23, no. 9, pp. 907-914, 1974.

[21] S. M. Sait and H. Youssef, VLSI Physical Design Automation: Theory and Practice, World Scientific Publishing Co. Pte. Ltd., 1999.

[22] E. Bozorgzadeh, S. Ogrenci-Memik and M. Sarrafzadeh, "RPack: Routability-Driven packing for cluster-based FPGAs," in Proceedings of the 2001 Asia and South Pacific Design Automation Conference, 2001.

[23] A. Singh, G. PARTHASARATHY and M. MAREK-SADOWSKA, "Efficient Circuit Clustering for Area and Power Reduction in FPGAs," ACM Transactions on Design Automation of Electronic Systems (TODAES), vol. 7, no. 4, pp. 643-663, 2002.

[24] A. W. Burks, H. H. Goldstine and J. v. Neumann, "Preliminary Discussion of The Logical Design of an Electronic Computing Instrument," Princeton, 1946.

[25] M. D. Blasi, Computer Architecture, Addison Wesley, 1990.

[26] J. P. Hayes, Computer Architecture and Organization, McGraw-Hill, 1998.

[27] W. Wolf, Computers as Components, Morgan Kaufmann Publishers, 2008.

[28] I. Koren, Computer Arithmatic Algorithms, Universities Press, 2002.

[29] N. Weste and D. M. Harris, CMOS VLSI Design: A Circuits and Systems Perspective, Addison-Wesley, 2011.

[30] V. Betz and J. Rose, "VPR: A New Packing, Placement and Routing Tool for FPGA Research," in International Workshop on Field Programmable Logic, London, UK, 1997.

[31] B. University of California, "Berkeley logic interchange format (BLIF)," Tool Distribution, vol. 2, pp. 197-247, 1992.

[32] T. H. Cormen, C. E. Leiserson, R. L. Rivest and C. Stein, Introduction to Algorithms, The MIT Press, 2009. 
[33] A. V. Aho, M. S. Lam, R. Sethi and J. D. Ullman, Compilers: Principles, Techniques and Tools, Pearson: Addison-Wesley, 2007.

[34] V. Betz, J. Rose and A. Marquardt, Architecture and CAD for Deep-Submicron FPGAs, Kluwer Academic Publishers, 1999.

[35] F. F. Khan and A. Ye, "Measuring the Accuracy of Minimum Width Transistor Area in Estimating FPGA Layout Area," in IEEE 23rd Annual International Symposium on FieldProgrammable Custom Computing Machines, 2015.

[36] I. Kuon, R. Tessier and J. Rose, "FPGA Architecture: Survey and Challenges," Foundations and Trends in Electronic Design Automation, vol. 2, no. 2, pp. 135-253, 2008. 


\section{Glossary}

FPGA Field Programmable Gate Array

LUT Look up Table

GPU Graphics Processing Unit

DSP Digital Signal Processor

CPU Central Processing Unit

ASIC Application Specific Integrated Circuits

MCNC Microelectronics Centre of North Carolina

IC Integrated Circuit

NRE Non-Recurring Engineering

BRAM Block Random Access Memory

DFF D-Flip Flop

BLE Basic Logic Element

LB Logic Block

HDL Hardware Description Language

CAD Computer Aided Design

HLS High Level Synthesis

FA Full Adder

BLIF Berkeley Logic Interchange Format

PE Processing Element

VPR Versatile Place and Route

SOC System on Chip 